\title{
CALCULATIONS OF THE ELECTRON-DAMPING FORCE ON
}

\section{YOVING-EDGE DISLOCATIONS}

\author{
Tetsuo Hohri \\ Materials and Molecular Research Division, \\ Lawrence Berkeley Laboratory
}

and

Department of Materials Science and Mineral Engineering.

University of California. Berkeley, California 94720

NOTICE

FOATLWS OF THIS MEPOAT ARE ILLEGIBLE.

ABSTRACT

It has bean reproduced fram the best aveliable copy to permit the broadest possible availability.

Dynamic effect of a moving dislocation has been recognized as one of essential features of deformation behavior at very low temperatures. Damping mechanisms are the central problems in this fleld. Based on the free-electron-gas model, the electron-damping torce (riction iorce) on a moving-edge dislocation in a normal state is estimated. By applying classical Mackenzie-Sondheimer's procedures, the electrical resistivity caused by a moving dislocation is Arst estimated, and the damping force is calculated as a Joule-heat-energy dissipation. The calculated values are $3.63 \times 10^{-8}$,

This maniscript was printed from originals provided by the author. 
$7.62 \times 10^{-7}$ and $2.00 \times 10^{-6}\left[\mathrm{dyn}\right.$ sec $\left./ \mathrm{cm}^{-2}\right]$ for $\mathrm{Al}, \mathrm{Cu}$ and $\mathrm{Pb}$, respectively. These values show fairly good agreements as compared with experimental results. Also, numerical calculations are carried out to estimate magnetic eflects caused by a moving dislocation. The results are negative and any magnetic eflects are not expected.

In order to treat deformation behavior at very low temperatures, a unifleation of three important deformation problems is attempted and a fundamental equation is dorived. 


\section{ACKNOTLEBGEYHATS}

My sincere thanks to Professor J. W. Morris, Jr. for his guidance and support throughout the course of this work, and to Professors D. de Fontaine and Ole $H$. Hald for their reviewing this manuscript. Special thanks to Dr. Warren Garrison, Ir. for his reviewing this manuscript, and to Mr. Gianni Fior for his compuler assistence, as well as for their friendships.

I would like to acknowledge the staff and faculty in both the Hearst Mining Building and M.M.R.D. of the Lawrence Berkeley Laboratory, especially Mrs. Jeanne Shull and Gloria Pelatowski.

Thanks is due to Professor and Mrs. Leo Kanowitz for their continuous encouragement and enlightenment throughout my life here in United States.

Finally I would like to thank my family especially my parents and my wife, Fumiko, for their encouragement, support and everything.

This work was supported by the Director, Offlee of Energy Research. Office of Basic Energy Sciences, Materiais Sciences Division of the U. S. Department of Energy under contract No. DE-ACO3-76SF00098.

\section{DISCLAIMER}

This report was peepared as an account of work sponsored by an agency of the United States Government. Neither the United States Gowemment nor any agency thereof, nor any of their employees, makes any warranty, express or implied, or assumes any legal liability or responsibility for the accuracy, completeness, or usefulness of any information, apperatus, product, or process disclosed, or represents that its use would nor infringe privately owsed rights. Referenoe herein to any specific commercial product, prosess, or service by trads name, trademerk, manufacturer, or otherwise dees nor necessarily constitute or imply its endorsement, reconmendation, of favoring by the United States Government or any agency thereof. The views and opinions of authors expressed herein do not necessarily state or reflect thone of the United States Government or any agency thereol. 
TABLE OF CONTENTS

ABSTRACT

Chapter I.

INTRODUCTION,

1. General Characterization of Theoretical

Problems of Dislocation...................... I

2. Elementary Interaction Process................ 2

3. Damping Force........................ 5

4. Research Problem....................... 7

Chapter II. ELECTRODYNAMICAL FORMULATION OF AN

EDGE DISLOCATION MOTION...................... 15

1. Maxwell's Equations and Lorentz Transformation.... 15

2. Representation of a Moving Edge Dislocation Motion in Two Inertia Coordinate Systems........ 17

3. Establishnent of Maxwell's Equations for a Moving Edge Dislocation..................... 20

Chapter III. CALCULATION PROCEDURES...................... 22

1. Electrostatic Potential and Electron Density Around a Dislocation......................... 22

2. Establishnent of Boltzman Transport Equation ad Mackenzie-Sondheimer's Procedures........... 27

2-1. Establishnent of Boltzman Transport Equation..... 27

2-2. Flectrical Resistivity.................. 34

3. Friction Force........................... 39

4. Magnetic Induction......................4I

Chapter IV. RESULTS AND DISCUSSION.................... 45

1. Electron Density and Perturbation Potential...... 45

2. Born Approxination..................... 49

3. Boltzman Transport Equation and MackenzieSondheimer's Procedures...................... 50

4. Friction Force and Electrical Resistivity....... 51

5. Magnetic Induction and Magnetic Interaction....... 59

6. Possibility of the Extension to Many Body Problem (Collective Behavior of Dislocations) in Very Low Temperature Deformation............. 61

$6-1$. Sumino Hypothesis....................61 
6-2. Extension to Free Flight Thermally Activated Motion with the Aid of Dynamic Effect..........6.63

Chapter $v . \quad$ CONCLUSTONG............................. 70

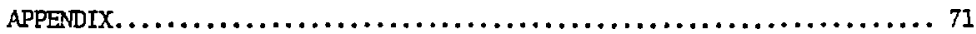

FIGURES. .......................................... 84 TABLES............................................. 107 REFERENCES........................................... 


\section{RMRODUCTON}

\section{1- General Characterization of Theoretical Problems of Dislocation Behavior}

Various properties of materials, optical, electrical, chemical, mechanical, etc., are more or less infuenced by dislocations. Especially, the mechanical properties of metals and alloys which are central concerns of physical metallurgy are largely determined by dislocation behavior. Although sigaificant progress in dislocation theory has been made, the basic understanding of physical properties of dislocations is still insuffeient to supply a useful guide lines for alloy design. A general characterization of the important probleins in dislocation theory based on the author's point of view is summarized below.

It is well known that the dislocation density of a well annealed metal is approximately $10^{6} \mathrm{~cm}^{-2}$ and that of a deformed metal is approximately $10^{12} \mathrm{~cm}^{-2}$ which is 250 times as long as our equator length. These tremendous amounts of dislocations are of ten observed to interact in a very complicated manner and to tangle one another. However complicated their configurations and interactions might be. since plastic deformation is a natural phenomena the group behavior of these dislocations must be strictly obeyed by certain physical principles or natural laws. The search for such a physical principle known as the "collective behavior of dislocations" or "multiple behavior of dislocations" is considered to be an extremely important probiem in a analysis of macroscopic mechanical behavior. Although the theory has been mostly devoted to single dislecation behavior no one can really understand and predict macroscopic crystal plasticity without the knowledge of the collective behavior of dislocations. A key factor of this problem is the development of a suitable thermodynamical treatment.

Apart trom the many body problems of dislocation theory mentioned 
above, the behavior of single dislocations is of fundamental importance. This problem can be split into two sub-categories.

One is the "elementary interaction process" which deals with interaction process between single dislocations and various types of obstacles such as solute atoms, 2nd phase, other dislocations and so on. This problem, the main concern of this thesis, is discussed in detail in the next section.

The other category is characterized as a statistical aspect of a dislocation behavior. Critical resolved shear stress (c.r.s.s.) can not be uniquely determined even if the elementary interaction process between a dislocation and a obstacle is well established. This is because c.r.s.s. is not a simple sum of each resistance force caused by obstacles but complicated function of the distributions, concentrations and strength spectrums etc. of obstacles which are scattered on a slip plane and of the line tension of a dislocation. Direct computer simulation techniques have been most successiully applied to this statistical problem and analytical calculations based on geometrical consideration or functional analysis were examined.

Attentions should be paid equally to these three categories in order to fully understand dislocation behavior and to develop a dislocation theory of power suffeient to provide realistic criteria lor the design of new alloys. This study is, howe ver, focussed on the elementary interaction process and the details of this problem are tatroduced in the next section.

\section{I-2. Blementary Interaction Process}

Since the first theoretical prediction of the existence of a dislocation line by Ewing et.al. in 1899 [1] most theoretical efforts have been concentrated on the problem of how to explain macrcscopically observed strength in terms of microscopic dislocation interactions with varinus types of obstacles. Typical 
examples are found in studies of solid solution hardening and dispersion hardening effects. These calculations are based on continuum elasticity theory and a static equilibrium confguration between a dislocation and a obstacle is assumed. Furthermore, rate processes have been described within a frame of absolute rate theory of Eyring.

However, with the progress ol low temperature measurement techniques in this fifteen years, various types of abnormal deformation behavior which can not be explained by simple extragoiation of high temperature deformation mechanisms have been reported. The sudden change of fow stress of a lead polycrystal due to the transition between the normal and superconducting states measured by Suzuki ex.al.[2] and Pustovalor et.al [3] in 1968, or the abrupt drop in the temperature dependence of the yield stress of $\mathrm{Cu}$ and its dilute alloys below $50^{\circ} \mathrm{K}$ tound by Kamata et.al.[4] are typical examples of those anomalies. Since, at present, it is still difficuit to extend the in-situ TEM technique to low temperatures and to observe a dislocation behavior directly, one should neither jump at the conclusion nor ascribe these anomalies to a single cause. But, by comparing the results of different experimental approaches, the dynamic interaction of dislocations with "microscopic obstacles" sush as phonons, electrons etc. has been recognized as most plausible source of these anomalies. Especially, in C.c.c. metals of which Peierls potential is relatively small. the dominant moda of dislocation motion is free light motion (spurt-like motion) and dislocation velocities of up to $10^{2 \sim 3} \mathrm{~cm} / \mathrm{sec}[5]$ have been measured in $\mathrm{Cu}$ even at room temperature. These facts might quite naturally iead one to the idea of a dyamic effect.

Mathematically the anomalies mentioned above can be re-explained in the lollowing way. According to the string model of a dislocation which was originally introduced by Koehler [6] the motion equation can be written as 


$$
m_{\alpha} \frac{\partial^{2} y}{\partial t^{2}}+B \frac{\partial y}{\partial t}+\Gamma \frac{\partial^{2} y}{\partial x^{2}}+\tau_{a f \rho^{b}} b=0
$$

where $m_{\text {rd }}$ is the mass of a dislocation per unit length, $B$ is a Jamping constent caused by electrons or phonons etc., $\Gamma$ is the line tension, $b$ is the magnitude of the Burgers vector, $(x, y)$ is the courdinate system on the active slip plane, $t$ is time and $\tau_{\text {off }}$ is the effective stress on the dislocation line. This effective stress is explicitly given by

$$
\tau_{\text {afs }}=\tau_{\text {app }}-\tau_{i}(z, y)
$$

where $\tau_{\text {opp }}$ is the applied stress and $\tau_{i}(x, y)$ is the internal stress caused by obstacles.

Therefore eq.1-1 is the force balance condition among inertia force, damping force, line tension and effective applied stress on a dislocation. In earlier treatments, the last two terms were suffeient enough to describe the static equilibrium condition, and primary interest was the functional form of the internal stress, $\tau_{1}(z, y)$, including its spatiei distribution, strength spectrum etc.. However, abnormal plasticity of low temperatures implies two additional dynamic terms, namely fnertia force and damping force. These additional terms will force one to reexamine the statistical problem mentioned in the former section and will not permit the rate process to be described by simple absolute rate theory of Eyring. Especially, the latter problem, how to combine thermally activated processes with lonetics, poses tremendous diffeulties and can not be resolved without returning the lundamental physics.

The main object of this study is the investigation of the physical origin of the "microscopic interaction", namely damping, and the estimation of the magnitude of this damping force. 


\section{1-3. Damping Force}

There are many damping mechanisms tor a dislocation. Among them, the following three mechanisms are important. They are phonon damping. electron damping and reradiation damping. Therefore the damping constant, $B$, in the eq. $1-1$ is rewritten as

$$
B=B_{\rho}+B_{0}+B_{\mathrm{r}}
$$

Phonon damping can be further divided into two mechanisms. One is phonon scattering of which the main process is the scatiering oi sionons by the dislocation strain field, and the other is phonon viscosity which involves the separation of the effective temperatures of different phonon modes produced by th. shear stress field of the moving dislocation and its relaxation to equilibrium. which was proposed by Mason [7]. Phonon damping has been revealed to be monotonically increasing with temperature, and in the case of very low temperatures where the lattice vibration is suppressed, phnnon damping is virtually negligible. Since this study is limited to very low temperatures deformation the phonon mechanism is not considered here.

For suffciently low temperatures, the remaining mechanisms are electron damping and reradiation. Although the reradiation of elastic waves of vibratiog dislocation is significant in insuiators and superconductors, this mechanism is less effective than electron damping for normal netals. And the main object of this study is confined to electron darning mecbanism.

As pointed out in the last section, the existence of the electron damping was Arst indicated in the tensile test of $\mathrm{Pb}$ crystal as the abnormal enhancement of plasticity due to the transition from the normal to superconducting states [2]. Creep and stress relaxation tests also conffmed the anomalies whish could be ascribed to the electron damping force $[8,9,10]$. These facts are ApL 
to give the impression that electron damping is assuciated wich only the normal-superconducting trinsition. However, effect is universal and is merely emphesized by the trarsition. Moreover, one should nctise that it is not an easy task to extract pure component of electron damping force from the measured stress change associated with the normal-siperconducting transition. For example, all the measurements were performed below the critical temperature and an external magnetic neld was applied to bring the sample back to the no:mal stat:?. The magc stic fleld orght infuence the dislocation behavior, either directly or indirectly through the cliange of et zctronic structure of the sarnple. In realiz, escent experiment of Galligan [11] impless this effect. Probably only a measurement under continuous cooling through the transition temperature could resolve this point. At any rate, without overcoming these experimental difficulties. the observed difference due to the normal-superconducting transition can not be fully ascribed to the electron damping fice. This point is discussed in chapter IV-4.

By the way, dislocation behavior in the supercondicting state is expected to be much diferent from that in the normal state. Anomalous strain rate sensitivity of fow stress measured in superconducting $\mathrm{Al}$ and $\mathrm{Al}-\mathrm{Mg}$ alloys is one of the typical examples [12], and this phenomenon is explained as the destruction of the Cooper pairs to create extra-quasi particles which is caused by dislocutions moving with greater relocity than certain critical level. Vartous other types of quantum natures are more or less expected in the deformation properties of this state, as well Then. as was calculated on a stralght dislocation by Hufman et.al. [13] and extended to loop dislocation by Bar'yakhtar et.al. [14]. it is certaniy of great importance to investigate damping mecharism ii- superconductors utilizing a rigorous quantum mecharical treatment. However, tho main scope of the present study is neither to investigate the dislocation 
behavior of superconductors nor to predict new phenomenon by pertorming rigorous treatment. This study is confoned to the electron damping mechanism in the normal state and to the discussion of possibility of the extension to many dislocations problem.

\section{F-4. Research Problem}

The previous theoretical studies of electron damping mechanism in the normal state are reviewed. The Arst theoretical calculation may be attributed to the classical viscosity theory of Mason [15, 16]. A dislocation is surrounded ay strain fleld that moves with the dislocation, and tree electrons in the solid exert a viscous effect on an ultrasonic wave propagated in the crystal with viscosity $\eta$. In other words a lattice vibration caused by dislocation motion can communicate energy to the tree electron gas by a viscous reaction (i.e. transter of momentum) and is damped by the viscosity of the gas. Accarding to the classical viscosity theory of gas, the viscosity, $\eta$, is given by .

$$
\eta=N m \bar{l} \frac{\vec{v}}{3}
$$

where $N$ is the number of particle, $m$ the mass, $\tau$ the mean free path, and $\vec{v}$ is the mean velocity. Mason applied the free electron gas model to the eq.1-4 and substituted the following particular quantities of free electron gas.

$$
\begin{aligned}
& \tau=\frac{\sigma m \bar{v}}{N \theta^{2}} \\
& \overline{v^{2}}=\frac{3}{5} \frac{\hbar^{2}}{m^{2}}\left(3 \pi^{2} N\right)^{\frac{2}{5}}
\end{aligned}
$$

where $\sigma$ is the electrical conductivity. And as a final form, be obtained

$$
\eta_{*}=\frac{9 \times 10^{11} \kappa^{2}\left(3 \pi^{2} N\right)^{\frac{2}{3}}}{5 \theta^{2} p}
$$


where $\rho$ is the electrical resistivity. By equating the energy dissipation associated witb the viscosity $\eta_{0}$ with the rate of change of the strain fleld as the dislocation moves through the crystal, the damping force and therefore the damping constant $B_{\text {e }}$ was calculated for the edge dislocation

$$
B_{0}=\frac{\frac{3}{4} b^{2} \eta_{0}}{6 \pi(1-\sigma)^{2} c_{0}^{2}}
$$

where $a_{0}$ is the cut-od radius. Although this treatment is simple and the physics is clear, a severe inconsistency should be noticed. As was criticized by Tittmann et.al. [17], the classical riscosity theory is valid only in the case of

$$
q \bar{l} \ll 1
$$

where $q$ is the typical Fourier component of the strain feld. Howerer, the typical value of $q$ is about the order of the reciprocal of atomic dimension and $\bar{\tau}$ is about $10^{-1} \mathrm{~cm}$ at liq. He temperature. Then $q \bar{l}$ is always far bigger than one for the electron-lattice system.

$$
q \Gamma \gg 1
$$

Therefore we should conclude that a proper electron damping can not be obtained by classical viscosity theorem.

Holstain [18] calculated the electron damping force based on perturbation metnod in the following way. The displacement feld, $a\left(c-\nabla_{D} t\right)$, around a moving dislocation with velocity $\nabla_{D}$ is expanded into Fourier series.

$$
\mathbf{u}\left(\mathbf{r}-\nabla_{D} t\right)=\sum_{\mathbf{q}} \mathbf{u}_{\mathbf{q}} \cdot \exp \left[i \mathbf{q}\left(\mathbf{r}-\nabla_{D} t\right)\right]+\text { Complexconjugate }
$$

Each $u_{q}$ is corresponding to lattice wave or lattice vibration of wave vector $q$ and gives rise to a deformation potential. The total deformation potantial. 
$V_{p}(r)$, is, then. given as the sum of the contributions of each $q q$ mode.

$$
V_{p}(\boldsymbol{r})=\sum_{q} i q \cdot u_{\mathbf{P}} \exp \left[i q \cdot\left(\mathbf{r}-\nabla_{D} t\right)\right]+\text { Complexconjugate }
$$

Due to this deformation potential conduction electrons are scattered accompanied with the absorption or emission of phonon, $\pi \mathrm{q} \cdot \mathbf{v}_{0}$ Holstein calculated the scattering probability matrix of this process by perturbation method and obtained the energy changing rate, $\frac{d W}{d t}$, in the following equation.

$$
\frac{d \boldsymbol{W}}{d t}=\sum_{\mathbf{k}} \sum_{q}\left(\pi \mathrm{q} \cdot \mathbf{v}_{D}\right)\left\{f\left(\mathbf{k}_{0}\right)-f(\mathbf{k})\right] \frac{2 \pi}{\pi} C^{2}\left|\mathbf{q} \cdot \mathbf{u}_{\mathbf{q}}\right|^{2} \cdot \delta\left[\frac{\pi}{m}\left[\frac{q^{2}}{2}+\mathbf{q} \cdot \mathbf{k}\right\}-\pi \mathrm{q} \cdot \mathbf{v}_{D}\right]
$$

where $\kappa \mathrm{q} \cdot v_{L}$ is the phonon energy and the remaining term expresses the transition probability of electron. $f(\mathbf{k})$ is the electron distribution luaction. By notleing the lact that

$$
\left|\mathbf{q} \cdot \mathbf{u}_{\mathbf{q}}\right|=|\Delta(\mathbf{q})|
$$

where $\Delta(q)$ is the Fourier transform of the dilatation, $\Delta(r)$. followed by several mathematical manipulations, the sum in the eq. 1-13 can be transtormed into integral and the following equation is obtained.

$$
\frac{d H}{d t}=\frac{L_{D}}{32 \pi} \frac{2 \pi}{\Gamma} \frac{C^{2}}{E^{2}} \frac{q_{m}}{k_{r}} k^{3} r^{2} B^{2} \pi^{2} x
$$

where $L_{D}$ is the dislocation line length. $q_{\mathrm{m}}$ is the maximum q-vector corresponding to the minimum possible wave lengtb and $x$ is approximately $1 / 4$. Holstein equated this anergy changing rate with power loss due to the friction force between electron and a moring dislocation of velocity $v_{D}$. 


$$
\frac{d \pi}{d t}=B L_{D} v D
$$

A flnal expression of the electron damping constant, $B$, is obtained in the following equation.

$$
B=\frac{3}{32} \frac{2 \pi}{\pi}\left(\frac{c}{E_{F}}\right)^{2}\left(\frac{q_{m}}{k_{F}}\right) k^{2} \xi \hbar^{2} N^{2} k
$$

On the contrary to the above perturbational approach based on quantum mechanics of Holstein, Hufman and Louat method [19, 20] described below is semi classical treatment. They calculated the electric current and electric fleld produced by deformation potential and moving dislocation by solving Maxrell's equation and Soltzman transport equation self-consistently. Then they obtained the friction force as a Obm power loss which is the product of the electric current and electric field. Later, this procedure wes stightly corrected by Brailsford[21] within a trame of the same physics. Their treatments are, however. straight application of the theory of ultrasonic attenuation which is traced back to the pioneer work of Cohen et.al.[22]. Essential procedures of Brailsford's are reviewed below.

The local lattice velocity, $u(r, t)$, is expanded into the Fourier series.

$$
u(r . t)=\sum_{q} u_{r} \exp \left[i q\left(r-\nabla_{D} t\right)\right]
$$

where $\nabla_{D}$ is the dislocation velocity and Fourier coeflleient $u_{\mathbf{n}}$ can be considered as the phonon of wave vector $q$ given by

$$
\mathbf{u}_{\mathbf{q}}=\sum_{\lambda}-i \mathbf{q} \cdot \mathbf{v}_{B} \mathbf{C}_{\boldsymbol{\lambda} \lambda} w_{q \lambda}
$$

Here $\lambda$ is an index specifying the normal made with polarization vector oq, and $w_{\nabla h}$ is the Fourier amplitude of the displacement associated with that mode. 
Each phonon (locgitudinal phonon) is associated with the dilatation teld which changes the electron density. The sell-consistent electric deld produced by the moving dislocation change the electron density as well. This prosess is described by the method of distribution function $f(r, 7, t)$, eq, $1-20$, and Boltzman Transport Equation, 1-21[23].

$$
\begin{aligned}
& f(\boldsymbol{r}, \mathbf{v}, t)=f^{0}(\nabla)+f_{1}(\boldsymbol{r}, \boldsymbol{v}, t) \\
& \frac{\partial f}{\partial t}+\nabla \frac{\partial f}{\partial \mathbf{r}}+\frac{\mathrm{eE}}{m} \frac{\partial f}{\partial \boldsymbol{r}}=-\frac{f-f^{0}}{\tau}
\end{aligned}
$$

where $f^{0}$ is the equilibrium electron distribution function, $f_{1}$ is the deviation from $f^{0}$ due to the electric feld $\boldsymbol{E}$, dilatation Aeld and collision with impurities of which relaxation time is $\tau$. Substitution of eq.1-20 into the eq. 1-21 followed by several mathematical manipulations yields

$$
\frac{\partial f_{1}}{\partial t}+\nabla \nabla_{x} f_{1}+\operatorname{eE} \nabla \frac{\partial f^{0}}{\partial \varepsilon}=-\frac{1}{\tau}\left[\left(m \nabla u+\frac{2}{3} \frac{n_{1}}{n_{0}} \varepsilon_{F}\right) \frac{\partial f^{0}}{\partial \varepsilon}+f_{1}\right)
$$

where $\pi_{0}$ is the equilibrium electron dengity and $n_{1}$ is the deviation from $n_{0}$ due to the dilatation field of the longitudinal phonon. The solution, $f_{1}$, of the eq. 122 is given by

$$
f_{1}=-\left[\frac{\operatorname{rev}\left[\mathrm{B}+\left(\frac{m u}{8 \tau}\right)\right]+\frac{2}{3} \varepsilon_{P}\left[\frac{n_{1}}{n_{0}}\right)}{1-i \omega \tau+i q \eta}\right] \frac{\partial f^{0}}{\partial t}
$$

where $\omega$ is

$$
\omega=\frac{q b}{\tau}-\frac{2 \pi n}{\tau}
$$

and $\mathrm{n}$ is an integer. Then, the electron current density, J, can be calculated by 


$$
J=\frac{2 e}{(2 \pi)^{5}} \int f_{1} \pi d k_{z} d k_{y} d k_{x}
$$

and the total electric current density $J_{T}$ is given by the sum of $J$ and positive lon current density, $-2 n u$.

$$
\mathbf{J}_{T}=\mathbf{J}-\mathbf{n e} \mathbf{u}
$$

On the other hand, electric field $\mathbf{E}$, can be correlated with $J$ by the following Maxwell's equation.

$$
\operatorname{div} \mathbf{B}=-4 \pi d i v d_{\tau}
$$

By solving eqs. 1-23, 1-25, 1-26 and 1-2? in a self-consistent manner. the electron current density $J$ and electric feld $\mathbf{B}$ can be obtained. And the product of $I$ and $\mathbf{E}$ immediately gives the ohm energy loss. According to more complete calculations of Brailsford, the energy absorption rate $P_{q}$ of a longitudinal phonon of $q$ is approximated to

$$
P_{1}=\left.\frac{\zeta_{0} m}{T}\left[1+\left(\frac{q}{q T_{P}}\right]^{2}\right]^{-2} \frac{1}{B} \pi q I_{0}\left|u_{v}\right|\right|^{2}
$$

where $q_{T_{r}}$ is the reciprocal of Thomas-Fermi screening length. Since the transversal phonon contribution is found to be negligibly small the total energy loss rate, $\mathrm{dW} / \mathrm{dt}$, is given by the sum of $P_{\mathbb{q}}$

$$
\frac{d W}{d t}=\sum_{t} P_{q}
$$

The anal step to obtain the damping constant $B_{0}$ is quite the same with that of Holstein's procedure, eq. 1-16. And the final expression for $B_{a}$ is given by

$$
B_{*}=\left(\frac{1-2 \nu_{P}}{1-\nu_{p}}\right)^{2} \frac{n_{0} m v_{p} b^{2} q_{d}}{96} \Phi\left(\frac{q_{D}}{q_{T_{F}}}\right)
$$


where

$$
\Phi(x)=\frac{1}{2}\left[\left(1+x^{2}\right)^{-1}+x^{-1} \tan ^{-1} x\right]
$$

and $q_{D}$ is the radius of Debye sphere.

Both the perturbational approach of Holstein and the self-consistent Boltzman Transport Equation method of Brailsford are very elegant method, however, the following two polnts are open to question.

In general, by introducing a $\mathrm{N}+1$ dimensional coordinate system which consists of $\mathrm{N}$ coordinate for atcmic displacements of each $\mathrm{N}$ atoms and the other coordinate for a potential energy of the system, the dislocation motion in a crystal of $\mathrm{N}$ atoms can be characterized as a trace along a one dimensionally extended saddle points configuration in the space. And the displacement of each atom caused by the motion of a dislocation can be given by the sum of a displacement corresponding to the saddle points and the deviation. The first component (saddle points) is so-called disiocation coordinate and the tatter one (dcriation) is regarded as phonon. Although, both Holstein and Brailstord treated a moving dislocation essentially as a wave packet of phonon, as discussed by Ninomiya [60], the dislocation coordinate given within an approximation of linear continuum elasileity theory does not always coincide with the saddle points but deviates. Then, in order to treat a dislocation motion as a phonon, one should be careful about not only allowable limit of the wavelength of phonon but also the width of the dislocation core which gives a meesure of the applicability of continuum approximation.

Secondly. the right-band side of eq. 1-21 is a scattering term but the mechanism is not speciffed clearly. If it is caused by impurities as was assumed by Cohen et.al. [22], the final result must be modifled by the concentration term. 
The theoretical calculation carried out in this study may not provide selfconsistent solution but demonstrates different approach to the electron damping problem.

This study proceeds in the following order. In chapter Il, general formulation based on the special theory of relativity is carried out. Beginning with a brief review of Lorentz transformation, two essential coordinate systems are introduced to characterize the dislocation motion, and Eaally Maxwell's equations appropriate to this problem is established. The chapter III is devoted to the calculation procedures, In the first section, calculations of electrastatic potential and electren density are performed by introducing Cettrell et.al's traditional work. The second section is the heart of this calculation and mathematically elegant Mackenzie and Sondtaimer's procedures are introduced to set up and to solve Boltzman Transport Equation. The Enal product is expressed as the electrical resistivity. The third section is the calculation of the friction force using the electric resistivity obtained in the former section. And in the last section. numerical calculations of magnetic induction are introduced. In the chapter IV, the results obtained in the chapter III are discussed. The Arst three siections discuss the applicavility of the Born approximation and the intrinsic insonsistency of Mackenzie-Sondheirner's method. The fourth section is the main discussion of the triction force. The method of the experimental analysis is examined, and in order to compare the calculated results more reliably, electric resistivity data are also introduced. The discrepancy is diseunsed by referring to Matthiesen's rule. In the afth section, the calculated magnetic induction value is shown to be negligible. The last section is unclosed work. The possibility of the extension of present study to many body problem ( collective behavior of dislocations) is discussed. 


\section{ELECTRODYNAYTCAL FORIULAITON OF AN EDGE DISLOCATION YOTON}

\section{II-1. Yaxwell's Equations and Lorentz Transformation}

Although dislocation velocities have never been observed to exceed $\sim 10^{\mathrm{J}}$ $\mathrm{cm} / \mathrm{sec}$ and any relativistic effects are hardly expected, the basic idea posed in the model, namely motion of charged particles associated with moving disl ccation. essentially belongs to electrodynamics. Therefore it is of basic interest to formulate this problem in a strict manner based on the special theory of relativity of Einstein.

The tensor expression of Marwell's Equations in Lorentz gauge are given by the following sets of equations [24].

$$
\begin{aligned}
& F_{\mu \nu}=\frac{\partial A_{\nu}}{\partial x_{\mu}}-\frac{\partial A_{\mu}}{\partial x_{\nu}} \\
& \mathscr{A}_{\mu \nu}=-\frac{1}{c \varepsilon_{0}} j_{\mu}
\end{aligned}
$$

and

$$
\frac{\partial A_{\mu}}{\partial x_{\mu}}=0
$$

where is the Lorentz invariant lour dimensional Laplacian more explicitly expressed as

$$
=\nabla^{2}-\frac{1}{\epsilon^{2}} \frac{\partial^{2}}{\partial t^{2}}
$$

$A_{\mu,} F_{\mu \nu}$ and $j_{\mu}$ are the 4-vector potential, leld-strength teasor, and charge -current 4-vector. respectively, and are concise totation of the following matrix. 


$$
\begin{aligned}
& \left(A_{\mu}\right)=(c \wedge, i \varphi) \\
& \left(F_{\mu \nu}\right)=\left[\begin{array}{cccc}
0 & c B_{x} & -c B_{y} & -i E_{x} \\
-c B_{x} & 0 & c B_{x} & -i E_{y} \\
c B_{y} & -c B_{x} & 0 & -i E_{x} \\
i E_{x} & i E_{y} & i E_{x} & 0
\end{array}\right] \\
& \left(j_{\mu}\right)=\left[\begin{array}{l}
i_{x} \\
i_{y} \\
i_{z} \\
i c \rho
\end{array}\right)
\end{aligned}
$$

where $a$ is the velocity of light, $A$ is the vector potential, $\varphi$, is the scalar potential, $E\left\{E_{x}, E_{y}, E_{x}\right)$ is the electronic field, $\mathbf{B}\left(B_{x}, B_{y}, B_{z}\right)$ is the magnetic flux density, $i\left(i_{2}, i_{y}, i_{j}\right)$ is the current density and $\rho$ is the charge density.

The Lcreatz transformation is characterized as an invariant transtormation which conserves the distance between two points in 4-dimensional Minkowski Space. The transformation law between the coorunates $x_{v}$ and $x_{j}^{j}(\nu, \mu ; 1,2,3,4)$ in two inertia systems, $K$ and $K$, are given by

$$
x_{\mu}^{\prime}=a_{\mu \nu}
$$

$x_{\mu}$ consists of three dimensional space coordinates, $x, y, z$ and one dimensional tume coordinate, $t$.

$$
\left[\begin{array}{l}
x_{1} \\
x_{2} \\
x_{3} \\
x_{4}
\end{array}\right]=\left[\begin{array}{l}
x \\
y \\
z \\
i c t
\end{array}\right]
$$

In the special case where system $K^{\prime}$ has its coordinate axes parallel to those of $K$ and is moving with a constant relative relocity $v$ in the $x$ direction. the transtormation matrix $a_{m}$ in the eq. $2-8$ can be simplifled and is given by the tollowing matrix. 


$$
\left(\alpha_{\mu \nu}\right)=\left[\begin{array}{cccc}
\frac{1}{\sqrt{1-\beta^{2}}} & 0 & 0 & \frac{i \beta^{*}}{\sqrt{1-\beta^{*}}} \\
0 & 1 & 0 & 0 \\
0 & 0 & 1 & 0 \\
\frac{-i \beta^{*}}{\sqrt{1-\beta^{\sigma^{2}}}} & 0 & 0 & \frac{1}{\sqrt{1-\beta^{2}}}
\end{array}\right]
$$

where

$$
\beta^{*}=\frac{v}{c}
$$

Corariance of the Maxmell's equations with respect to Lorentz transformation between two inertia system is an important consequence of the theory of relativity, and 4-vector potentials, $A_{\nu}$ and $A_{\mu}^{\prime}$, defined in the two systems are related by the following transformation law with the same transformation matrix 2-10.

$$
A_{\mu}^{\prime}=\alpha_{\mu \nu} A_{\nu}
$$

II-2. Representation of a Moving Edge Dislocation in Two Inertia Coondinate Syslems

Physical phenomena associated with a dislocation moving in a positive $x$ direction with constant velocity $v$ can be described in various coordinate systems. Among them, the following two orthogonal coordinate systems, $K$ and $K$, are of specifle interest in this study.

In the K-system, the coordinate is fxed to the lattice and the dislocation moves, in the positive $I$ direction with a constant velocity. $\nabla$, with respect to this lattice. On the other hand, in the $K$ '-system whose origin is fixed on the core of the dislocation, the coordinate rnoves with the dislocation. Ther ofore, in the $\mathrm{K}^{\prime}$-system. media surrounding ihe dislocation moves in the negative $x^{\prime}$ direction with velocity $v$. The conffgurational relation among the $K$-system, $\kappa$-system and the disiocation is shown in Fìg. 1. 
Due to the introduction of an edge dislocation, a displacement feld is induced. In the $K$-system, components of the displacement field, $u_{3}^{K}, v_{i}^{K}, u_{\pi}^{K}$ in the $x, y, 2$ direction, respectively, are given by [25]

$$
\begin{aligned}
& u_{*}^{\pi}=\frac{b}{2 \pi}\left(\frac{2 v_{s}^{2}}{v^{2}}\right]\left\{\tan ^{-1} \frac{\gamma y}{x-u t}-a^{2} \tan ^{-1} \frac{\beta y}{x-v t}\right\}
\end{aligned}
$$

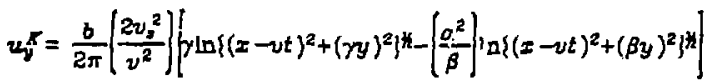

and

$$
u_{*}^{x}=0
$$

where superscript. $K$ stands for the $K$-system, $v$, is the transvers? velocity of sound, and $\alpha . \beta . \gamma$ are given by the following relations with longitudinal sound velocity $v_{l}$.

$$
\begin{aligned}
& a=\left[1-\left(\frac{v^{2}}{2 v_{z}^{2}}\right)\right]^{H} \\
& \beta=\left[1-\left(\frac{v^{2}}{v_{z}^{2}}\right)\right]^{H} . \\
& \gamma=\left\{1-\left(\frac{v^{2}}{v_{1}^{2}}\right)\right\}^{x}
\end{aligned}
$$

and superscript $K$ stards for the $K$-system.

In the $K$-system, however, these components are moditled according to the Lorentz transformation in the following way.

$$
u_{z}^{x}=\frac{b}{2 \pi}\left[\frac{2 v_{z}^{2}}{v^{2}}\right]\left\{\tan ^{-1} \frac{x y^{\prime}}{\delta x^{\prime}}-\alpha^{2} \tan ^{-1} \frac{\delta y^{\prime}}{\delta x^{\prime}}\right\}
$$




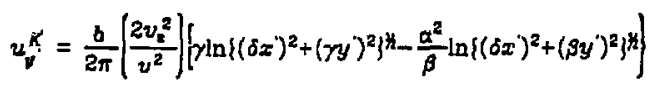

and

$$
u_{x}^{x}=0
$$

where

$$
\delta=\sqrt{1-\left(\beta^{\prime}\right)^{2}}
$$

and the next relations derived Irom eqgs. 2-B and 2-10 were employed.

$$
\left(\begin{array}{c}
z \\
y \\
z \\
i c t
\end{array}\right)=\left(\begin{array}{c}
\frac{1}{\delta}\left(x^{\prime}+u t^{\prime}\right) \\
y \\
z \\
\frac{i}{\delta}\left(\beta^{*} x^{\prime}+c t^{\prime}\right)
\end{array}\right)
$$

The dilatation fleld, $\Delta_{K}$, is calculated from the components of the displacement fleld obtained above.

$$
\begin{aligned}
\Delta_{X} & =\frac{\partial u_{i}^{K}}{\partial x_{i}^{\prime}} \\
& =\frac{b}{2 \pi}\left(\frac{2 u_{s}^{2}}{v^{2}}\right)\left\{\frac{\gamma y\left(\gamma^{2}-\delta\right)}{\left(\delta x^{\prime}\right)^{2}+\left(\gamma y^{\prime}\right)^{2}}+\frac{\alpha^{2} \beta(\delta-1) y^{\cdot}}{(\delta)^{2}+\beta y^{2}}\right\}
\end{aligned}
$$

where $\left(x_{1}, x_{2}, x_{j}\right)=\left(x^{\prime}, y^{\prime}, z^{\prime}\right)$. and $\left(u_{j}, u_{2}, u_{j}\right)=\left\{u_{x}, u_{\psi}, u_{x}\right)$. Because of this dilatation fleld the positive charge deasity (positive ionic density). $\rho_{K}^{+}$becomes heterogeneous and is given by

$$
\begin{aligned}
\rho_{K}^{+} & =\rho_{\mathbf{O}_{K}^{+}}^{+}\left(1+\Delta_{K}\right)^{-1} \\
& =\frac{\rho_{0}^{+} K}{\delta}\left(1+\Delta_{K}\right)^{-1}
\end{aligned}
$$


where $\rho_{a}^{+} K$ and $\rho_{0}^{+} k$ are uniform positive charge densities of the dislocation free crystal represented in $K$ - and $K^{\prime}$-system, respectively, and the modiacation trom the arst equation to the second cne is a direct consequence of the Lorentz contraction. It should be noliced that the oxplicit time term can be nuglected in the $K$-system representation eq. $2-24$, while not in the $K$-system formulation.

\section{I-3 Rstablishment of Yarwell's Rquations for a Yowing Edge vislocation}

Further discussions are cieveloped in the $K^{\prime}$-system instead of K-systein. becūuse, as was recognized in the last section. the explicit time terca disappears in the dilatation, eq. 2-24, and therefore the $K^{\prime}$-system is eesier to handle mathematicaliy. If need be, the covariant property of Maxwell's equations, eq. 2-12, allow the solution to be easily transformed into that of $\mathrm{K}$-system. Main interest is confined to the steady state, theretore the 4-dimensional Laplacian. can be simplified to the 3-dimensional Laplacian, $\nabla^{2}$, and Maxwell's equation can be rewritten as

$$
\nabla^{2}() A_{j}=-\frac{1}{c \varepsilon_{0}} j_{j}^{\prime}
$$

Here, by maknng use of the results obtained in the last section, components of the charge-current 4-vector $j_{\mu}^{\prime}$ are given by

$$
\left(j_{\mu}^{\prime}\right)=\left(\begin{array}{c}
-\frac{\rho_{0}^{+} \delta}{\delta} \\
0 \\
0 \\
\left.i c i \rho_{K}^{-}-\rho_{\alpha K}^{+}\left(1+\Delta_{K}\right)^{-1}\right\}
\end{array}\right)
$$

where $\rho_{\vec{A}}$ is the unknown electron density which plays an essential role in this study. Suostitution of eqs. 2-5 and eq. 2-27 inio eq. 2-26 establishes more explicit form of Maxmell's equations in the following way. 
21

$$
\nabla^{2}\left({ }^{\prime}\right) A_{x^{\prime}}=\frac{1}{\sigma^{2} \varepsilon_{0}}\left(\frac{\rho_{0}^{+} K}{\delta} \nu\right)
$$$$
\nabla^{2}(') A_{y^{\prime}}=0
$$

$$
\left.\nabla^{2}()^{\prime}\right) A_{x}=0
$$

$$
\nabla^{2}\left({ }^{\prime}\right) \varphi=-\frac{1}{\varepsilon_{0}}\left\{\rho_{K}^{-}-\rho_{0 K^{*}}^{+}\left(1+\Delta_{K}\right)^{-1}\right\}
$$

(2-28)

(2-29)

(2-31) 


\section{CALCULATION PFOCEDURES}

\section{III-1. Mectrostatic Potential and Electron Density Around a Dislocation}

A rigoraus treatment based on special theory of relativity was demonstrated in the last chapter. But as pizinted out previously; dislocation velocities are less than $m 10^{5} \mathrm{~cm} / \mathrm{sec}$ which is negligible as compared to the speed of light. Therefore, $\beta^{*}$ in the eq.2-11 and $\delta$ in the eq.2-22 can be put to 0 and 1 , respectively. This operation mathertatically corresponds to the reduction of the Lorentz transformation, eq. 2-23, to the following Galilei transformation

$$
\left[\begin{array}{l}
z \\
y \\
z
\end{array}\right]=\left(\begin{array}{c}
x^{\prime}+y t^{\prime} \\
y \\
z \\
t^{\prime}
\end{array}\right)
$$

and physically means that every phenomena is observed at the same time in both $K$ - and $K^{\prime}$ - system ln particular. this study of dislocation motion can be reduced to a treatment within a trame of electrostatics, and the first task is to obtain the electrostatic potential and electron density around a moving edge dislocation by focusing on the last equation 2-31 of Maxwell's equation.

The dilatation field around a dislocation causes the Fermi energy to change from place to place. Physical origins of this change are two-fold: change of the kinetic energy of eiectrons due to the change of wave length and change of ground level energy. both caused by lattice dilatation. However, in the equilibrium state the electrochemical potential should be a constant through the whole crystal, therelore electron redistribution takes place so that the electrostatic potential produced by the redistributed electrons compensates the Fermi energy change. This mecharism was originally developed by Cottrell et.al.[26] in order to estimate the elect;ic interaction energy between a 
staciz edge dislocation and a solute atom of diffarant valence.

Yathematically, these mechanisms are described in the tolloring equations. The Fermi energy around an edge dislocation is given by

$$
\begin{aligned}
\varepsilon_{P}(r) & =\varepsilon_{\dot{q}}+\frac{\hbar^{2}}{2 m} k_{\dot{p}}^{2} \\
& \cong \varepsilon_{\dot{q}}+\varepsilon_{1} \Delta+\frac{n^{2}}{2 m} k_{p} p_{1}(1+\Delta)^{-\frac{2}{3}}
\end{aligned}
$$

where $\varepsilon_{\varepsilon}$ and $\varepsilon_{G}$ are ground level energies of perfect and distorted crystals, $k_{F}$ and $k_{j}$ are the Fermi vectors of those crystals, respectively. In the above equations a Taylor expansion with respect to dilatation, $\Delta$. is carried oul on ground level energy. $\varepsilon_{G}$, and the orst two terms are considered. $\varepsilon_{1}$ could be found by the following fact; the equilibrium lattice parameter is determined by the condition that the derivative of the total energy of the conduction electrons with respect to $\Delta$ should ranish at $\Delta=0$. Thus.

$$
c_{1}=\frac{2}{5} \frac{n^{2} k F}{2 m}
$$

On the other hand, the condition of constant electrochemical potential is given by

$$
\begin{aligned}
\mu(\mathbf{r}) & =\varepsilon_{\beta}^{p} \\
& =\varepsilon_{P}(r)-8 \varphi(r)
\end{aligned}
$$

where $\mu(x)$ is the electrochemical potential at $r\left(x^{\prime}, y^{\prime}, z^{\prime}\right)$ which is equivalent to the Fermi energy of a perfect crystal (or Fermi energy at the region infinitely tar away from a disiocation) and $\varphi(r)$ is the electrostatic potential after the electron redistribution. Although. trom eq.3-2, the change of the Fermi energy, 
$\delta \varepsilon_{P}(\boldsymbol{r})$, can be given by

$$
\begin{aligned}
\delta \varepsilon_{r}(\mathbf{r}) & =\varepsilon_{r}(\mathbf{r})-\varepsilon \xi \\
& =-\frac{4}{15} \frac{\pi^{2}}{2 m} k \beta \Delta(\boldsymbol{r})
\end{aligned}
$$

and this net amount was implied to be compensated by electron redistribution in. the Cottrell's treatment, because of the electric field produced by positive ions, $\delta \varepsilon_{P}(r)$ can not be directly equated to $\phi(r)$ in eq. 3-4. Proper solution should be obtained by the simultaneous and self-consistent solutions of equations $2-31$ and $3-4$.

In order to proceed to actual computations the Thomas-Fermi approximation is performed on $\varepsilon_{F}(\mathbf{r})$ and eq. $3-4$ is rewritten in terms of electron density. $\rho_{\bar{K}}(r)$, in the next equation

$$
\rho_{\bar{X}}(r)=p_{\sigma K}^{-}\left(1-\frac{2}{5} \Delta+\frac{\varepsilon_{\varphi}}{\varepsilon_{\text {in }}^{0}}\right)^{\frac{3}{2}}
$$

where $\varepsilon_{\text {ind }}^{0}$ is the kinetic energy of electron in a perfect crystal and is given by

$$
\varepsilon_{m}^{0}=\frac{n^{2} k \beta}{2 m}
$$

Here. the simultaneous equations which give the equilibrium electron density and electrostatic potential around an edge dislocation are set up in the following way

$$
\begin{aligned}
& \rho^{-}=\rho_{0}^{-}\left\{1-\frac{3}{5} \Delta+\frac{3}{2} \frac{\varphi \varphi}{\varepsilon_{\operatorname{lin}}^{0}}\right\}\left(\text { provided that }-\frac{2}{5} \Delta+\frac{a \varphi}{\varepsilon_{\ln }^{0}} \ll 1\right) \\
& \nabla^{2} \varphi=-\frac{1}{\varepsilon_{0}}\left\{\rho^{-}-\rho_{0}^{-}(1+\Delta)^{-1}\right\}
\end{aligned}
$$


and

$$
\rho_{0}^{+}=\rho_{0}^{-}=\rho_{0}
$$

where the subscript $\mathrm{K}^{\prime}$ is neglected in order to avoid unnecessary complications.

Since it is quite tedious to demonstrate whole calculations of these simultaneous equations essential steps of the procedures are briefly summarized below.

Substitution of eq. 3-8 into eq. 3-9 yields the following partial differential equation (P.D.E) in a infinite two dimensional space.

$$
\left(\nabla^{2}-a\right) \varphi(\mathbf{r})=D \Delta(\mathbf{r})
$$

$$
\varphi \in \mathbf{R}_{2} \text { and } \varphi \rightarrow 0 \text { at }|\mathbf{r}| \rightarrow \infty
$$

where $a$ and $D$ are given by

$$
a=\frac{6 \pi e^{2} \rho_{0}}{\lim _{\sin }^{\mathrm{D}}}
$$

and

$$
D=\frac{8}{5} \pi e \rho_{0}
$$

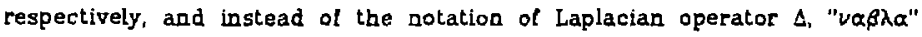
operator, $\nabla^{2}$, is used. The corresponding fundamental equation of P.D.E. 3-11 is given by

$$
\left(\nabla^{2}-a\right) g_{2}(r\{\xi\}=-\delta(r-\xi)
$$

and the Green's function is explicitly given by [27] 


$$
\epsilon_{2}(\mathbf{r} \mid \xi)=\frac{1}{2 \pi} K_{0}(\sqrt{a}|\mathbf{r}-\xi|)
$$

where $K_{0}$ is the 0 -th order modiffed Bessel function of the 2 nd kind. Therefore, with the aid of the Green's function the solution of P.D.E., 3-11 is obtained as

$$
\begin{aligned}
\varphi(\boldsymbol{x}) & =-\iint_{a}(\mathbf{r} \mid \xi) D \Delta(\xi) d \xi \\
& =-\frac{D}{a}\left(\Delta(\mathbf{r})-2 C \sqrt{a} K_{1}(\sqrt{a}|\mathbf{r}|) \frac{\sin \gamma}{\gamma+1}+\iint_{z_{2}}(\mathbf{r} \mid \xi) \nabla^{2} \Delta d \xi\right) \\
C & =\left\{-\frac{b}{2} \pi \mid 2\left(\frac{v_{z}}{u_{l}}\right)^{2}\right.
\end{aligned}
$$

where $K_{1}$ is the 1 st order modiffed Bessel lunction of the 2nd kind, $\gamma$ was given in the eq. $2-18$ and $v$ is measured anticlockwise from positive $x^{\prime}$ axis. in the eq. 3-16, the reduction of the upper integral into the lower equation is quite hard and despite of the persistent attacks with various mathematical techniques $\phi(r)$ could not be fully expressed by well detined functions. In order to evaluate the contribution of the 3rd term the integration was numerically performed tor various physically meaningful values of $a$ and $\gamma$, and the results were compared with 1st and Znd term. Several results are shown in Figs. 2, 3 and 4. One can see that the 3rd term is negligibly strall and can be safely neglected in the eq. 3-16. Therefore the fanal form of alectrostatic poteatial $\varphi(x)$ is vell approximated to

$$
\varphi(r)=-\frac{p}{a}\left|\Delta(\mathbf{r})-2 \sqrt{a} c \kappa_{1}(\sqrt{a}|\mathbf{r}|) \frac{\text { sind }}{\gamma+1}\right|
$$

afd substitution of the eq. 3-18 into eq. 3-8 yields

$$
p^{-}(r)=\rho_{0}\left\{1-\Delta+\frac{4}{5} C \sqrt{a} K_{1}(\sqrt{a}|r|) \frac{\sin \theta}{\gamma+1}\right\}
$$


These numerical processes are discussed in the section $N$ - 1 again but it is noticeable that in the case of a static dislocation the 3rd term in the eq. $3-16$ automatically vanishes and $\varphi(\mathbf{r})$ is strictly expressed as

$$
\phi(r)=-\frac{4}{15} \frac{1}{8} \frac{r^{2} k \xi}{2 m}\left(\Delta(r)-C \sqrt{a} K_{1}(\sqrt{a}|r|) \sin \vartheta\right)
$$

which includes extra screening contribution, the 2nd term, as compared with $\delta \varepsilon_{F}(r)$ of the eq. 3-5.

III-2. Bstablishment of Boltaman Transport Bquation and MackenaieSondheimer's Procedures

Various transport phenomena in a crystal can be well described by means of Boltzman Transport Equation (B.T.E.). Since the general leatures of this equation are discussed in detail in many articles. The essential points of this equation are briefly reviewed in this section and the B.T.E appropriate for this particular dislocation problem is established. In order to obtain the electrical resistivity by solving the B.T.E. analytically, classical Mackenzie-Sondheimer's procedures are introduced.

\section{III-2-1. Establishment of Boltrman Transport Equation}

Let $f(k, r, t)$ be the electron distribution lunction in a phase space. Under the given circumstances which are specified in the B.T.E., the probability of finding the electron of state $\mathbf{k}$ (momentum), at time $t$, in the space $r$. is described by this function. And this function as the solution of the B.T.E. plays the essential role in the study of transport phenomena. In the steady state the distribution function should satisfy the following condition.

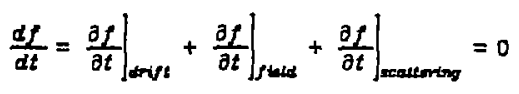


The drift and field terms are more explicitly given by

$$
\left.\frac{\partial f}{\partial t}\right|_{\text {drift }}=-\nabla_{\mathbf{r}} \cdot \nabla_{\mathrm{r}} f
$$

and

$$
\left.\frac{\partial f}{\partial t}\right]_{\text {flods }}=-\frac{e}{\pi}\left[E+\frac{1}{c} \eta_{k} \times H\right] \cdot \nabla_{k f}
$$

Where $\nabla_{z}$ and $\nabla_{k}$ are the vector differential operators in a phase space with respect to space coordinate $r$ and momentum coordinate $\mathbf{k}_{\text {, respectively; }} \mathbf{z}_{\mathbf{k}}$ is the group relocity of electron; $\boldsymbol{Z}$ is the external electric fleld and $H$ is the external magnetic field. The scattering tern is discussed later in detail. In the equilibrium state, the distribution function is the familiar Fermi-Dirac distribution function, $f^{\circ}(k)$, given by

$$
f^{\circ}(x)=\frac{1}{\exp \{(\varepsilon(k)-\zeta) / k T\}+1}
$$

where $\varepsilon$ is the energy of electron and $\xi$ is the electrochemical potential. By following Ziman [23], the steady state distribution function, $f(\mathbf{k}, \mathbf{r})$, is assumed not to depart very far from equilibrium distribution function, $f^{\circ}$, and the function $G(\mathbf{k}, \mathbf{r})$ is assigned to describe this small deviation.

$$
G(\mathbf{k}, \mathbf{r})=f(\mathbf{k}, \mathbf{r})-f^{\circ}(\mathbf{k})
$$

Subgtitution of eqs. 3-22 3-25 into the eq. 3-21 yields

$$
\begin{aligned}
& {\left[-\frac{\partial f^{0}}{\partial \varepsilon}\right) \nabla_{k}\left(-\frac{(\varepsilon(\xi)-\zeta)}{T} \nabla_{r} T+\alpha\left(E-\frac{1}{q} \nabla_{R} \zeta\right)\right]} \\
& \left.=-\frac{\partial f}{\partial t}\right]_{\text {rawering }}+\nabla_{2} \cdot \nabla_{Y} G+\frac{e}{\hbar}\left(\nabla_{Y} \times H\right) \cdot \nabla_{Y} G
\end{aligned}
$$


Interest here, bowever, is restricted to the case of homogeneous spatial temperature distribution. $T$, with the absence of magnetic fleld. $H$, therefore, above eq. $3-26$ is greatly simplified to

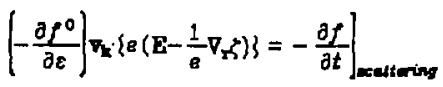

Moreover, as was demonstrated ir the last section, the spatial heterogeneity of electrochemical potential due to the introduction of a dislocation is canceled out by electron redistribution; therefore, eq.3-27 can be simplifled further.

$$
\left.\left(-\frac{\partial f^{0}}{\partial \varepsilon}\right) \nabla_{\mathbf{2}} \cdot \mathrm{E}=-\frac{\partial f}{\partial t}\right]_{\text {cottoring }}
$$

Here the scattering term in the eq.3-21 is discussed. In this study, two seattering mechanisms are assumed. One is thermal sattering and the other is disiocation scattering which is the particular feature of this model.

$$
\left.\frac{\partial f}{\partial t}\right]_{\text {socationing }}=\left.\frac{\partial f}{\partial t}\right|_{\text {them }}+\left.\frac{\partial f}{\partial t}\right|_{\text {ditlos. }}
$$

The actual mechanism of the thermal scattering is complicated, but without going into the detail this effect can be expressed by

$$
\left.\frac{\partial f}{\partial t}\right|_{\text {ANm }}=-\frac{f(k)-f^{0}}{\tau}
$$

where $T$ is the relaxation time and all the complicated physical mechanisms of thermal scattering are enclosed into tis "parameter". On the other hand, the mecbenism of dislocation scattering is well described in the following equation.

$$
\left.\frac{\partial f}{\partial t}\right|_{d i d \mid d x .}=C_{0}\left\{\iint_{\mathbf{k}} \int f\left(\mathbf{k}^{\prime}\right)(1-f(\mathbf{k})) Q(\mathbf{k}, \mathbf{k}) d \mathbf{k}^{\prime}-\iint_{\mathbf{k}} \int f(\mathbf{k})(1-f(\mathbf{k})) Q(\mathbf{k}, \mathbf{k}) d \mathbf{k}\right\}
$$


where $C_{0}$ is a constant and $Q(\mathbf{k}, \mathbf{k})$ is the transition probability from $\mathbf{k}$-state to k-state per unit time. Therefore, the first integral describes the incorning rate to $k$-state from all other states, the second integral describes the outgoing rate from $\mathbf{k}$-state to other states and hence the whole right hand side means the net change of k-state. Since scattering causes merely the change of state, only $k$ or $\mathbf{k}$ is explicitly written in the arguments of distribution function $f$. The physical mechanism of the dislocation scattering is directly reflected in the transition probability, $Q(\mathbf{K}, \mathbf{K})$, which is given by (APPENDLX A)

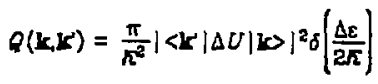

where Dirac's bra-ket notation is used fo: the matrix element and $|\mathbf{k}\rangle$ describes the iree electron wave function. (ket is the complex conjugate.)

$$
10 \sim g^{1 k r}
$$

The argument of the delta lunction appearing in the eq. 3-32 is the simple diference in energy between $\mathbf{k}$ and $\boldsymbol{k}$ states and is given by

$$
\Delta \varepsilon=\varepsilon(\mathbf{k})-\varepsilon(\mathbf{k})
$$

Then any inelastic scattaring processes are neglected in this study. As shown in APPENDIX A, this transition probability is derived within a trame of Born approximation [28] as the elastic iree electron scattering process due to the presence of perturbation (scattering) feld, $\Delta U$. As the perturbation potential, $\Delta U$. of an edge dislocation, the electrostatic potential feld derived in the last section is employed.

$$
\Delta U(\mathbf{r})=-8 \varphi(\mathbf{r})
$$


And the substitution of eqs. 3-29 3-32 and 3-35 into the eq. 3-28 immediately yields the B.T.E. for this study in the following torm.

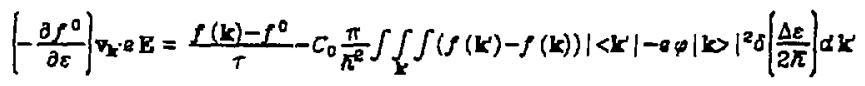

where the reduction of the integrands in the eq.3-31 to the eq.3-36 is based on the principle of microscopic reversibility [29]. The construction of the B.T.E. is, in principle, completed by the above equation 3-36 and the remaining task is to solve this integral equation. Although the appearance of the eq. 3-36 is complicated. mathematical techniques make it possible to reduce the triple integral to a single integral. The details of the whole procedure are quite tedious, and only the essential points are described here.

The key role is played by $\delta$-tunctions in the integrand. The first reduction from triple integral to double integral is rather a conventional technique often used in solid state physics [30]. The rolume element in the $\mathbf{K}$-space, $d \boldsymbol{K}$, is split Into the product of two cerms $d S_{\mathbf{K}}$ and $d K_{L}$.

$$
d \boldsymbol{k}=\boldsymbol{u} \mathbf{S}_{\mathbf{x}} \cdot d K_{t}
$$

where $d S_{\mathbf{Y}}$ is the surface element of the equi-energy surface in the $\mathbf{k}$-space and $d K_{+}$is the normal element to this surface, which satisfies the following condition

$$
\begin{aligned}
d K_{+} & =\frac{d \varepsilon(\mathbf{k})}{\left|\nabla_{\mathbf{k}} \varepsilon(\mathbf{k})\right|} \\
& =\frac{d \varepsilon(\mathbf{k})}{\left|\sqrt{\nabla_{\mathbf{k}}}\right|}
\end{aligned}
$$

here, from the frst line to the second line. the deffnition of the group velocity. 
7. of electron is applied. By substituting the eqs. 3-37 and 3-38 into the eq. 336 followed by the operation of the $\delta$-function, $\delta\left(\frac{\Delta \varepsilon}{2 \delta}\right)$, the volume integral is reduced to a surface integral over the equi-energy surface $S_{\mathrm{e}(\mathrm{k})}$.

$$
\begin{aligned}
& \int\left\{\int ( f ( \mathbf { k } ^ { \prime } ) - f ( \mathbf { k } ) \rangle \left\langle\mathbf{k}^{\prime}|-g \varphi| \mathbf{k}>\left.\right|^{2 \cdot} \cdot \dot{\sigma}^{\prime}\left[\frac{\Delta \varepsilon}{2 \pi}\right] d \mathbf{k}^{\prime}\right.\right.
\end{aligned}
$$

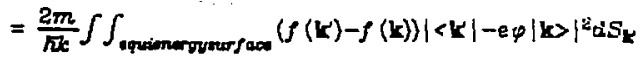

The coeflicient term of the right hand side originates from botb the denominator of the eq. 3-38 and the argument in the $\delta$-lunctioc, but is written in terms of k-vector instead of velocity. v. which are related to each other by the momeatum equation of the free electron.

$$
m \nabla=n \mathbf{r}
$$

The second reduction, from double integral to single integral, requires more tedious variable transformations, but the essential point is arain ascribed to the delta function as is mentioned below. One will notice that the scattering probability matrix, $S_{2 x}$, which is given by

$$
S_{\mathbf{H}}=\langle\mathbf{x}|\Delta U| \mathbf{k}\rangle
$$

is mathematically equivalent to the Fourier transformation of the perturbation potential feld in a real space into the 3-dimensional K-space. However, $\Delta U$ is essentially two dimensional tunction of $x$ and $y$, because the dilatation $\Delta(\mathbf{r})$. given by eq. 2-24, which constitutes $\Delta U$ is constant in the $z$-direction. Then the delta function comes out in the $K_{z}$-direction.

$$
S_{w}=\langle\mathbf{r}|\Delta U(x, y, x)| k\rangle
$$




$$
=2 \pi \delta\left(K_{z}\right)\left\langle\mathbf{k}^{\prime}|\Delta U(x, y)| \mathbf{k}\right\rangle_{2}
$$

where subscript 2 indicates the dimension and $K_{z}$ is the $z$-component of the vector $\mathrm{K}$ defined by

$$
\mathbf{K}=\mathbf{k}-\mathbf{k}
$$

Observing that the equi-energy surface for free electron is sphere surface, the Polar coordinate system is introduced (Fig. 5).

$$
\begin{aligned}
& k_{z}=k \cdot \sin \vartheta \cdot \cos \varphi \\
& k_{y}=k \cdot \sin \vartheta \cdot \sin \varphi \\
& k_{z}=k \cdot \cos \vartheta
\end{aligned}
$$

The surface integral appearing in the : 1.39 is, therefore, transformed to double integral with respect to $v^{\prime}$ and $\varphi^{\prime}$ for constant $k$-value. And the $\delta$-function singled out in the eq. $3-42$ innediately reduces it to single integral along the $\varphi^{\circ}$ lor constant $\vartheta$ value, ie. $v^{\prime}=\vartheta$, because

$$
\begin{aligned}
K_{1} & =k_{\mathrm{z}}^{*}-k_{\mathrm{a}} \\
& =k\left(\cos \gamma^{\prime}-\cos \vartheta\right)
\end{aligned}
$$

Now the Anal form of the B.T.E. can be given by

$$
\left(-\frac{\partial f^{0}}{\partial \varepsilon}\right) \theta \boldsymbol{r}_{\mathbf{k}} \cdot \mathbf{B}=\frac{\mathcal{L}(\mathbf{k})-f^{0}}{T}-C \int(f(\mathbf{k})-f(\mathbf{k}))\left|\langle\mathbf{K}|-8 \varphi| \mathbf{k}\rangle_{\mathbf{a}}\right|^{2} \mathrm{~d} \varphi^{\circ}
$$

where $C^{\prime}$ represents material and paysical constant.

As is shown in the original work of Mackenzie and Sondheimer [56], the solution of this integral equation can be found by successive approximation 
method (Neumann Series) [31] alter the following variable transtormatien.

$$
\begin{aligned}
G(\mathbf{k}, \boldsymbol{r}) & =f(\mathbf{k}, \boldsymbol{r})-f^{0}(\mathbf{r}) \\
& =-\frac{\pi}{m} \mathbf{g}(\mathbf{k}) \cdot \mathbf{4} \frac{\partial f^{0}}{\partial \varepsilon}
\end{aligned}
$$

and

$$
-\mathbf{k} \cdot \mathbf{B}=\frac{1}{\tau} \mathbf{R}(\mathbf{k}) \cdot \mathbf{k}+\left.C^{\prime} \int_{0^{\prime}}(\mathbf{c}(\mathbf{k}) \cdot \mathbf{k}-\mathbf{g}(\mathbf{k}) \cdot \mathbf{k})|<\mathbf{k}| \Delta U|\mathbf{k}\rangle_{2}\right|^{2} d \varphi^{\circ}
$$

where $C^{*}$ is again material and physical constant, and the integral equation is now posed for unknom function $g(\mathbf{k}) \cdot \mathbf{k}$ instead of the original distribution tunction $f(k)$. The result is given by

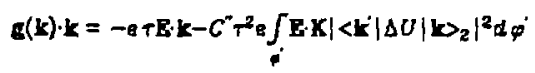

or

$$
\begin{aligned}
& \mathbf{g}(\mathbf{k}) \cdot \mathbf{k}=-\mathbf{e} \mathbf{T} \cdot \mathbf{k} \\
& -C^{-} \tau^{2} e \int_{p} \boldsymbol{B} K K_{\psi}^{2}\left(\frac{1}{\left(\gamma K_{z}\right)^{2}+K_{\gamma}^{2}}-\frac{2}{\gamma+1} \frac{1}{K_{z}^{2}+K_{y}^{2}+\alpha}\right)^{2} \alpha \dot{\phi}
\end{aligned}
$$

where the performance of Fourier transtormation on the two dimensional perturbation potential, $\Delta U$, leads the last equation 3-52. (APPENDIX B)

\section{III-2-2. Blectrical Resistivity}

By making use of the solution of B.T.F. obtained above, electrical resistivity is calculated. The calcuiation is started with the following definition of electron Dux, d. 


$$
J=-\frac{e}{4 \pi^{3}} \iint_{k} \int \boldsymbol{k} f(\mathbf{k}) d \mathbf{k}
$$

or

$$
J_{i}=-\frac{e}{4 \pi^{3}} \iint_{k} \int \nu_{i} f(k) d k \quad(i=x, y, z)
$$

Substitution of the eq. $3-49$ into the eq. $3-54$ followed by the applications of the eq. 3-40 and the next properties of equilibrium distribution function

$$
\iiint e v_{i} f^{a} d k=0
$$

and

$$
\frac{\partial f^{0}}{\partial \varepsilon}=-\delta\left(\varepsilon-\varepsilon_{p}\right)
$$

yields

$$
\begin{aligned}
& \mathcal{L}_{i}=-\frac{\dot{b}}{4 \pi^{3}}\left(\frac{\pi}{m}\right)^{2} \int\left\{\int k_{i} g(\mathbf{k}) \cdot \mathbf{k} \delta\left(\varepsilon-\varepsilon_{P}\right) d \mathbf{k}\right. \\
& =-\frac{g}{4 \pi^{3}}\left[\frac{\pi}{m}\right]^{2} \iint_{S_{i p}} \frac{k_{i}}{\hbar \nu_{i}} g(k) \cdot \operatorname{cod} S_{\mathrm{c}, p}(i=x, y, z)
\end{aligned}
$$

where the reduction of the integral is again based on the same procedures introduced previously in the eq. 3-39. It should be noticed that, in contrast to the universality of the condition 3-55, the eq. 3-56 is the approximate relation except for absolute zero Kelvin. This point is discussed in the next chapter $\mathbb{V}$ 3.

Electrical resistivity (conductinty) is essentially expressed as a tensor and each component, $J_{x}, J_{y}, J_{x}$, in the eq. 3-57 is responsible tor those tensor components. Mathematical extraction of the resistivity (conductivity) tensor components from the electron tux $J$ is demonstrated below for the case of $i=z$ ie. 


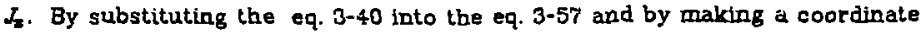
transformation from the Cartesian system to the Polar system, the next equation is obtained.

$$
J_{x}=-\frac{3 e n_{0}}{4 \pi m k} \int_{x} \int_{p} E(k) \cdot \sin \sin ^{2} v \cdot \cos \varphi \cdot d \vartheta d \psi
$$

where the geometrical relation among $v, \varphi$ and $k_{F}$ are given in the Fig. 5 , and $n_{0}$ is the electron density of perfect crystal which is related with Fermi vector, $k_{F}$. by

$$
n_{0}=\frac{k f}{3 \pi^{2}}
$$

Substitution of the eq. 3-52 into the eq. 3-58 yields two integrals.

$$
\begin{aligned}
J_{*} & =J_{z}^{(1)}+J_{z}^{(2)} \\
& =-A \rho T \int_{0}^{\pi} d v \int_{0}^{2 \pi} d \varphi E_{x} k_{x} \sin ^{2} \theta \cdot \cos \varphi \\
& -A C^{-1} \theta T^{2} \int_{0}^{\pi} d v \int_{0}^{2 \pi} d \varphi \int_{0}^{2 \pi} d \varphi E_{x} K_{z} F\left(K_{x}, K_{Y}\right) \sin ^{2} \psi \cos \varphi
\end{aligned}
$$

where $A$ and $F\left(K_{x}, K_{y}\right)$ are given by

$$
A=-\frac{3 m n_{0}}{4 \pi m k_{F}}
$$

and

$$
F\left(K_{x}, K_{y}\right)=K_{z}^{2}\left(\frac{1}{\left(\gamma K_{z}\right)^{2}+K_{y}^{2}}-\frac{2}{\gamma+1} \frac{1}{K_{z}^{2}+K_{j}^{2}+a}\right)^{2}
$$

Since the conductivity tensor (inverse of the resistivity), $\mathbf{Q}$, is deflned as 


$$
\mathbf{J}=\mathbf{n E}
$$

the tensor component $\sigma_{20}^{(1)}$ can be singled out from $J_{x}^{(1)}$.

$$
\begin{aligned}
\sigma(1) & =-A B \tau \int_{0}^{\pi} d v \int_{0}^{2 \pi} d \varphi k_{\rho} \sin ^{2} \vartheta_{\cos \varphi} \\
& =\frac{n_{0} e^{2} \tau}{m}
\end{aligned}
$$

In the same manner, although the calculation is complicated, $\sigma_{ \pm x}^{(2)}$ is obtaines from $J_{z}^{(2)}$.

$$
\begin{aligned}
\nabla(2) & =-A C^{\prime \prime} \mathrm{e} \tau^{2} \int_{0}^{\pi} d v \int_{0}^{2 \pi} d \varphi \int_{0}^{2 \pi} d \varphi K_{*} F(K) \sin ^{2} \theta \cdot \cos \varphi \\
& =-A C^{\prime \prime \prime} e \tau^{2} k_{F} C^{\infty} \pi^{2}\left(2+\left(\frac{\Gamma_{0}}{1-\Gamma_{0}}\right)^{2} \frac{p^{2}}{1+p^{2}}+p \frac{\Gamma_{0}\left(4-3 \Gamma_{0}\right)}{\left(1-\Gamma_{0}\right)^{2}} \sin ^{-1} \frac{1}{\sqrt{p^{2}+1}}\right)
\end{aligned}
$$

where $C^{\infty}, p$ and $\Gamma_{a}$ are given by

$$
\begin{aligned}
& C^{\infty}=-\frac{2\left(1-\Gamma_{0}\right)^{2}}{2^{2} k_{F}^{2}\left(\gamma^{2}\right)^{2}} \\
& p=\frac{\sqrt{a}}{2 k_{F}}
\end{aligned}
$$

and

$$
\Gamma_{0}=\frac{2 x^{2}}{1+\gamma}
$$

From the flrst line to the second one in the eq. 3-65, the following assumption ras employed.

$$
\gamma \sim 1
$$

of which validity is discussed later. For the other components, $y$ and $z$, the 
same manipulations lead to the following results.

$$
\begin{aligned}
& \sigma_{y}^{(1)}=\sigma_{x}^{(1)} \\
& \sigma_{w}^{(2)}=3 \sigma_{x z}^{(2)} \\
& \sigma_{x=}^{(1)}=\pi(1)
\end{aligned}
$$

and

$$
\sigma \stackrel{(2)}{s}=0
$$

The final step is an elegant applications of the Matthiesen's rule [32]. According to the Matthiesen's rule the resistivity is given as the surn of two contributions. One is the contribution of lattice vibration which depends on temperature and the other one is caused by impurities, defects and so on which is generally independent of temperature. Thus, mathematically, this rule is described by

$$
\frac{1}{\sigma_{x}}=\frac{1}{\sigma_{i}}+\frac{1}{\sigma_{x}}
$$

where the first term of the right hand side is the resistivity of lattice and the second one is that of the dislocation which is the final object of this calculation. $\sigma_{i}$ is easily derived from fundamental solid state electronics [33] and is given by

$$
\sigma_{i}=\frac{n_{0 \theta^{2}} T}{m}
$$

One would notice that this is nothing more than $\sigma_{z \neq}^{(1)}, \sigma_{y y}^{(1)}$ and $\sigma_{* x}^{(1)}$ obtained above. Here the following assumption is introduced: the lattice contribution is much greater than that of the dislocation.

$$
\sigma_{i} \ll \sigma_{3} \text { ie. } \frac{1}{\sigma_{i}} \gg \frac{1}{\sigma_{z}}
$$


Then, from eq. 3-74, $\sigma_{x=}$ is approximated to be

$$
\sigma_{c x} \sim \sigma_{i}-\frac{\sigma_{i}^{2}}{\sigma_{z}}
$$

and by making use of the above eq.3-77 with

$$
\sigma_{z x}=\sigma_{2}^{(1)}+\sigma_{z=}^{(2)}
$$

and

$$
\sigma_{m}^{(1)}=\sigma_{v}
$$

the dislocation resistivity, $\rho_{z}$, can be extracted.

$$
\begin{aligned}
\rho_{x} & =\frac{1}{\sigma_{x}} \\
& =-\frac{\sigma_{x}^{(2)}}{\left(\sigma_{I I}^{(1)}\right)^{2}}
\end{aligned}
$$

Like wise

$$
p_{y}=3 p_{x}
$$

and

$$
\rho_{x}=0
$$

Although substitution of eqs.3-64 and 3-65 makes the above equation more realist1e, this task is accomplished in the next chapter.

\section{III-3. Priction Porce.}

In the previous section it was demonstrated that a moving edge disiocation line causes electrical resistivity as an intrinsic property. On the other hand, 
during the motion with velocity $v$, the dislocation experiences the electris current. $i$, caused by positive ion charges. As a result Joule beat energy is expected to be dissipated. This energy should be supplied externally in order to keep the steady motion. The friction lerce on a moving edge dislocation originates from this energy loss and is estimated in the following manner.

The confguration in the Fig. 6 is considered. According to the well known Joule-henz Law, the heat energy dissipated by the current $i$, in an object of resistivity $R$, during time $t$, is given bj

$$
\begin{aligned}
\text { Qhoet } & =i^{2} R t \\
& =i^{2} \rho \frac{L_{0}}{A_{0}} t
\end{aligned}
$$

The electric current, $i$, experienced by the dislocation of velocity, $v$, is

$$
i=\min _{0} A_{0} v
$$

where $A_{0}$ is the transverse area of the model sample shown in the Fig. $B_{\text {, and }}$ is given by

$$
A_{0}=m
$$

and time. $t$, is also easily calculated.

$$
t=\frac{L_{0}}{v}
$$

Substitution of the eqs. 3-84 3-8B into the eq. 3-83 yields

$$
\text { Qheat }=a^{2} n_{0}^{2} v \rho L_{0}^{2} A_{0}
$$

Let $\boldsymbol{F}^{*}$ be the applied forcs on a dislocation of unit length to keep the steady motion, which is equivalent to the triction lorce. Then the following relation is 
obtained.

$$
F^{*} L_{0}=\text { Qhow }
$$

And from eqs. 3-87 and 3-88, the friction force per unit length is estimated to be

$$
F^{*}=e^{2} n_{0}^{2} v_{\rho}
$$

By substituting the previously obtained results of electrical resistivity and the appropriate physical c-astants into the eq. 3-89, the friction force is explicitly obtained as

$$
F^{*}=5.07 \times 10^{*}\left\{b^{2}\left(\frac{v_{l}}{v_{l}}\right)^{4} v_{l}\right\} G^{*}(p, y)
$$

and

$$
G^{*}(p, \gamma)=\frac{\sqrt{1-\gamma^{2}}}{(\gamma)^{2}} \frac{1}{p^{20}}\left(2\left(1-\Gamma_{0}\right)^{2}+\Gamma_{0} \frac{p^{2}}{p^{2}+1}+p \Gamma_{0}\left(4-3 \Gamma_{0}\right) \sin ^{-1} \frac{1}{\sqrt{p^{2}+1}}\right)
$$

where $p$ and $\Gamma_{0}$ were given in the eqs. 3-67 and 3-68.

\section{III-4. Yagnetic Induction}

Magnetic induction is the dynamic effect of moving charges. In a strict manner, it should be derived as the solution of the Maxwell's equations 2-28 231. In this study, bowerer, all the treatments so far have been limited to the quasi-statical treatment in which the vector potential is ignored and the problem is posed as the effect of "moving electrostatic notential". Then, within the trame work of this model. the following resits are not expected to satisty Maxwell's equations rigorously and the calculation itself may not be compatible with the model, In spite of this basic refutation, however, it is still considered 
to be of consequence to estimate the magnetic induction because ot the following two reasons. First of all, the results will provide the limitation of the quasistatic treatment: if the estimated magnetic induction is not negligibly small, the spatial density of electrons which was given by the eq. 3-19 should be modifled by returning to Jaxwell's equations so that the vector potential is incorporated in the model. Secondly, the possibility of a new type of interaction between the magnetic Aeld around a moving dislocation and the magnetic moments of magnetic ion clusters in the crystal can be examined.

The procedure adopted here is rather simple and straightforward. The basic origin of the magnetic induction is the uotion of the electron cloud associated with the moving dislocation line. Instead of applying the full expression of the electron density eq. 3-19, the limiting case of infinite screening constant. $\sqrt{a}=\infty$, is calculated.

The magnetic induction caused by a moving charge. Qenerg, is generally given by

$$
\begin{aligned}
B & =|B| \\
& =\frac{\mu_{0}}{4 \pi} \frac{Q_{c \text { carrge }} y}{r^{2}\left(1-\frac{y^{2}}{c^{2}}\right)^{2}}
\end{aligned}
$$

where $\mu_{a}$ is the permeability constant and $r$ is the distance between the charge Qcker and an observing point. As mentioned belore, the speed of a dislocation. $v$, is very much smaller than that of light, $c$. Therefore, the above equation can be approximated as

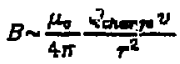

A more general yect,or description of the magnetic induction for the moving charge in the $z$-direction is given by 


$$
\begin{aligned}
B & =B_{i}\left|e_{i}\right\rangle \quad(i=x, y, z) \\
& =-\frac{\mu_{0} v Q_{\text {charge }}}{4 \pi r^{3}} r_{z}\left|e_{y}\right\rangle+\frac{\mu_{0} Q_{\text {ehargz }} y}{4 \pi r^{3}} r_{y}\left|e_{z}\right\rangle
\end{aligned}
$$

where $B_{i}$ and $\left|e_{i}\right\rangle$ are the component of the uiagnetic induction and the unit vector in the $i$-direction, respectively. For simplicity the present calculation is pertormed only on the $x-y$ plane on which the $B_{y}$ component vanishes because of the symmetry of the charge distribution, and only the $B_{2}$ component

$$
B_{x}=\frac{\mu_{0} Q_{c h a r g e} v}{4 \pi r^{3}} r_{y}
$$

remains.

By applying this elementary principle to the moving electron cloud associated with the edge dislocation. the following calculation is carried out. The induction. $\Delta \theta_{z}$, at the positisn $P(x, y, 0, t)$ caused by the electron clouds residing io tine voilizne element $\left(x_{0}^{\prime}+\Delta x_{i}^{\prime}, y_{j}^{\prime}+\Delta y_{j}^{\prime}, z_{k}^{\prime}+\Delta z_{k}^{\prime}\right)$ is given by

$$
\begin{aligned}
& \Delta B_{2}\left(x_{i}, y_{j}, x_{k}^{\prime}, t\right) \\
& =\frac{\mu_{0} v}{4 \pi} \frac{y-y_{j}^{j}}{\left\{\left(x-x_{j}\right)^{2}+\left(y-y_{j}\right)^{2}+\left(x_{k}\right)^{2}\right\}^{3 / 2}} \frac{(-\theta) C_{1} \gamma y_{j}^{j}}{\left\{\left(x_{i}-v t\right)^{2}+\left(\gamma y_{j}\right)^{2}\right\}} \Delta x_{i} \Delta y_{j} \Delta x_{k}
\end{aligned}
$$

where the second traction term expresses the space term. $\frac{T_{y}}{\tau^{3}}$, in the eq. 3-94. the third traction term is the electron density which is calculated in eq. 3-19 and $C_{1}$ is the physical constant given by

$$
c_{1}=-\frac{b}{15} \frac{1}{\pi^{3}}\left(\frac{2 m}{n^{2}}\right)^{5 / 2}\left(\frac{1-2 \nu_{0}}{1-\nu_{p}}\right) \varepsilon_{F}^{03 / 2}
$$

Then the final formula for the magnetic induction $B(x, y, 0, t)$ is obtained by integrating the eq. 3-96 over the crystal in the following way. 


$$
\begin{aligned}
& B_{z}(x, y, 0, t)=\lim _{\Delta x_{i} \Delta y_{j}, \Delta x_{k}^{\prime} \rightarrow 0} \sum_{i} \sum_{j} \sum_{k} \Delta B_{z}\left(x_{i}, y_{j}^{\prime}, z_{k}^{\prime}, t\right) \Delta x_{i} \Delta y y_{j} \Delta x_{k}^{\prime} \\
& =-\frac{e \mu_{0} v}{4 \pi} C_{1} y \iiint \frac{y-y^{\prime}}{\left\{\left(x-z^{\prime}\right)^{2}+\left(y-y^{\prime}\right)^{2}+\left(x-z^{\prime}\right)^{2}\right\}^{3 / 2}} \frac{y^{\prime}}{\left(x^{\prime}-v t\right)^{2}+\left(\gamma y^{\prime}\right)^{2}} d z^{\prime} \cdot d y^{\prime} \cdot d z^{\prime}
\end{aligned}
$$

The actual integration of the above eq. 3-98 was pertormed numerically after the reduction of the triple integral to the single integral. 


\section{RPSULIS AND DISCUSSION}

\section{IV-1. Blectron Density and Perturbetion Potential}

In the former chapter, the electrostatic potential was obtained in eq. 3-16 with the Green's lunction $g_{2}(\mathbf{r} \mid \xi)$. and the third term could be neglected by the numerical evaluation. This numerical process is briefly examined beiore the discussion of the physical signiflcance of the calculated electron density and electrostatic potential.

By excluding the common constant term from each term, the electrostatic potential, $\varphi(\mathbf{r})$, is given by the sum of three terms.

$$
\begin{aligned}
\varphi(\mathbf{r}) & =-\frac{D}{a}\left(\Delta(\mathbf{r})-2 C \sqrt{a} K_{1}(\sqrt{a}|\mathbf{r}|) \frac{\sin v}{\gamma+1}+\iint_{b_{3}} g_{2}(\mathbf{r} \mid \xi) \nabla^{2} \Delta d \xi\right) \\
& =-\frac{D}{a} C\left(I_{1}+I_{2}+I_{g}\right)
\end{aligned}
$$

where

$$
\begin{aligned}
& I_{1}(x, y)=\frac{n y}{x^{2}+(\gamma y)^{2}} \\
& I_{2}(x, y)=\frac{2 \sqrt{a}}{\gamma+1} K_{1}\left(\sqrt{a} \sqrt{x^{2}+y^{2}}\right) \frac{y}{\sqrt{x^{2}+y^{2}}} \\
& I_{s}(x, y)=\frac{\gamma\left(1-y^{2}\right)}{\pi} \iint_{K_{u}} K_{0}(\sqrt{a}|\mathbf{r}-\xi|) \frac{\xi_{y}\left(3 \xi_{z}^{2}-\gamma^{2} \xi_{y}^{2}\right)}{\left(\xi_{z}^{2}+\gamma \xi_{y}^{2}\right)^{3}} d \xi, d \xi_{y}
\end{aligned}
$$

Each contribution was plotted in Figs. 2. 3, and 4 for various peints $\left(x^{\prime}, y^{\prime}\right)$. The employed velocities are $0.25 v_{l}(\gamma=0.968)$ and $0.5 v_{l}(\gamma=0.665)$ which is the possible maximum velocity, and the screening constant, $\sqrt{a}$, is fixed at $1.5 \times 10^{\circ}$. (The validity of this adopted value of screening constant is demonstrated in the discussion. eq. 4-15.) It can be clearly seen that the essential contribution is 
attributed to the first term both for tine diagonal direction and on the circle, and the magnitude of the third term is signiflcantly smaller than those of ather terms. In the light of these numerical results the third term was neglected in the calculation prucedures. isthough the physical meaning at this term is still open to question, it should be noticed that the dilatation, $\Delta$, becomes a harmonic function for a static dislocation and ther srore the third term spontaneously vanishes. This tact implies that the thitd term represents a dynamic effect. Probably strict solution of the Maxrell's equations will settle this ambiguity satistactorily.

Now attention is turned to the calculated electron density. The eq. 3-19 is split into two terms and the physical meaning of each term is considered.

$$
\begin{aligned}
\rho(\mathbf{r}) & =\rho_{0}\left[1-\Delta+\frac{4}{5} C \sqrt{a} X_{1}(\sqrt{a}|\mathbf{r}|) \frac{\sin \vartheta}{\gamma+1}\right) \\
& =\rho_{1}(\mathbf{r})+\rho_{2}(\mathbf{r})
\end{aligned}
$$

where

$$
\begin{aligned}
\rho_{1}(r) & =\rho_{0}(1-\Delta) \Delta \ll 1 \\
& =\rho_{0}(1+\Delta)^{-2}
\end{aligned}
$$

and

$$
\rho_{2}(r)=\rho_{0}\left[\frac{4}{5} C \sqrt{a} K_{1}(\sqrt{a}|r|) \frac{\sin s}{\gamma+1}\right]
$$

Since $(I+\Delta)^{-1}$ expresses the lattice dilatation the frst term, $p_{1}(r)$. indicates the electron density whose shilt is accompanied with positive ions on the lattice points. In this sense these electrons are tightiy bound to the positive lons like 
an ionic crystal. The essential leature of a metal is refected in the second term. $\rho_{2}(\boldsymbol{r})$, which is the contribution of the screening effect of free electrons. In order to show these irro effects schematically, the following modifcations are performed on the eq. $3-13$. The dilatation, $\Delta$, was given by

$$
\Delta=C \frac{y y}{x^{2}+(y y)^{2}}
$$

where

$$
C=\left(-\frac{b}{2} \pi\right) 2\left(\frac{v_{s}}{v_{l}}\right)^{2}
$$

Then the eq. 3-19 is rewritten as

$$
\frac{\rho(\mathbf{r})-\rho_{0}}{\rho_{0} C^{0}}=\frac{\gamma y}{x^{2}+(\gamma y)^{2}}-\frac{4}{5} \frac{1}{\gamma+1} \sqrt{a} \sqrt{x^{2}+y^{2}} K_{1}\left(\sqrt{a} \sqrt{x^{2}+y^{2}}\right) \frac{y}{x^{2}+y^{2}}
$$

where

$$
c^{*}=-C
$$

Tae eq. 4-10 describes the change of the density rather than density itself. The screening term $\sqrt{a}$ was more explicitly gigen by eq. $3-12$.

$$
\begin{aligned}
a & =\frac{6 \pi e^{c} \rho_{0}}{\varepsilon_{\min }^{0}} \\
& =1.969 \times \mathrm{e}^{2} \frac{2 m}{n^{2}} \rho_{0}^{j}
\end{aligned}
$$

and has the dimension $\left[L^{-1}\right]$. Substitution of appropriate physical constants into the above equation yields,

$$
a=7.498 \times 10^{8} p_{0}^{\frac{1}{3}} \text { [c.g.s.] }
$$


Since the electron density, $\rho_{0}\left(=n_{0}\right)$, ol a typical metal resides in the range of

$$
10^{22} \leq \rho_{0} \leq 2 \times 10^{23}
$$

the screening constant is conflned to the following range.

$$
1.616 \times 10^{16}\left[\mathrm{~cm}^{-1}\right] \leq a \leq 4.386 \times 10^{10}\left[\mathrm{~cm}^{-1}\right]
$$

The performance of the following scaling

$$
\begin{aligned}
& x[\mathrm{~mm}]=10^{-8} X \text { [angstrom] } \\
& y[\mathrm{~cm}]=10^{-8} Y \text { [angstrom] } \\
& \left.\sqrt{a}\left[\mathrm{~cm}^{-1}\right]=10^{8} \sqrt{a} \text { [angstrom }{ }^{-1}\right]
\end{aligned}
$$

tronsforms eq. 4-10 into the fnal desired form

$$
\frac{\rho(\mathbf{r})-\rho_{0}}{\rho_{0} C^{0} 10^{8}}=\frac{\gamma Y}{X^{2}+\left(\gamma^{Y}\right)^{2}}-\frac{4}{5} \frac{1}{\gamma+1} \sqrt{a} \sqrt{X^{2}+Y^{2}} K_{1}\left(\sqrt{a^{3}} \sqrt{X^{2}+Y^{2}}\right) \frac{Y}{X^{2}+Y^{2}}
$$

The contributions of the first term, the second tern and the whols term are schematically plotted in Figs. $?, 8,9$ and 10 for various values of $y$ and $\sqrt{a}$. (Since the first term is independent of the screening constant, $\sqrt{a}$, the contribution of this term is shown as the function of three values of velocities, $v / v_{t}=0.0 .0 .25$ and 0.5 in the Fig. 7. The dependencies of the second term and whole term on three types of screening constant. $\sqrt{a}=1.0,1.5,2.0$, are plotted in the Figs. 8, 9, and 10, respectively, for the three kinds of velocities. "GM" and "SCR" in each Agures stand for $\gamma$ and $\sqrt{a}$. Scales are units of Burgers vector.) The dark part indicates a higher electron density than the light part. It is immediately understood that the screening contribution is a localized efect and that total electron density is determined primarily by the first term, which is nothing more than the equivalent consequence of the lormer calculations of 
electrostatic potential given in the eqs. $4-2 \sim 4-4$. One should notice that the electron density is antisymetric with respect to $x$-axis and blows up at the origin. This explosion at the origin is observed in the electrostatle potential as well and therelore in the perturbation potential as is shown in the Figs. 11,12 and 13. The origin of this physicaliz inallowable phenomenon is the direct consequence of the application of linear elasticity argument to the core portion. which was introduced in the section II-2. It is well recognized that the atomic arrangement of the core can not be predicted by the linear elasticity theory. There might be some relaxation process in the core and there must be more suitable smooth function which deseribes the real strain or dilatation in the eore portion. Since progress has been gradually made in the theld of "core physics" mainly based on computer simulation study [34] and quantum mechanical approaches[35], the improvement and modification of the core portion by applying non-elasticity arguments remains as indispensable task in the Puture.

\section{IV-2. Born Approrimation}

The Born approximation (APPENDIX A) adopted in this calculation is one of the typical classical treatments of perturbation theory. In this treatment, the wave lunction of the corresponding perturbed Hamiltonian is approximated to that of the perfect system, and only "small" perturbation potentials can be well described by this theory. In the present study. magnitude of the perturbation potential given by eq. $3-35$ is about $8.4 \times 10^{-3} \varepsilon_{k i n}^{\mathrm{d}}$ at $(5 b, 5 b)$ lor the case of $k_{F}=1.5 \times 10^{8}, b=3.0 \AA$ and $\gamma=0.1$. This value may be lairly small enough to satisfy the criterion of the Born Approximation. However, as shown in the Fig. 11. perturbation potential blows up approaching toward the origin. Therefore the direct application of the Bom Approximation to a whole crystal must be open to 
objections. There could be a more suitable treatment for this type of strong scattering potential celd. But the essential point is not the impropement or modification of the approximation method but to obtain the proper scattering potential Geld based on the proper atomic arrangement in the core portion.

IV-3. Boltzman Transport Equation and Mackenzie-Sondheimer's procedures.

The key procedure of the Mackenzie-Sondheimer's B.T.E. method is the dextrous application of the Matthiesen's rule in the anal stage. It is not too much to say that the mathematical elegance of the whole method is amplified by this procedure. This powerful method, however, includes the following selfcontradiction. The Anal process to obtain the resistivity value was based on the assumption:

$$
\sigma_{i} \ll \sigma_{x} \text { ie. } \frac{1}{\sigma_{i}} \gg \frac{1}{\sigma_{z}}
$$

which means the resistivity of the lattice is very much bugher than that of the dislocation. Since the relaxation time $\tau$ of lattice resistivity decreases inversely proportional to the temperature and according to Matthlesen's rule the resistivity of dislocations is independent of temperature, the assumption eq. 3-76 should describe the bigher t:=mperature state. On the other hand, once returned to the reduction process Irom eq. 3-54 to eq. 3-57, one realizes that the mathematical property of the Fermi-Dirac distribution function, eq. 3-56, was an essential ingredient. But this tunctional property is, in a rigorous sense, limited to $0^{\circ} \mathrm{K}$. This self-contradiction seems to violate the physical validity of this method.

In order to extract the pure contribution of a dislocation at $0^{\circ} K$, the numerical calculation was carried out on the following integral equation 4-18 which is obtained from eq. $3-48$ in the limit of inflnite relaxation time (The 
mathernatical detail is shown in the APPENDIX C.)

$$
-a \mathbf{k} \cdot \mathbf{E}=C^{\prime \prime} \int_{\dot{f}^{\prime}}\left(\mathbf{g}(\mathbf{k}) \cdot \mathbf{k}-\mathbf{g}(\mathbf{k}) \cdot \mathbf{k}^{\prime}\right)\left|\left\langle\mathbf{k}^{\prime}|-a \varphi| \mathbf{k}\right\rangle_{2}\right|^{2} d \varphi^{\prime}
$$

Comparison ol the numerical results with analytical results is made in the Table 1 for the case of a static dislocation, $y=1$, and $\sqrt{a}^{x}=1.5$. (In Table 1, coeflecient term is omitted and only $k_{F}$-dependent values are listed.) It can be understood that, although the analytical values are slightly higher than the numerical values which give the real dislocation resistivity, the Mackenzie-Sondheimer's analytical procedures provides a tairly good estimation of dislocation resistivity. The origin of this good agreement can be attributed to the following lunctional property of the Fermi-Dirac distribution tunction: The Fermi-Dirac distribution function is an exact step tunction at $0^{\circ} \mathrm{K}$, and deviation from the step lunction is negligibly small even at the higher temperatures. This guarantees the validity of the eq. 3-56 in the wide temperature range.

\section{IV-4. Friction Foree and Mlectrical Resistivity}

The theoretical friction force was given by the eqs. 3-90 and 3-91.

$$
\begin{aligned}
& F^{*}=5.07 \times 10^{\circ}\left(b^{2}\left(\frac{v_{3}}{v_{l}}\right)^{4} v_{i}\right) G^{*}(p, \gamma) \\
& G^{*}(p, \gamma)=\frac{\sqrt{1-\gamma^{2}}}{\left(\gamma^{2}\right)^{2}} \frac{1}{p^{10}}\left(2\left(1-\Gamma_{0}\right)^{2}+\Gamma_{0} \frac{p^{2}}{p^{2}+1}+p \Gamma_{0}\left(4-3 \Gamma_{0}\right) \sin ^{-1} \frac{1}{\sqrt{p^{2}+1}}\right)
\end{aligned}
$$

These results are plotted in the Fig. 14 as a function of velocity lor various values of Fermi vector, $k_{F}$ [38]. It is understood that damping constants which can be calculated from the slope of the curve is, in general, a function of velocity. However, as shown in the Flg. 15 . force is linearly related with velocities for their physically meaningful values. And damping constant is independent of 
velocity. In order to estimate the actual values predicted by this theoretical calculation, physical and materials constants which are tabulated in the Table 2 are substituted into the above equations, and damping constants are estimated for $\mathrm{Cu}, \mathrm{Pb}$, and $\mathrm{Al}$. The results are tabulated in the Table 3 . It is needless to say that the accuracy of these values and the validity of the theory can be examined only by comparison with the experimental values. However, it is not an easy task to experimentally extract the friction force and damping constant with accuracy suffient enough to allow comparison with theoretical values. A sample should not bave high Peierls potential fleld so that the tree night dislocation molion is a dominant mode, and the temperature should be low enough to aroid the phonon friction force. In the case of a tensile test, in addition to these requirements for the sample and experimental condition, a more essential diffeulty arises in the following manner.

A tensile tesi is one of the most widely used experimental procedures for the mechanical testing of materials. In fact, as was mentioned previousiy, the exdstence of the electron friction force was first predicted by this type of experiment. But in order to obtain the friction force from the observed stress change due to the superconducting and normal transition, the following theoretical procedures are required. The strain rate of a sample. $\dot{\varepsilon}$, is generally given by

$$
\bar{\varepsilon}=N_{m} b \bar{y}
$$

where $N_{m}$ is the mobile dislocation density, $\bar{v}$ is the average velocity of those dislocations and $b$ is the magnitude of the Burgers vector. When the rate controlling process is assumed to be a thermally activated process the velocity can be rewritten as

$$
v=\frac{L_{1}}{t_{r}}
$$




$$
=L, \nu \exp \left(-\frac{\Delta G^{*}}{k T}\right)
$$

where $\Delta G^{*}$ is the activation tree energy: Lis the average distance among obsta* cles; $v$ is the trial frequency and $t_{y}$ is the waiting time for the thermal activation at an obstacle. The activation tree energy can be more explicitly given by

$$
\Delta G^{*}=G_{a}-\left(\tau_{\text {qpp }}-\tau_{L}\right) V_{a c t}
$$

where $G_{\mathrm{o}}$ is the interaction energy between a dislocation and an obstacle; $\tau_{\text {app }}$ and $\tau_{L}$ are the applied lorce and long range internal back stress due to other dislocations, respectively, and $V_{\text {act, }}$ is the activation volume. Substitution of the eqs. $4-21$ and $4-22$ into the eq. $4-19$ yields

$$
\dot{\varepsilon}=N_{m} b L v \exp \left(-\frac{G_{g}-\left(\tau_{a p p}-\tau_{l}\right) V_{a x t}}{k T}\right)
$$

From the above equation, the applied stress, $\tau_{\operatorname{cop}}$, can be obtained as

$$
\tau_{a p P}=\tau_{L}+\frac{G_{0}}{V_{a x t}}-\frac{k T}{V_{a x t}} \ln \left(\frac{\dot{\varepsilon}}{N_{m} b L_{L} V}\right)
$$

By performing this procedure on both the normal and superconducting states, and by assuming that the long range internal stress, activation volume and mobile dislocation density do not change with the transition, the observed stress change due to the transition can be deseribed by the following quantities.

$$
\begin{aligned}
\Delta \tau & =\left(\tau_{\text {qqp }}\right)^{n}-\left(\tau_{\text {app }}\right)^{3} \\
& =\left(\tau_{e}\right)^{n}-\left(\tau_{0}\right)^{s}
\end{aligned}
$$

where superscript $n$ and $s$ indicate the normal and superconducting states, respectively. Since the electron triction force $\tau_{0}$ can be related with the 
damping constant $B_{a}$ and the velocity $v$ by

$$
\tau_{a}=\frac{B_{a} v}{b}
$$

the eq. 4-26 can be rewritten as

$$
\begin{aligned}
\Delta T & =\frac{v}{b}\left(B_{\theta}^{n}-B_{\theta}\right) \\
& =\frac{v}{b} B^{n}(1-\Gamma)
\end{aligned}
$$

where $\Gamma$ is

$$
\begin{aligned}
\Gamma & =\frac{B_{c}^{4}}{E^{n}} \\
& =\left(\frac{T}{T_{C}}\right)^{4} \text { (twa fluid smadal) }
\end{aligned}
$$

or

$$
=\frac{2}{1+\exp \left(\Delta_{g} / k T\right)} \text { (B.C.S. theory) }
$$

Then, as the product of damping constant $B$ and velocity $v$, the electron triction force in the normal state, $F^{n}{ }^{n}$, can be obtained from the eq. 4-29,

$$
\begin{aligned}
F_{b}^{2} & =B_{p}^{2} v \\
& =\frac{b}{1-\Gamma} \Delta t
\end{aligned}
$$

or from eqs. $4-28$ and $4-30 . \mathrm{Fe}^{3}$ is

$$
F=B
$$




$$
=\frac{\Gamma}{1-\Gamma} b \Delta t
$$

In the above calculations, the interaction force is obtained as only a function of temperature and measured stress change. However, this procedure is much too roundabout a process and many unclarifed assumptions are piled up. In fact, there is no physical basis that can guarantee the assumption of invariability of the $\tau_{L}, G_{0}$ or $V_{\text {act }}$ between the two states. Estimation of the arerage distance $L_{2}$ appeared in the eq. 4-23 telongs to the statistical problem of hardening which was introduced in chapter I-1. But the extension of the statistical problem of a static dislocation to that of a dynamic dislocation has not yet been satisfactorily investigated. Also, as was mentioned in chapter $5-3$, it is reported that, in ihe superconducting state, a dislocation which moves with a velocity higher than some critical velocity destroys the condensed electron pair and an additional friction force is exerted.[12] This microscopic physical property of the superconducting state is not taken into account in the above treatment. The effect of the magnetic field on the sample, which must be applied in order to pull the sample back to the normal state below critical temperature, is still open to question. Under the magnetic teld, careless experimental alignment often does not make the testing machine behave as a mechanical reservoir. At any rate, even apart from these shortcomings, the mos: serious problem in the above treatment resides in the lact that raw data does not reflect the desired quantity directly and that the intervention of unclarifled theories into the data interpretation process can not be avoided. Therefore one should conclude that it is next to impossible to extract a reliable value of a microscopic quantity such as the electron triction force Irom macroscopic values determined from a tensile test. In order to obtain reliable values of the friction force other experimental techniques must be gourint. 
Essentially three different approaches have been taken towards measuring the damping constant. One is the direct measurement of dislocation velocity as a function of applied stress by means of etch-pit technique; another one is the impact shear stress test which requires an assumption of a value for the mobile dislocation density; the other one is the measurement of ultrasonic attenuation. At this stage, it is recognized that the measurement of ultrasonic attenuation is the more reliable technique. The method proposed by Hikata et. al. [37] does not depend on a knowledge of the dislocation density and other inaccuracy resulting trom dislocation networks. The details of their lechnique are not given here but their reported values of electron damping constant are tabulated in Table 3 with theoretical values including those obtained by present study. (Victoria et.al.'s measurement is based on impoct shear tests. ) Agreements between the values calculated in this work and the measured valueg are not excellent, but as a whole fairly good agreements are achieved.

Although it was mentioned that ultrasonic attenuation measurement was the most reliable available method, the obtained data must still be manipulated by theoretical calculationg based on unfirmed assumptions. In this respect, the ultrasonic attenuation method is also an indirect measurement. By the way. as was demonstrated in the previous chapter. the essential physical basis behind this calculation was attributed to the Joule heat energy dissipation due to the electrical resistivity of a dislocation. Then, by digressing from the fnal calculation of friction force tor a while, it is considered to be of great haportence to tocus on a comparison of the theoretical electrical resistivity value of a static dislocation with experimental values which can be much more directly measured than the friction force and, therefore, more reliable.

In order to select pertinent msasured values of the resistivity for comparison, Matthiesen's rule is re-examined. This rule says that the electrical 
resistivity, $\rho_{\alpha}(N, T)$, due to $V$ dislocations at temperature $T$ is given by

$$
\rho_{d}(N . T)=\rho_{p}(T)+\rho_{d}(N)
$$

where $\rho_{p}(T)$ is the resistivity of pure crystal due to the lattice vibration and $\rho_{4}(N)$ is the contribution of $N$ dislocations which does not depend on temperature. With the progress of measurement techniques, however, the existence of extra term. $\triangle p_{D U R}(N, T)$, such as

$$
\rho_{d}(N, T)=\rho_{p}(T)+\rho_{d}(N)+\Delta \rho_{D U R}(N, T)
$$

has been reported $[38,39]$ and $\triangle_{0, R}$ is named DMR (Deviation from Mattbiesen's Rule). Since the construction of the present study is fully based on the Matthiesen's rule the experimental value which is compared with present theoretical results should not be affected by this uninvited DMR term. The main factors contributing to the DMR term are considered to be the development of cellular structures and the change of the phonon spectrum (vibration mode) due to the introduction of dislocations. Therefore, in order to eliminate DMR contributions, the experimental value should be selected trom a sample rhich bas a lom dislocation density so that the lattice vibration mode is not very much perturbed, and has uniform distribution of dislocations mithout having substructures. Moreover, a low temperature measurement is required to suppress the lattice contribution. $\rho_{p}(T)$. To the author's knowledge, there is only one available experimental result which satisfles the above condition. This experiment ras performed by Rider et. al. $[40\}$ on a poly $\mathrm{Al}$ crystal at liquid He temperature. In the Al gample, through the whole range of their measurement up to $15 \%$ strain. they observed a proportional relationship between resistivity and dislocation density (determined by TEM), and concluded that the additional contribution arising from confguration or density was avoided. Their measured 
resistivity

$$
p_{\sigma}=(1.8 \pm 0.1) \times 10^{-10}\left[\mathrm{ohm}-\mathrm{cm}^{3}\right]
$$

is adopted to compare with the present calculations whose general dependencies on velocities and Fermi vectors are shown in Fig. 16. Substitution of the material constants into the eqs. $3-64,3-65,3-80$ and $3-81$ followed by the modifleation for the poly crystal (APPENDIX D) yields

$$
p_{\text {th }}=1.02 \times 10^{-21}\left[\mathrm{ahm}-\mathrm{cm}^{3}\right]
$$

which is about two order lower than experimental valus. Pex, eq. 4-37. This theoretical value is slightly modifled by the following corrections. According to the measurements and calculations of electronic speciff heat. the effectire mass of a conduction electron, $m^{*}$, in Al crystal is estimated to be

$$
m^{*}=1.48 m_{0}
$$

where $m_{0}$ is the static mass. Substitution of this relation into the eqs. 3-84 365 immediately makes small corrections on the calculated value, iq. 4-3B.

$$
p^{*}=1.06 p
$$

This is, probably, the theoretically highest obtainable estimation in this model. Although even this correction is not sufleient to th the gap between the calculated and weasured values this amount of discrepancy is not unexpected because of the following reason. Since the sample used was poly crystal there must be additional contributions of grain boundaries. The vacancies which are introduced during the deformation should add the extra contributions, as well. Then the experiment provides overestimated value as compared with the true contribution of dislocation Itself. 
Therelore one can conclude that this simple model gives a fairly good estimation of the resistivity. Moreover, as will be shown in the next section, the effect of the magnetic field -- the dynamic effect -- is negligibly small, then the model of triction force, which is simply extended as the quasi-static motion of sharging sloud. can also provide the reliable estimation. And the result of eqs. 3-90 and 3-91 may be understood to be fairly reliable.

\section{IV-5. Magnetic Indaction and Yagnetic Interaction}

Fy substitutiog appropriate material and physical constants tonto the eqs. 3-97 anc 3-98, the magnetic induction $B_{3}(x, y, 0,1)$ is calculated as a function of dislocation velocity. In the Fig. 17. the spatial distribution of the integral part of the eq. 3-98 which depends only on the coordinates $(x, y, z),\left(x^{\prime}, y^{*}, z^{\prime}\right)$ and $\gamma(v)$ is showa for the case of $v / v_{t}=0.3$. In the Fig. 18, the same quantities are plotted as the tunction of $y$ coordinates for the constant $x$ value, $x=5 b$, and three kinds of velocities. One can see that the magnetic induation blows up approaching to the origin: this has the same physical origin as the singularity observed for elestron density or perturbation potential feld. Substitution of the actual material constant into the above equation yields the magnitude of $10^{-35} \sim 10^{-36}$ (Tesla/102b) tor both Ag and Au on the radius of $5 b$. These values are small enough to conclude that the effect of magnetic induction is virtually negligible.

As a very rough approximation, the magnitude of the magnetic interaction energy can be estimated, as well. The interaction energy between the magnetic induction, $B$, and the magnetic moment, $\mu$, is given by [ $A 1]$

$$
E_{\operatorname{mag}}=\mathbf{B} \boldsymbol{\mu}
$$

In the true sense, however, the magnetic induction $\mathbf{B}$ produced by the moving 
dislocation is not independent but is varying with the dislocation motion. Then the interaction process with the "static" magnetic moment caused by a single atom. atomic cluster, short range ordering etc. in a crystal should be treated more strictly. In this respect, the above equation can not be applied directly to this problem, but as a Arst approximation the maximum interaction energy, Enas., is calculated by the following equation.

$$
E_{\text {max }}=|B|_{\text {dex }} \cdot|\mu|
$$

where the magnetic moment is assumed to be parallel to the magnetic induction. Then by substituting the value of maximum induction on the radius of $5 \mathrm{~b}$ and the Bohr magneton $10^{-23} / / T e s l a$ into the above equation 4-42, the "magnetic interaction energy" $\sim 10^{-9} \mathrm{ev} / \mathrm{b}$ is obtained. This value is very much smaller than those of elastic or electric interaction energy.

The following two lacts might be noticed in the above calculations. First of all, those calculations were carried out for the infinite screening constant which means non-screening effect. As was mentioned in the previous section. IV-1, the effect is not significant, and these estimations are expected not to be seriocsly modiffed. Secondly, the values of the magnetic induction on the radius of $5 b$ were adopted for the above estimations. Within this radius, both the magnitude of induction and interaction energy are more magnifled. However, without clear knowledge of core, those values should not be allowed to come into the estimation. Thon the vaiues on the assumed core radius 5 b should be viewed as a possib! , estimation provided that from the outside core to the inside core physical values suck as electron density are smoothly changtise and, at the core radius, a maximu n value appears.

The abore discussion criticizes several unclear physics bebind this calculaHon. But, as the very rough arst estimation, one can conclude that both the 
influence of the induced magnetic feld (dyaamic effect) on the previous calculation results and the possibility of magnetic interaction with a magnetic cluster are hardly expected.

IV-6. Possibility of The Extension to Yany Body Problem (Collective Beharior of Dislocations) in Very Low Temperature Deformation

The main subject of this study bas been tne calculation of the interaction force between a single dislocation and the electrons. Most theoretical studies of very low temperature deformation hehavior also have been concentrated on the single dislocation behavior. Even creep or stress relaxation phenomena. which can be observed even in the very low temperature region and should be essentially described by group behavior of dislocations, jave been approached based on single dislocation behavior. Although, to some extent, those analyses have revealed the essence of the phenomena by int:"oducing new interactions such as the dynamic effect or quantum effect. all explanations and predietions remain qualitative arguments because of the lack of knowledge of many body eflects. The study of the interaction force between two dislocations for various type of sonflgurations is certainly the important basic problem, but it is virtually impossible to apply the result to each single dislocation constituent and to analyze the macroscopic behavior even if a huge computer is available. Introduction of a thermodynamic treatment is essential. ln this section, as one of many body problems, the possibility of obtaining the mobile dislocation density as a function of given deformation conditions such as temperature strain rate, damping constant and so on is discussed.

\section{IV-6-1. Sumino Hypothesis}

Historically, the many body problem was first discussed by Johnston and 
Gilman [42], and they clearly clarifed the dependency of the yielding behavior on various deformation factors. Haasen and Alexunder's analysis [43] is also one of the typical examples of the many body problem. These treatments can reproduce the stress-strain curve under the given deformation condition. However. empirical equations are involved in the analysis. Moreover the physics behind many body effects are not rell discussed. In this respect, one should say that these trea.nents are hybrid-theoretical treatments. Oo the other hand, Sumino et.al. $[44,15]$ recenlly proposed a potentially useful hypothesis in the following ray.

The strain rate $\dot{\varepsilon}$ was given by

$$
\dot{\varepsilon}=N_{m} b \bar{v}
$$

Mathematically, an infinit, number of combinations of $N_{m}$ and $\bar{v}$ are possible under a given strain rate $\dot{\varepsilon}$. However, this combination is not determined arbitrarily and there should be some physica: principles which dominate the best combination of $N_{m}$ and $\bar{v}$. Sumino et. al. proposed the following hypothesis to determine the enn sination. The steady state of moving dislocations is determined so as to make the component of the fow stress associated with moving dislocations the minimum necessary to maintain a given strain rate. The mathematical expression of the above hypothesis is given in the next equation.

$$
\frac{\partial\left(\tau_{u f t}+\tau_{i}\right)}{\partial N_{m}}=0
$$

where $\tau_{\text {off }}$ is the eflective stress on a dislocation and $\tau_{i}{ }^{\circ}$ is the interaction force among moving dislocations. The validity of this hypothesis has been reported to be proved for Ge and Si by their experiments [4e]. And by applying the eq. 4-43. they successfully explained the dependency of the effective stress on strain rate and derived the equilibrium moving dislocation density as a function of 
delormation conditions such as strain rate etc.. Moreover, based on irreversible thermodynamies, Nishioka [47] theoretically proved that this hypothesis is Identical with the condition of the minimum rate of entropy production associated with plastic deformation.

Because of the simplicity of its mathematical statement, Sumino's analysis provides a great possibility of extension. However, there are two diffculties which should be ogercome in its application to this study. One is the dyamic effects, ie. damping or inertia, which are not taken into account in their treatment. The cther one is the difference of the mode of a dislocation motion. Their interest was focused on Ge and Si which have higher Peierls potential, and dislocation motion is characterized by the viscous mode. But in the nase of dilute f.c.c. alloys, the Peierls potential is noc high and obstacles are scattered point-wise; then the dislocation motion is characterized as fr. Alight motion [40]. (It is not too much to say that the existence of dynamic eflects is limited to Iree flight wotion and except for some anomalies arising Irom quantum tunneling througt the high Peieris potential, most of the deformation ariomalies at very low temperature are centered on t.c.c. alloys.)

IV-B-2. Extension to Pree Flight Thermally Activated Yotion with the Aid of Dyoure Efrect

The above diffeulties are opercome by the following modeling processes. The model schematically shown in the Fig.19 is considered and the following deformation parameters are initially introduced. $N_{m n}$ is the mobile dislocation density: $b$ is the Burgers vector on this slip plane: $c$ is the concentration of point obstacles; $\tau_{\text {app }}$ is the applied stress; $\tau_{\text {off }}$ is the effective stress on a dislocation; $B$ is the damping constant and the deformation temperature is $T$. In the case of viscous dislocation motion, the strain $\Delta t$ during time $\Delta t$ was given by 


$$
\Delta \varepsilon=N_{m} \cdot \Delta \bar{X} \cdot b
$$

where $\Delta \bar{X}$ was the average displacement of mobile dislocations during time $\Delta t$. Then the strain rate $\dot{\varepsilon}$ was derivec as

$$
\begin{aligned}
E & =\lim _{\Delta t \rightarrow 0} \frac{\Delta \varepsilon}{\Delta t} \\
& =\lim _{\Delta t \rightarrow 0} N_{m} \frac{\Delta \bar{X}}{\Delta t} b \\
& =N_{m} \bar{v} b
\end{aligned}
$$

This is quite a gencral and familiar formula for the strain rate. On the other hand, in the case of tree tight motion, it should be modified in the following way. As the rate controliting process of a dislocation motion. thermally activated processes at an obstacle assisted by an inertia effect is assumed. This implies that the fight time between obstacles is negligible as compared with the captured time at an obsi the thermal activation is assumed to take place at $\Delta P$ obstacles per unit volume during time $\Delta t$. Then. the area. $A_{\text {sugst }}$.

$$
A_{\text {mops }}=\Delta P\left(L_{n}\right)^{2}
$$

is swept away by dislocations and the strain, $\Delta \varepsilon$, per unit volume is given by

$$
\Delta \varepsilon=\Lambda P \cdot\left(L_{3}\right)^{2} b
$$

therefore strain rate is derived as

$$
\begin{aligned}
\dot{\epsilon} & =\lim _{\Delta t \rightarrow 0} \frac{\Delta \varepsilon}{\Delta t} \\
& =\lim _{\Delta t \rightarrow 0} \frac{\Delta P}{\Delta t}\left(L_{n}\right)^{2} b
\end{aligned}
$$




$$
=\dot{P}^{+}\left(L_{3}\right)^{2} b
$$

where $L_{l}$ is the average distance among obstacles, and $\left(L_{s}\right)^{2}$ is the average area occupied by an obstacle. The most important assumption implied in eq. 4-46 is tbat, after overcoming an obstacie, a dislocation should be captured by the next obstacle it encounters. This assumption was originally made by Friedel [50] in his study of static dislocations and has been succeeded to following studies.

Let $\bar{L}$ be the average distance between obstacles along dislocations. Then the number of total points (obstacles) interacting with a dislocation is given by

$$
P_{r}=\frac{N_{m}}{\bar{L}}
$$

Accordies tu ine thermal activation process developed by Suzuki [51] the waiting (captured) time at an obstacle, $t_{w}$, is

$$
\frac{l}{t_{V}}=\nu \exp \left(-\frac{G_{0}-b d \bar{L} T_{\text {off }} \overline{Y_{m}}}{k T}\right)
$$

where $\nu$ is the trial frequency. $G_{0}$ is the interaction energy between an obstacle and a dislocation, $d$ is the width of an obstacle, and $y_{m}$ is the parameter specifles the dynamic effect which is discussed later. The numerator of the exponent in the eq. $4-51$ is the activation energy. Since $\dot{P}^{+}$in the eq. $4-49$ should be given by

$$
\dot{P}^{+}=\frac{P_{T}}{t_{T}}
$$

then substitution of eqs. 4-50 and 4-51 into the eq. 4-49 yields

$$
\dot{c}=\left(\frac{N_{m}}{\bar{L}}\right) \nu \exp \left(-\frac{G_{0}-b d \bar{L} \tau_{U / P} \bar{T}_{m}}{k T}\right)=\dot{z}_{b}
$$


This is the anal expression of the strain rate extended to the free flight mode of a dislocation motion.

The physical meaning of $Y_{m}$ is diseussed. As is shown in the Fig.20, the components of the line tension in the direction of dislocation motion, $F_{m}$, was given by

$$
\begin{aligned}
& F_{m}=b \tau_{m f t} \overline{L Y_{m m}} \\
& \overline{Y_{m}}=1+0.6 \mathrm{e}^{-\mathrm{m}^{\circ}}
\end{aligned}
$$

and

$$
x^{*}=\pi \gamma^{*} / \omega_{0}
$$

where $\gamma^{*}$ and $\omega_{0}$ were

$$
\gamma^{*}=\frac{B}{2 m}
$$

and

$$
\omega_{0}=\pi\left(\frac{\Gamma}{m_{d}}\right)^{1 / 2}, \bar{L}
$$

These quantities can be derived as the solution of the dislocation motion equation $1-1$ of a string model. And $Y_{m}$ which is multiplied by the static equilibrium foree, $b T_{\text {off }} \bar{L}$, can be viewed as the modiflcation tactor which specifes the dynamic effect. Since the vibration of dislocaticn is repeated many times, the dynamic effect due to the vibration should be incorporated to thermal activation process and the average value during the decay time $\frac{1}{\gamma^{\circ}}$ is inserted to the equation. $Y_{m}$ in the eq. 4-54 is such an average $T$ due calculated by the following equation. 


$$
\begin{aligned}
\overline{Y_{m}} & =1+\frac{e^{-z^{*}}}{1 / \gamma^{*}} \int_{0}^{\frac{1}{\gamma^{*}}} e^{\rightarrow t^{*} t} d t \\
& \cong 1+0.6 e^{-z^{*}}
\end{aligned}
$$

This elaborate idea devised by Suzuki [12] seems to allow coexistence of the dynamic effect and the thermal activation process. However, thermal activation is essentially a statistical fluctuation phenomena, the deterministic dynamic term can not be accommodated in such a manner. The basic solution of this extremely hard problem might be settled by returning to basic physics [52]. In this study. Suzuki's method is adopted.

The average distance between obstacles along dislocations, $\bar{L}$, is generally a function of stress, line tension etc. and the search for the proper functional form belongs to the statistical problem of hardening. In the case of a static dislocation this problem has been almost worked out $[53,64]$. However, to the author's knowledge, nobody has succeeded in obtaining such a function for the general dynamic problem. Instead of going deeply into this problem. Friedel's statistics is applied under suitable assumptions. The traditional Friedel statistics tells that $\bar{L}$ can be related with stress, line tension and concentration of obstacie by the folloring equation.

$$
\bar{L}=\left(2 \frac{\Gamma}{b c}\right)^{\frac{1}{3}} \tau^{-\frac{1}{3}}
$$

This relation was derived trom the static force balance condition at an infinitesimal portion of a bowng arc and geometrical condition on the assumption that the area which is swept out by a dislocation between two obstacles is equal to the average area occupied by an obstacle. It should be noticed that 
application of the Friedel's statistics immediately confine this problem to restricted condition: the vibration amplitude of a bowing string should not be very much deviated trom static equilibrium configuration and, once overcoming an obstacle, a dislocation loses all memories about its dynamic vibration. In other words, the current overcoming process should not be influeaced by previous processes. Moreover, unzipping effects can not be included. Substitution of the eq. 4-60 into the eq. 4-53 immediately yields

$$
i=N_{m} \nu\left(\frac{b^{4}}{2 \Gamma c^{2}}\right)^{\frac{1}{3}} T_{j \rho f}^{\frac{1}{5}} \cdot \exp \left(-\frac{G_{0}-b d\left(\frac{2 \Gamma}{b c}\right)^{\frac{1}{5} s} T^{\frac{2}{3}} \frac{Y_{m}}{k T}}{k T}\right)
$$

and therelore

$$
\mathrm{Q}=\ln \tau_{0 / f}+\phi\left(1+0.6 e^{-9}\right) \tau_{0}^{\frac{2}{3}}
$$

where

$$
n=3\left\{\ln \frac{\dot{\varepsilon}}{N_{m} v\left(\frac{b^{4}}{2 \Gamma c^{2}}\right)^{\frac{1}{s}}}+\frac{G_{0}}{k t}\right\}, \Phi=\frac{3 b d}{k T}\left(\frac{2 \Gamma}{b c}\right)^{\frac{1}{5}}
$$

The dnal stage is the application of Sumino hypothesis to eq. 4-62. By assuming a suitable form of the interaction force between moving dislocations and the work hardening force, one would be able to describe the effective stress more concretely.

$$
\begin{aligned}
T_{o j ;} & =\tau_{\text {app }}-T_{i}^{i}-T_{h} \\
& =T_{\text {app }}-A^{*} \sqrt{N_{m}}-2\left(\varepsilon-\varepsilon_{0}\right)
\end{aligned}
$$

where $\tau_{i}^{0}$ is the interaction force among moving dislocation and $\tau_{\Lambda}$ is caused by 
static dislocations, namely the work hardening effect. But eqs. 4-83 and 4-84 are nothing more than assumptions. To obtain proper functional forms sbould be recognized as another important aspect of the many body problem. It will require very hard mathematical load to separate out $\tau_{\text {ofs }}$ Irom the eq. 4-62 and it may not be able to be performed analytically. But by substituting eq. $4-64$ into such a expression followed by an application of eq. 4-43, one will obtain the equilibrium (steady state) moving dislocation density, $N_{m}^{\bullet}$. as a lunction of deformation condition. By substituting the electric damping constant obtained in the previous chapter eqs.3-90 and 3-91 into the. $N_{m}^{*}$, one can discuss the low temperature deformation phenomena more reliably as a further unifled treatment. Those are possibilities and for future consideration. 


\section{v. CONCLUSTONS}

As stated in the introduction, the primary roal of this study was to calculate the electron damping force in a normal state for a moving edge dislocation. Based on the free electron gas model, an equation which describes the electron friction force on a dislocation was analytically derived as a function of the normalized velocity of a dislocation and the Fermi vector. Damping constants whlch are determined from the force-velocity relations are generally a function of velocity. However, in the range of physically meaningful dislocation velocities, the force is lineariy related to the velocity. Theoretical values of the damping constant obtained in this study shows fairiy good agreenents with experimental values. Electrical resistivity, as a middle product of this study, was compared with an experimental value lor pure Al. The discrepancy is about an order of two. This discrepancy was ascribed to DMR sources of the sample. Also, direct numerical calculation was carried out to estimate the magnetic aeld associated with a moving dislocation. And the possibility of a magnetic interaction between a moving dislocation and local magnotic moments in a crystal was evaluated. The calculated magnetic fleld was negligibly small and any magnetic effects were hardly expected. Although ambiguity of core structure remains in the model, as a whole, this model provides farly good estimations.

A possibility for a unifed treatment of the three main dislocation problems, elementary interaction manner, statistical problem of hardening and collective behavtor of Jlslocations, was discussed in the tnal section. And a fundamental equation, rhich may describe the essential features of deformation phenomena at very low temperatures, was derived. To solve the equation and to oxtead the model remaln for fucure work. 
Appendix A. Born-Approximation and Calenietion of Seattering (Transition) Probability $\langle 1\rangle$

The time dependent Schrödinger equation can be given hy

$$
i \pi \frac{\partial \Psi^{0}}{\partial t}=H^{0} \Psi^{0}
$$

where $\psi^{\circ}$ and $H^{\circ}$ are the wave function and unperturbed Hamiltonian. The solution r.f above equation is easily found to be

$$
\Psi_{n}^{0}=\psi_{n} \exp \left(i \frac{E_{n} t}{n}\right)
$$

where $\psi_{n}$ is spave part of the wave function. Now, let us consider the perturbed system with perturbation potential, $\Delta U$. The corresponding Schrōdinger equation is given by

$$
i n \frac{\partial \Psi}{\partial t}=\left(H^{0}+\Delta U\right) \Psi
$$

The series expansion of $\Psi(k, t)$ with respect to unperturbed wave function, $\Psi_{n}^{\circ}$ gives

$$
\Psi(\mathbf{k}, t)=\sum_{n} \dot{a}_{n} \Psi_{n}^{0}(\mathbf{k}, t)
$$

a -an be obtained by substituting $(A-4)$ into $(A-3)$.

$$
i \pi \sum_{n} \frac{d a_{n}(t)}{d t} \Psi g(k, t)=\Delta U \Psi(k, t)
$$

By multiplying the complex conjugate of $\psi_{m}^{0}, \Psi_{n_{*}}^{\circ}$, to the both sides of above equation, the tollowing equation is obtained.

$$
i \pi \frac{d a_{n}(t)}{d t} \delta_{m n}=\int_{k} \Psi_{m}^{00} \Delta U \Psi d r=i \pi \frac{d a_{n}}{d t}
$$


Here. the perturbation potential is assumed to be wak so that $\Psi(\mathbf{k}, t)$ in the eq. $(A-6)$ can be approximated to be $\Psi^{0}(k, t)$ (Born Approximation). Then eq. (A-6) becomes

$$
i \kappa \frac{d a_{n}}{d t}=\int_{k} \Psi_{n}^{0}(k, t) \Delta U \Psi^{0}(k, t) d k
$$

In stead of the integral notation of the above equation, the folloring compact notation is introduced.

$$
\begin{aligned}
U_{n 0} & =\int \Psi_{n}^{0}(k, t) \Delta U \Psi^{0}(k, t) d k \\
& =\left\langle\Psi_{n}, \Delta U \mid \Psi^{0}\right\rangle \\
& =\langle n|\Delta U| 0\rangle \cdot \exp \left(\frac{E_{0}-E_{n}}{\pi} t\right)
\end{aligned}
$$

By using above notation, the solution of eq. (A-7) can be given by

$$
a_{n}=\frac{1}{i \hbar} \int_{0}^{t} U_{n 0} \cdot \exp \left[i \frac{E_{0}-E_{n}}{\hbar} t\right] d t
$$

Since the oerturbation potential. $\Delta U$, of the present study is not time dependent, $(A-9)$ can be further more simplified and

$$
\begin{aligned}
a_{n} & =\frac{U_{n 0}}{i \pi} \int_{0}^{l} \exp \left[i \frac{E_{0}-E_{n}}{\pi} t\right] d t \\
& =-\frac{U_{n 0}}{\Delta E}\left\{e^{i \frac{\Delta \varepsilon_{t}}{\pi}}-1\right\}
\end{aligned}
$$

where

$$
\Delta E=E_{0}-E_{n}
$$

At time $t$, the probabllity, $P_{n}$, of anding the system at state, $n$, is given by 


$$
P_{n}=\left|a_{n}(t)\right|^{2}
$$

Then the substitution of $(A-10)$ into $(A-11)$ yields

$$
\begin{aligned}
P_{n} & =\left|a_{n}(t)\right|^{2} \\
& =\frac{\pi t}{\hbar^{2}}\left(U_{n 0}\right)^{2} \frac{\sin ^{2} \frac{\Delta E}{2 \hbar} t}{\pi t(\Delta E / 2 n)^{2}}
\end{aligned}
$$

The third term of (A-12) is known as Fejer kernel [55] which provides the following $\delta$-sequence.

$$
\frac{\sin ^{2} \frac{\Delta E}{2 \hbar} t}{\pi r^{2}(\Delta E / 2 \hbar)^{2}} \sim \delta\left(\frac{\Delta E}{2 \pi}\right)
$$

Therelore the scattering probability per unit tirne, $Q(0, a)$, can be given by

$$
\begin{aligned}
Q(0, n) & =\frac{P_{n}}{t} \\
& =\frac{\pi}{\pi^{2}}|\langle n|\Delta U| 0\rangle|^{2} \delta\left(\frac{\Delta E}{2 \pi}\right)
\end{aligned}
$$

Appendix B. Calculation of Scattering Probability $\langle\Pi\rangle$ - Fourier Tranglormation of Perturbation Potential -

Scattering probability matrix. $S_{k \neq,}$, was given by

$$
S_{k+k}=\langle\mathbf{k}|\Delta U| \mathbf{k}\rangle
$$

16. was tree electron wave function and is explicitly expressed as [56]

$$
|k|=\frac{e^{i k r}}{\Delta^{1 / 2}(2 G+1)^{3 / 2}}
$$


where $\Delta$ is the volume of the unit cell and $(2 G+1)^{\mathrm{s}}$ is the number of atoms in the crystal. Since constant term of $\Delta U$ can be separated as

$$
\begin{aligned}
\Delta U & =-e \varphi(\mathbf{r}) \\
& =-\frac{D}{a} B C V_{0}
\end{aligned}
$$

where

$$
U_{a}=\frac{\gamma y}{x^{2}+(\gamma y)^{2}}-2 \sqrt{a} K_{2}(\sqrt{a}|r|) \frac{\sin \theta}{\gamma+1}
$$

attention is conflned to the Fourier transformation of $U g$. Let $T$ and $S$ be the Fourier transformation of the first term and the second term, respectively. Then,

$$
\begin{aligned}
& T=\int_{-\infty}^{\infty} \int_{-\infty}^{-i x} \frac{x y}{x^{2}+(x y)^{2}} d x d y
\end{aligned}
$$

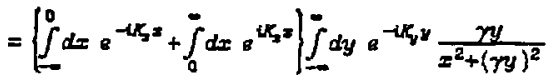

$$
\begin{aligned}
& =\int_{0}^{\infty} d x e^{-i\left(-T_{1}\right) x} T_{1}(x)+\int_{0}^{\infty} d x e^{-x \xi_{1} x} T_{1}(x)
\end{aligned}
$$

where

$$
\mathbf{x}=\mathbf{k}-\mathbf{k}
$$

and

$$
T_{1}(x)=\int_{-\infty}^{\infty} d y e^{-2 k, y} \frac{y y}{x^{2}+(y y)^{2}}
$$

By putting $r y$ to $Y$, above equation is transformed to 


$$
T_{1}(x)=\frac{1}{\gamma} \int_{-\infty}^{\infty} d Y \varepsilon^{-4\left|\frac{x_{1}}{\gamma}\right| Y} \frac{Y}{x^{2}+Y^{2}}
$$

Ey the say according to Campbell [57],

$$
\int_{-\infty}^{\infty} \frac{p}{p^{2}-\beta^{2}} e^{i 2 \pi \rho g} d f= \pm \frac{1}{2} e^{-\rho|g|}
$$

where

$$
p=2 \pi i f . \beta>0 \text { and } \pm g>0
$$

Then comparison of eq. (B-7) with eq. $(B-8)$ immediately gives

$$
\begin{aligned}
T_{1}(x) & =\frac{1}{\gamma}\left( \pm \pi i e^{-2 \pi x\left|-\frac{1}{2 \pi} \frac{K_{x}}{\gamma}\right|}\right) \\
& = \pm \frac{\pi i}{\gamma} e^{-\frac{x}{\gamma}\left|K_{y}\right|} \quad\left(0> \pm K_{y}\right)
\end{aligned}
$$

and substitution of the eq. $(B-10)$ into the eq. (B-4) yields

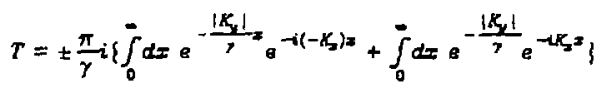

By making use of the No. 438 of the same relerence [57].

$$
\begin{aligned}
\int_{-}^{-12 \pi f \alpha} a^{-\beta g} d g & =\frac{1}{p+\beta} \\
& =\frac{1}{i 2 \pi f+g} \quad(g>0)
\end{aligned}
$$

comparison of eq. (B-11) with eq. (B-12) yields the following result.

$$
T= \pm 2 \pi i \frac{\left|K_{y}\right|}{\left(\gamma K_{z}\right)^{2}+K_{y}^{2}} \quad 0> \pm K_{y}
$$


On the other hand, $\mathrm{S}$ is given by

$$
\begin{aligned}
& S=\int_{-\infty}^{\infty} \int_{-\infty}^{-1 \pi} \sqrt{a} K_{1}(\sqrt{a}|r|) \frac{\sin \vartheta}{\gamma+1}
\end{aligned}
$$

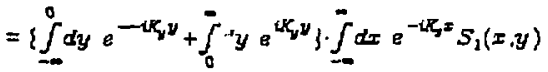

$$
\begin{aligned}
& =\left\{-\int_{0} d y e^{-i\left(-x_{y}\right) y}+\bar{\int}_{0} d y e^{-i x_{y} y}\right\} \cdot \bar{\int}_{-} d x e^{-i x_{2} x} S_{1}(x, y)
\end{aligned}
$$

where coefficient 2 in the eq. $(B-3)$ is neglected and $S_{1}(x, y)$ is given by

$$
\begin{aligned}
S_{1}(x, y) & =\sqrt{a} K_{1}(\sqrt{a}|r|) \frac{\sin y}{\gamma+1} \\
& =\frac{1}{\gamma+1} \frac{\sqrt{a} y}{\sqrt{x^{2}+y^{2}}} K_{1}\left(\sqrt{a} \sqrt{x^{2}+y^{2}}\right)
\end{aligned}
$$

According to No. 867 of the reference [57].

$$
\int_{-} d g \frac{\alpha \rho K_{1}\left[\rho\left(g^{2}+a^{2}\right)^{1 / 2}\right]}{\pi\left(g^{2}+a^{2}\right)^{1 / 2}} e^{-12 \pi f g}=\exp \left[-\alpha\left(\rho^{2}-p^{2}\right)^{1 / 2}\right]
$$

where

$$
p=2 \pi i f, p z \text { and } a>0
$$

Direct comparison between the last term of eq. (B-14) and eq. (B-16) leads the following Fourier transformation of $S_{1}(x, y)$.

$$
\int_{-\infty}^{-r} d x e^{-x_{2} x} S_{1}(x, y)=\frac{\pi}{\gamma+1} e^{-y} \sqrt{a+R_{5}^{2}}
$$

Then, $\mathrm{S}$ becomes

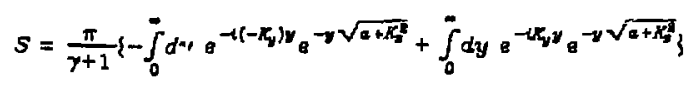


Again by making use of the Ne, $43 B$ of the reference [57] given by (B-12), Fourier transtormation of each term is immediately calculated.

$$
\begin{aligned}
S & =\frac{1}{\gamma+1}\left(-\pi \frac{1}{-i K_{y}+\sqrt{a+K_{z}^{2}}}+\pi \frac{1}{i K_{y}+\sqrt{a+K_{z}^{2}}}\right) \\
& =\frac{1}{\gamma+1} \frac{(-i) 2 \pi K_{y}}{K_{x}^{2}+K_{y}^{2}+a}
\end{aligned}
$$

The final form of scattering probability matrix is given by

$$
S_{k E}= \pm 2 \pi i \frac{\left|K_{y}\right|}{\left(\gamma K_{z}\right)^{2}+K_{\gamma}^{2}}+\frac{4 \pi i}{\gamma+1} \frac{K_{y}}{K_{\pi}^{2}+K_{z}^{2}+a}
$$

Appendix C. Numerical Evaluation of Dislocation Resistivity at $0^{\circ} \mathrm{K}$

\section{C-1. Mathematical Basis}

Mathematical basis' of the numerical evaluation for a single integral equauon of Fredholm type is provided in the reference [31]. Here, essential points are summarized. The following Fredholm inhomogeneous integral equation is considered.

$$
\int_{a}^{k} k(x, \xi) u(\xi) d \xi-\mu u(z)=f(x)
$$

In urder to solve above equation numerically, interval $[a, b]$ is divided into $n$ equal steps, and integral is replaced by summation.

$$
\int_{\varepsilon}^{b} k(x, \xi) u(\xi) d \xi=\sum_{j=i}^{n} k\left(x, x_{j}\right) u\left(x_{j}\right) \frac{b-a}{n}
$$

The integral equation (C-1) is attempted to be satistied only at $x_{1}, x_{2} \ldots \ldots x_{n}$. By introducing the following notations 


$$
\begin{aligned}
& k\left(x_{i}, x_{j}\right)=k_{i j} \\
& u\left(x_{i}\right)=u_{i}
\end{aligned}
$$

and

$$
f\left(x_{1}\right)=f_{i}
$$

eq. $(C-1)$ is transformed to $\pi$ sets of linear algebraic equations.

$$
\sum_{j} k_{i j}-u_{j} \frac{b-a}{n}=\mu u_{i}+f_{i}
$$

After solving these $n$ linear inhomogeneous equations for unknown $u_{1}, u_{2}, \ldots \ldots$, $\boldsymbol{u} \boldsymbol{x}$, a functional representation of $\boldsymbol{u}(\boldsymbol{x})$ can be obtained as

$$
u(x)=-\frac{f(x)}{k i}+\frac{1}{\mu} \sum_{j=1}^{n} k\left(x, x_{y}\right) u_{j} \frac{b-a}{\pi}
$$

c-2. Numerical Calculation of Resistivity

By applying the following relation $(C-8)$ to the eq. 4-18

$$
\begin{aligned}
\boldsymbol{E} & =\left(g_{x}, g_{\mathrm{y}}, g_{\mathrm{a}}\right) \\
& =\left(h_{\mathrm{z}} E_{\mathrm{x}}, h_{y} E_{\mathrm{y}}, h_{\mathrm{z}} E_{\mathrm{z}}\right)
\end{aligned}
$$

the B.T.E. can be split into three equations for $x, y$ and $z$ ccm? snents.

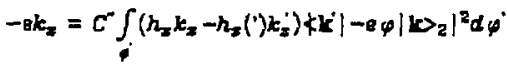

$$
\begin{aligned}
& \text { etc. }
\end{aligned}
$$

etc. Since procedures are same for each component, this argument is conflned to only :: component. By performing the coordinate transformation given by the eqs. $3-44 \sim 3-48$, above eq. (C-9) can be revritten as 


$$
-\varepsilon \cos \varphi=C^{\prime \prime} \int\left(h_{x} \cos \varphi-h_{z}\left({ }^{\prime}\right) \cos \varphi^{\prime}\right) F^{\prime}(\vartheta, \varphi, \varphi) d \varphi^{\circ}
$$

Where $F^{*}\left(\theta, \varphi, \varphi^{\circ}\right)$ is the polar coordinate representation of scattering probability matrix $\left|S_{k j}\right|^{2}$ given by (B-21). On the other hand, by using (C-8), the conductivity, $\sigma_{z x}$, can be singled out from the eq. 3-58.

$$
\sigma_{x x}=A k_{F} \int_{0}^{\pi} d v \int_{0}^{2 \pi} d \varphi h_{x}(\vartheta, \varphi) \sin ^{3} \vartheta d \varphi
$$

where $A$ was given in the eq. 3-61. Above double integral can be replaced by summation is the following ray.

$$
\begin{aligned}
& \sigma_{z=}=A k_{p} \sum_{i=1}^{\psi_{i} 1} \sum_{j=1}^{2 \mu} h_{j}\left(v_{i}, \varphi_{j}\right) \cdot \sin ^{3} v_{i} \cdot \cos ^{2} \varphi_{j} \frac{\pi}{H} \frac{2 \pi}{2 M} \\
& =A t_{P} \sum_{i} \sum_{j} h_{i j} \cdot \sin ^{3} v_{i} \cdot \cos ^{2} \varphi_{j}\left(\frac{\pi}{N}\right)^{2}
\end{aligned}
$$

where compact notation $h_{(j}$ is introduced for $h_{f}\left(\vartheta_{1}, \varphi_{j}\right)$. And substitution of $h_{\text {, }}$ the solution of $(C-10)$, into the eq. (C-12) yields numerical value of conductivity. This is the essential procedure.

From $F^{\prime \prime}\left(v, \varphi, \varphi^{\prime}\right)$, one can single out $1 / \sin ^{2} \approx$ term. Then $(c-10)$ is slightly moditled to be

$$
-\theta \sin ^{2} \psi \cdot \cos \varphi=C^{\prime \prime} \int_{i^{\prime}}\left(h_{x} \cos \varphi-h_{3}\left({ }^{\prime}\right) \cos \varphi^{\circ}\right) F\left(\vartheta, \varphi, \varphi^{\circ}\right) d \varphi^{\prime}
$$

On the $\vartheta_{1}-\varphi_{k}$ space, above eq. $(C-13)$ can be rewritten as

$$
\begin{aligned}
& -e S_{i} \cos \varphi_{k}=C^{-} \int_{0}^{2 \pi}\left(h_{i k} \cos \varphi_{k}-h\left(1^{\prime}\right) \cos \varphi^{\prime}\right) r\left(\vartheta_{i}, \varphi_{k}, \varphi^{\prime}\right) d \varphi^{\prime} \\
& (i=1,2, \ldots, H+1 ; k=2,2, \ldots, 2 M+1)
\end{aligned}
$$

where 


$$
S_{1}=\sin ^{2} \vartheta_{i}
$$

By applying the Trapezoidal rule to the eq. (C-14), integral can be replace by summation in the following form

$$
\begin{aligned}
& -e S_{i} \cos \varphi_{k}=C^{*}\left(\sum_{j=2}^{2 \mu}\left(h_{i k} \cos \varphi_{k}-h_{i j} \cos \varphi_{j}\right) P_{i}^{i} \frac{2 \pi}{2 M}\right. \\
& +\frac{1}{2}\left(h_{i k} \cos \varphi_{k}-h_{i 1} \cos \varphi_{1}\right) F_{i k}^{1} \frac{2 \pi}{2 M} \\
& \left.+\frac{1}{2}\left(h_{k} \cos \varphi_{k}-h_{i} 2 \nu+1 \cos \varphi_{2 y+1}\right) F_{k t}^{2 \mu+1} \frac{2 \pi}{2 \alpha}\right\} \\
& (i=1,2, \ldots, \ldots, H+1 ; k, j=1,2, \ldots, 2 M+1)
\end{aligned}
$$

where $F i$ is the compact notatio. $f F\left(v_{i}, \varphi_{k}, \varphi_{j}\right)$. Then, for each $(i, k), 2 U+1$ sets of linear algebraic equations for unknown hy is set up. Now, let us define new tensor, $R_{i j}$.

$$
H_{i j}=h_{v j} \cos \varphi_{j}
$$

Then eq. $(C-16)$ is transtormed to

$$
\sum_{j=2}^{2 N}\left(H_{i j}-H_{i k}\right) F_{i k}^{i}+\frac{1}{2}\left(H_{i 1}-H_{i k}\right) F_{i k}^{l}+\frac{1}{2}\left(H_{i} 2 H_{+1}-H_{i k}\right) F_{i k}^{2 K+1}=\frac{\theta}{C^{\prime}} S_{i} \cos \varphi_{k}
$$

By making use of the next relations $(C-19) \sim(C-21)$ which are easily verifled,

$$
\begin{aligned}
& h_{11}=h_{12 N+1} \\
& F_{1}=F_{2 N+1} \\
& F_{i 1}^{1}=F_{i 2}^{2} 1 .
\end{aligned}
$$

$2 M+1$ sets of equations can be reduced to $2 M$ sets of equations. And the following matrix notation of $(C-18)$ is introduced. 


\begin{tabular}{|c|c|c|c|c|}
\hline$-\left(F_{i 1}^{2}+F_{i 1}^{3}+\ldots . .+F_{i 1}^{2 H}\right)$ & $F_{i 1}^{2}$ & $F_{\mathrm{i}}^{3}$ & . & $F_{i}^{2 L}$ \\
\hline$F_{t 2}^{1}$ & $-\left(F_{i 2}^{1}+F_{i 2}^{*}+\ldots . .+F_{i 2}^{u}\right)$ & $F_{i 2}^{g}$ & . & $F_{i 2}^{2 y}$ \\
\hline$\cdot$ & $\cdot$ & $-\left(F_{i b}^{1}+F_{i b}^{2}+F_{i b}^{4}+\ldots+F_{i s}^{2}\right)$ & $\cdots$ & 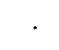 \\
\hline · & . & . & $\cdots$ & - \\
\hline$F_{i 2 x}^{i}$ & $F_{i 2 N}^{\dot{z}}$ & $\dot{F}_{i 2 y}^{\dot{2}}$ & $\begin{array}{l}\cdots \\
\cdots\end{array}$ & $i_{2 y+}^{2}$ \\
\hline
\end{tabular}

$$
\begin{array}{r}
{\left[\begin{array}{c}
H_{i 1} \\
H_{i 2} \\
\vdots \\
H_{i 2 X}^{\prime}
\end{array}\right]} \\
=\frac{\theta}{C} S_{4}\left[\begin{array}{c}
\cos \varphi_{1} \\
\cos \varphi_{2} \\
\vdots \\
\cos \varphi_{2 K}
\end{array}\right]
\end{array}
$$

Then for each $v_{i}, 2 M$ sets of $H_{i t}$ value, i.e. $h_{t}$, is numerically evaluated. This procedure is repeated $M$ times for $t_{1}, v_{2}, \ldots \ldots, t_{1}, \ldots \ldots, v_{y}$, and by substituting the results into the eq. $(C-12)$, one can obtain conductivity $\sigma_{z x}$, and therefore, dislocation resistivty $p_{x}$

$$
\begin{aligned}
& \rho_{x}^{(\operatorname{man})}=\frac{1}{\sigma\left(\frac{\pi}{m}\right)} \\
& =\frac{1}{A k_{F}} \frac{1}{\sum_{i} \sum_{j} h_{\psi_{j}} \sin ^{3} \mho_{i} \cdot \cos ^{2} \varphi_{j}\left(\frac{\pi}{M}\right)^{2}}
\end{aligned}
$$

\section{Appendix D. Yodifieation for a Poly Crystal}

Although the final product of the modification of electrical resistivity for a poly crystal is given in the reference [56], the derivation is not provided. In this appendix, the gen ral derivation of the average resistivity of a poly crystal is carried out. Components of the resistivity tensor, $n_{k t}$, and unit vector $\boldsymbol{n}_{i}$ in a 
certain direction shown in the Fig.21 are given by

$$
\left[\Omega_{k i}\right]=\left[\begin{array}{ccc}
\rho_{x} & 0 & 0 \\
0 & \rho_{y} & 0 \\
0 & 0 & 0
\end{array}\right]
$$

and

$$
\left\{n_{y}\right\}=\left(\begin{array}{c}
\sin \alpha \cdot \cos \beta \\
\sin \alpha \cdot \sin \beta \\
\cos \alpha
\end{array}\right)
$$

Then the components of the resistivity, $\rho_{i}^{p s}$, in the $x, y$ and $z$ direction is obtained as

$$
\begin{aligned}
\rho_{t}^{\beta} & =n_{t-t} \eta_{1} \\
& =\left(\begin{array}{c}
\rho_{x} \cdot \sin \alpha \cdot \cos \beta \\
\rho_{y} \cdot \sin \alpha \cdot \sin \beta \\
0
\end{array}\right)
\end{aligned}
$$

Since the measurement is carried out on a normal plane to $\vec{t}$, the component of resistivity along it should be calculated.

$$
\begin{aligned}
\rho_{n}^{a f} & =\rho_{k}^{a \rho_{n_{k}}} \\
& =\sin ^{2} a\left(\rho_{z} \cos ^{2} a+\rho_{y} \sin ^{2} \theta\right)
\end{aligned}
$$

By considering the lact that the solid angle at $(\alpha, \beta)$ is given by

$$
d \omega=\sin \alpha \cdot d \alpha \cdot d \beta
$$

the average resistivity is calculated in the following integral.

$$
\int_{0}^{\pi / 2} d \alpha \int_{0}^{2 \pi} d \beta \frac{d \omega}{2 \pi} \rho_{\alpha \beta}=\frac{1}{2} \int_{0}^{\pi / 2} d ! \alpha \int_{0}^{2 \pi} d \beta \rho_{\pi}^{\alpha \beta}
$$


(9- 1$)$

$$
=0 \frac{E}{7}=
$$

$$
\left(n^{\prime}+{ }^{*} \sigma^{\prime}\right) \frac{\varepsilon}{l}=
$$




\section{FIGURE CAPTIONS}

Figure 1. Definition of two types of coordinate systems for the description of edge dislocation rnotion.

Figure 2. Numerical evaluation of the third term appearing in eq. 3-16 and comparisous of its magnitude with the other two terms. Comparisans are carried out alorg the diagonal direction in a coordinate. $v=0.25 v_{l}$ and $\sqrt{a} 1.5 \times 10^{\theta}\left[\mathrm{cm}^{-1}\right\}$

Figure 3. Same as Figure 2, but employed velocity is $v=0.5 u_{l}$ and $\sqrt{a}=1.5 \times 10^{6}$ $\left[\mathrm{cm}^{-1}\right]$

Figure 4. Same as Figures 2 and 3, but comparisons are carried out al sag a circle of radius, $\mathbf{3 b}$.

Figure 5. Configurational relations between Cartesian and Polar coordinates in k-space.

Figure 6. A model crystia, 'n which an edge dislocation motion with constant veloclty is characterized.

Figurn 7. Deviations of electron densities from those in a dislocation Iree state. contributions of the Arst term in the eq. 4-10 are compared in terms of dislocation velocity. GM and SCR stands for $\gamma=\frac{v}{v_{1}}$ and $\sqrt{\alpha^{2}}$, respectively. The darker colors indicate higher electron densities. The first term is independent of the screening constant.

Figure 8 . Contributions of the second term and whole term in eq. 4-10 are compared in terms of dislocation velocity for a constant screening coostant, $\sqrt{a}=1.0$.

Figure 9. Comparisons for a constant screening constant, $\sqrt{a}=1.5$.

Figure 10. Comparisons for a constant screening constant, $\sqrt{a^{2}}=2.0$. 
Figure 11. Schematic picture of the employed perturbation potential eq. 3-18 for the case of $\frac{v}{v_{l}}=0.5$ and $\sqrt{a}=1.0 \times 10^{8}\left[\mathrm{~cm}^{-1}\right]$

Figure 12. Contribution of the first term in the eq. 3-18.

Figure 13. Contributions of the second term, the screening term

Figure 14. Dependencies of the force tactor $F^{*}$ in the eq. 3-90 on the normalized velocities for four kinds of Fermi vector. unit is arbitrary.

Figure 15. Magniled graph of Figure 14 for normalized velocities less than 0.01. Unit is same as Figure 14.

Figure 16. Dependencies of Resistivities on normalized velocities for nine kinds of Fermi vector.

Figure 17. Geometrical distribution of magnetic induction calculated numerically tor the case of $\frac{v}{v_{l}}=0.3$. Values in the figure are normalized by constant terms in the eq. 3-98.

Figure 18. Dependencies of magnetic induction on $y$-coordinates at fixed $x$ coordinate, $x=5 b$ for three types of velocities.

Figure i9. A model which describes collective behavior of dislocations.

Figure 20. Magnified view of force balance condition on a dislocation interacting with point obstacles.

Figure 21, Relations among coordinates appeaning in APPENDIX D. 


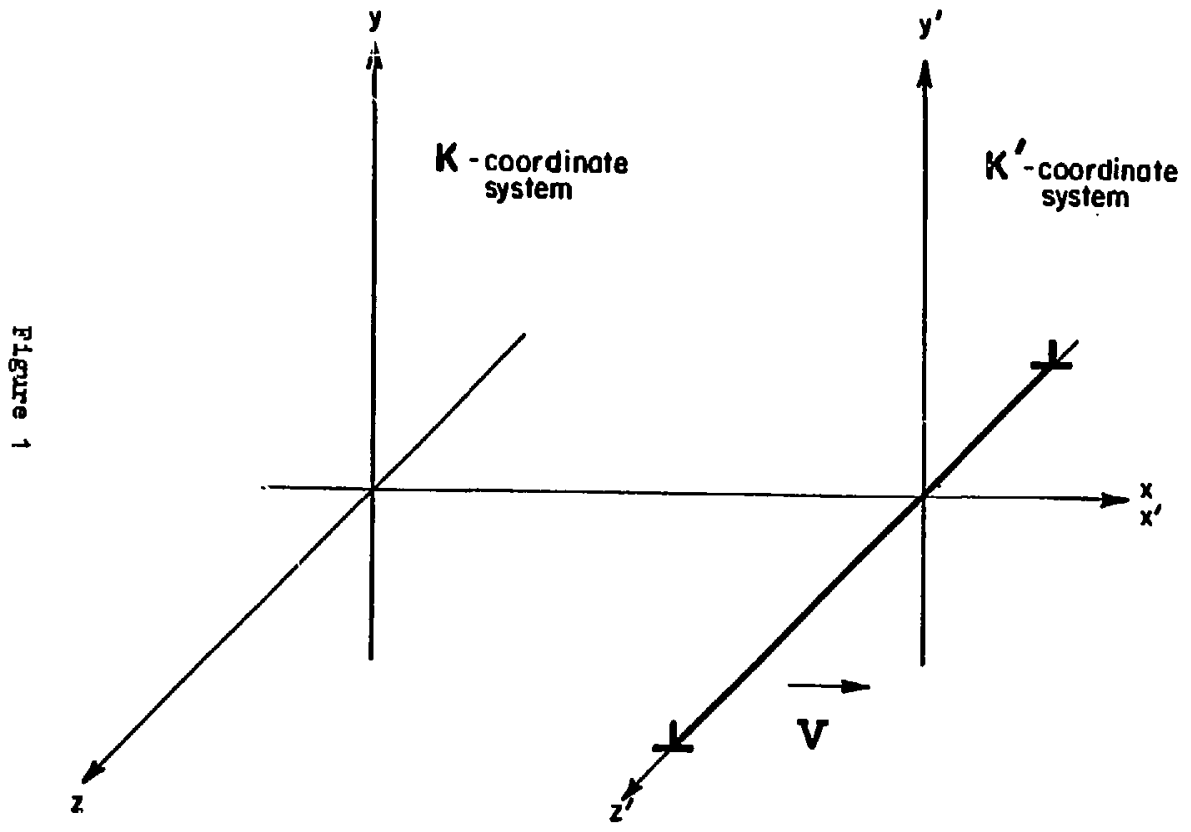

ฌ

XBL82II-6805 


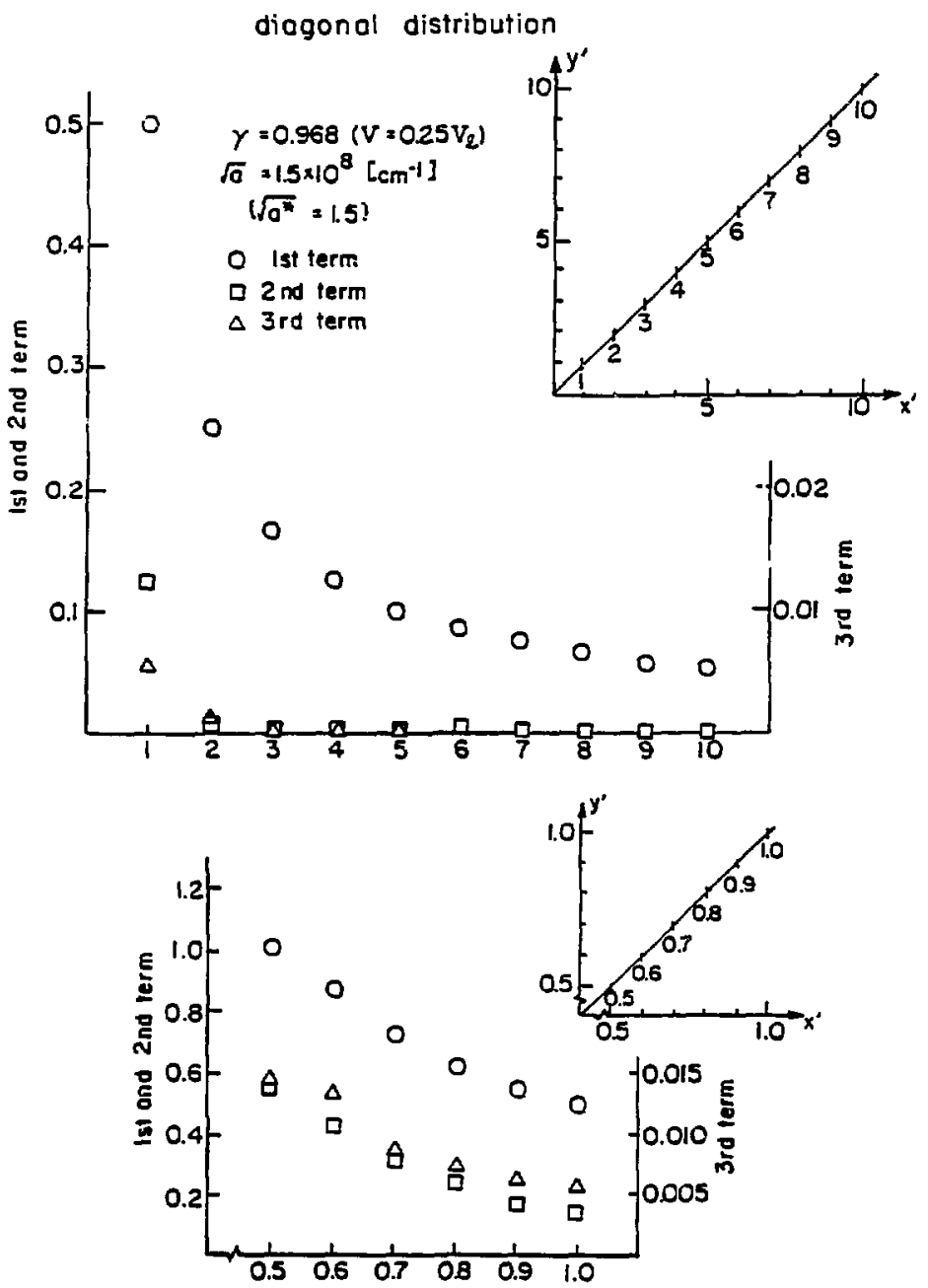

Figure z 
diagonal distribution

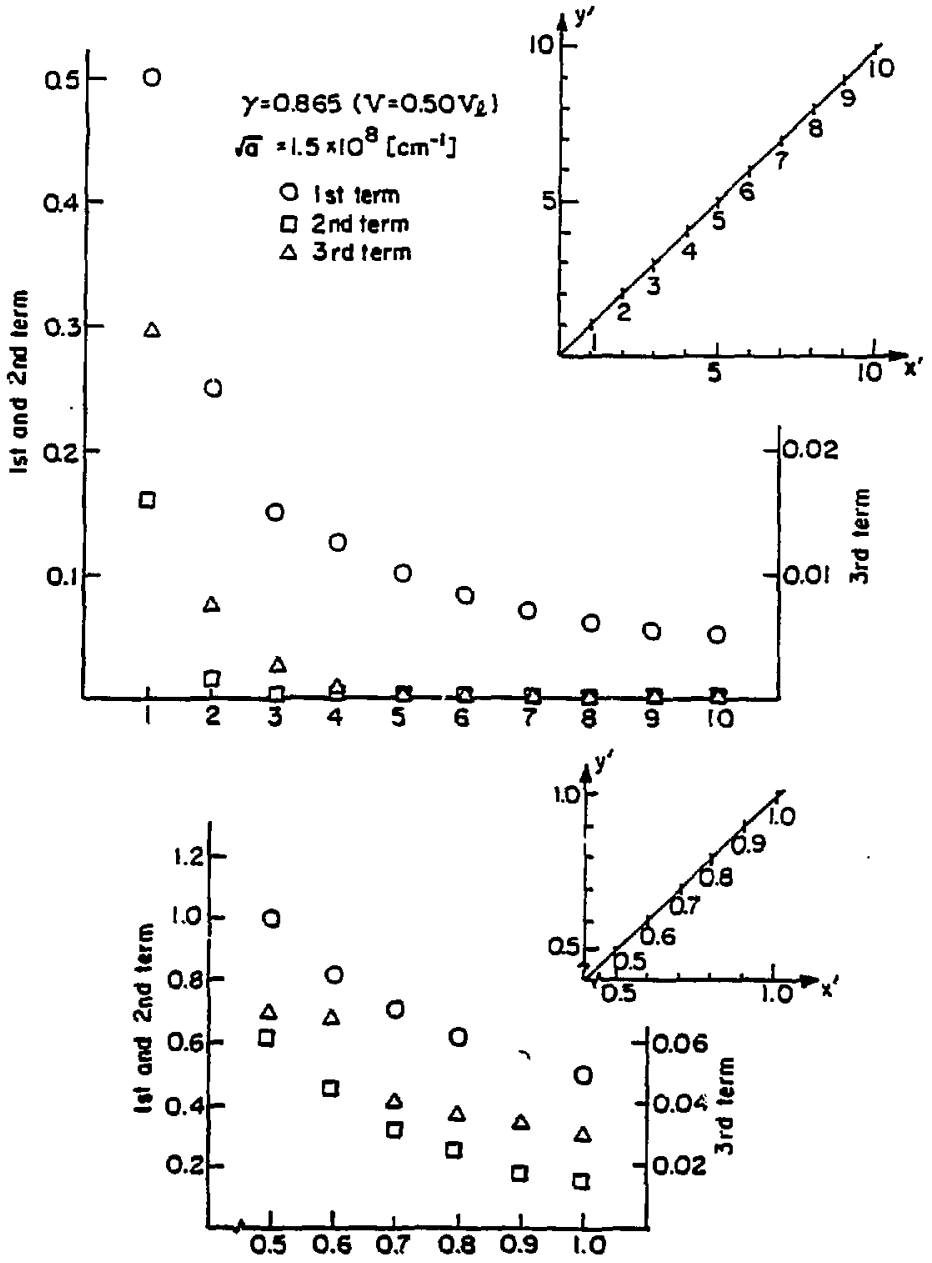



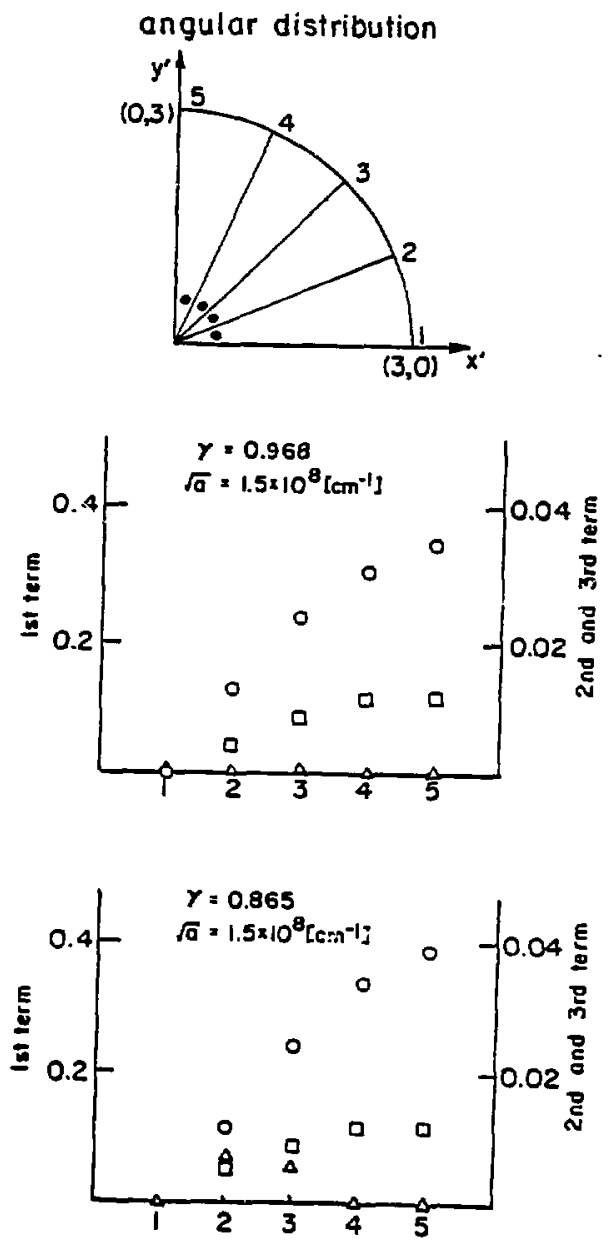


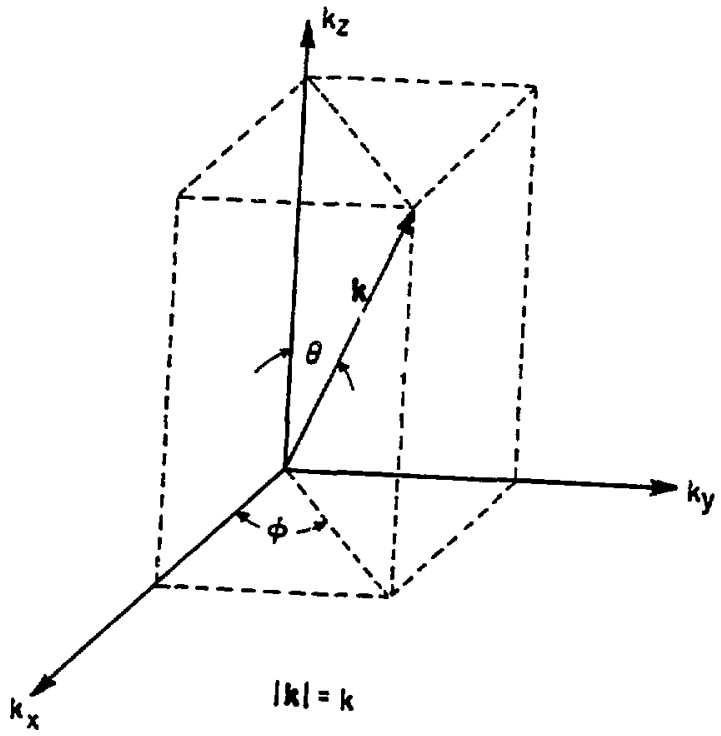

$x B L 9211-6809$

Figure 5 


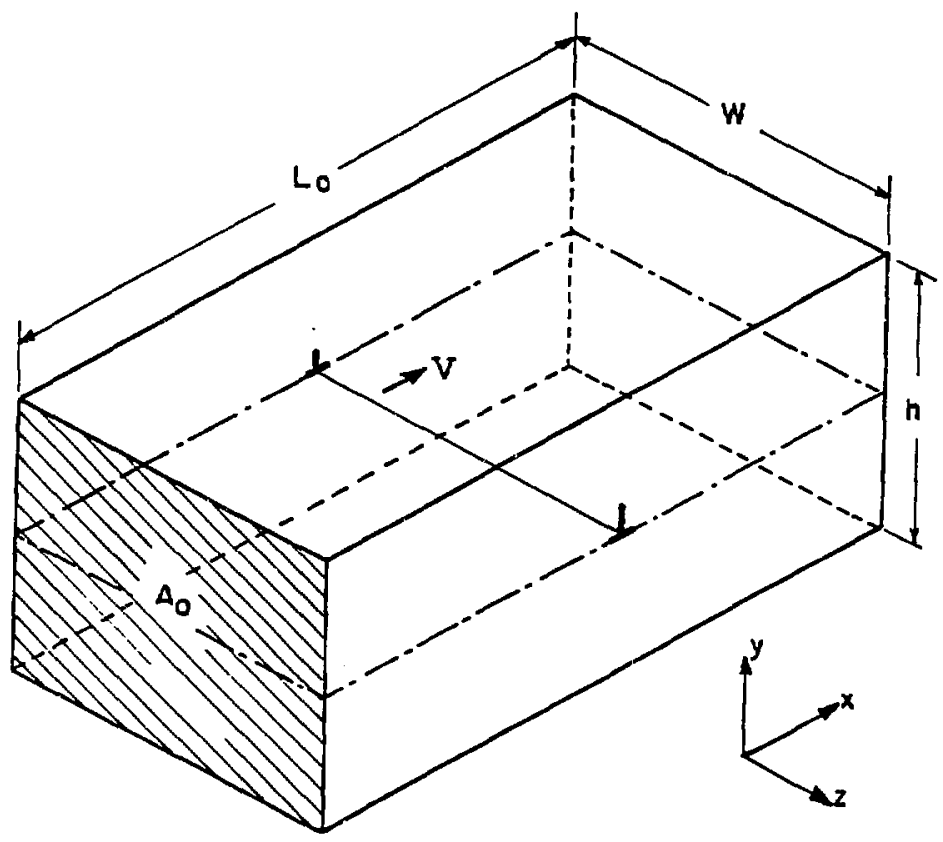

X8L8211-6810

Figure 6 


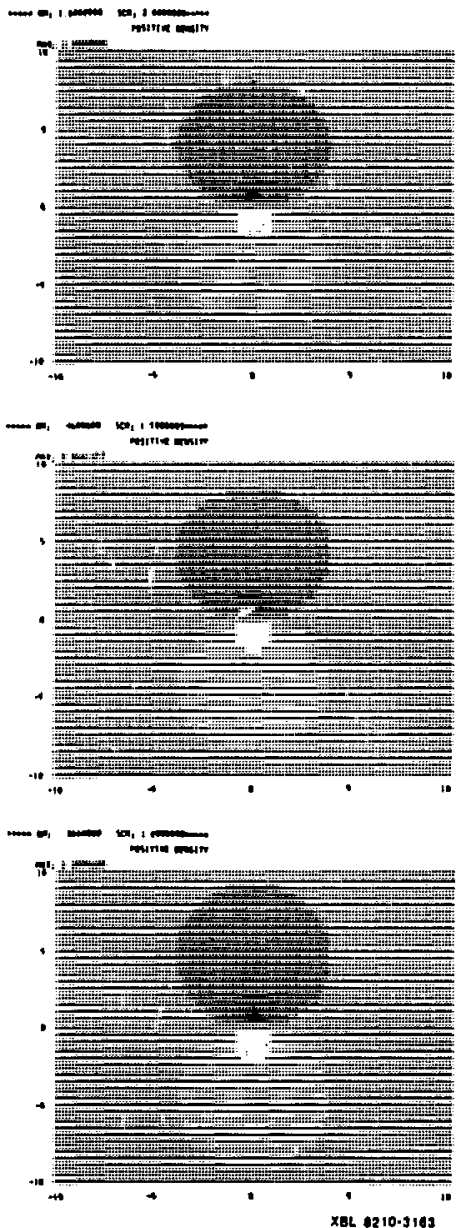

XBL 9210.3103 

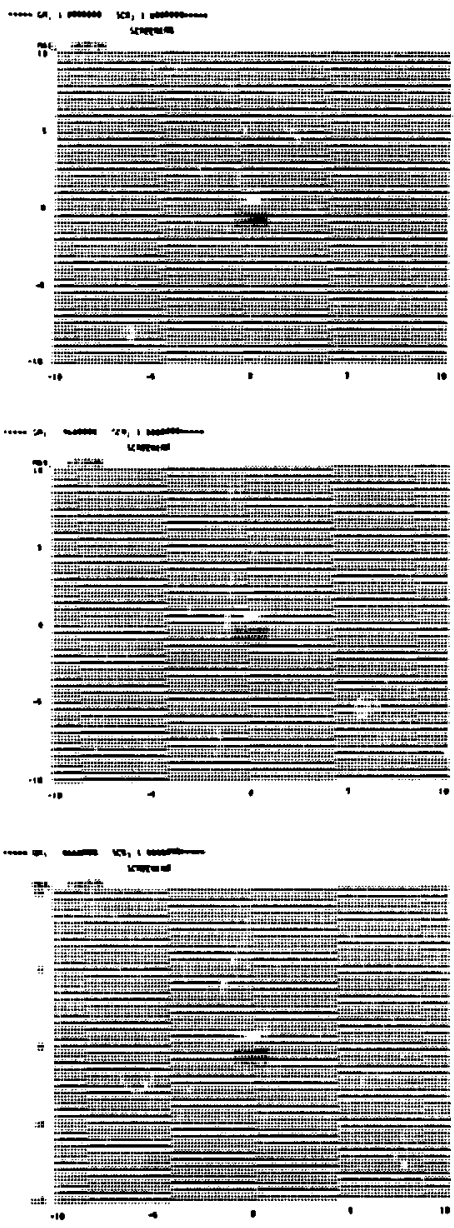

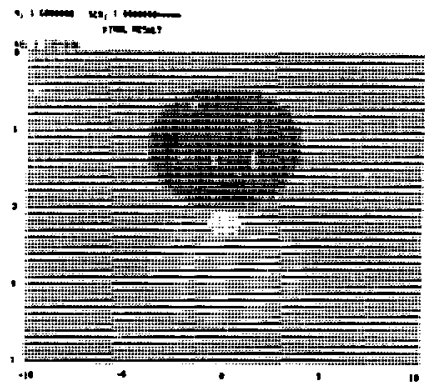

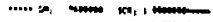

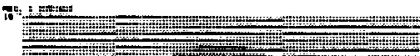

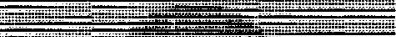

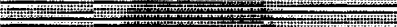
Win

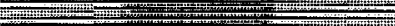

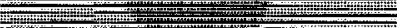

- 110 Fin VIn-

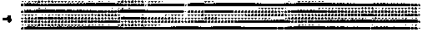

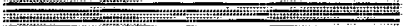
1

10

$-10$

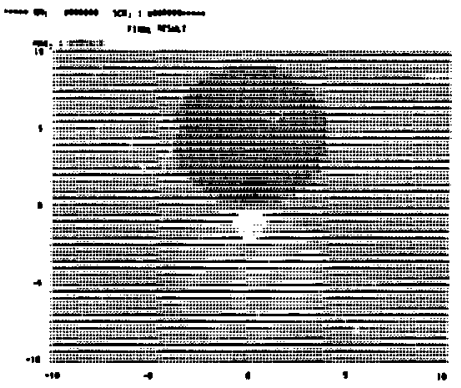

XEL 0210.3161

Figure 8 


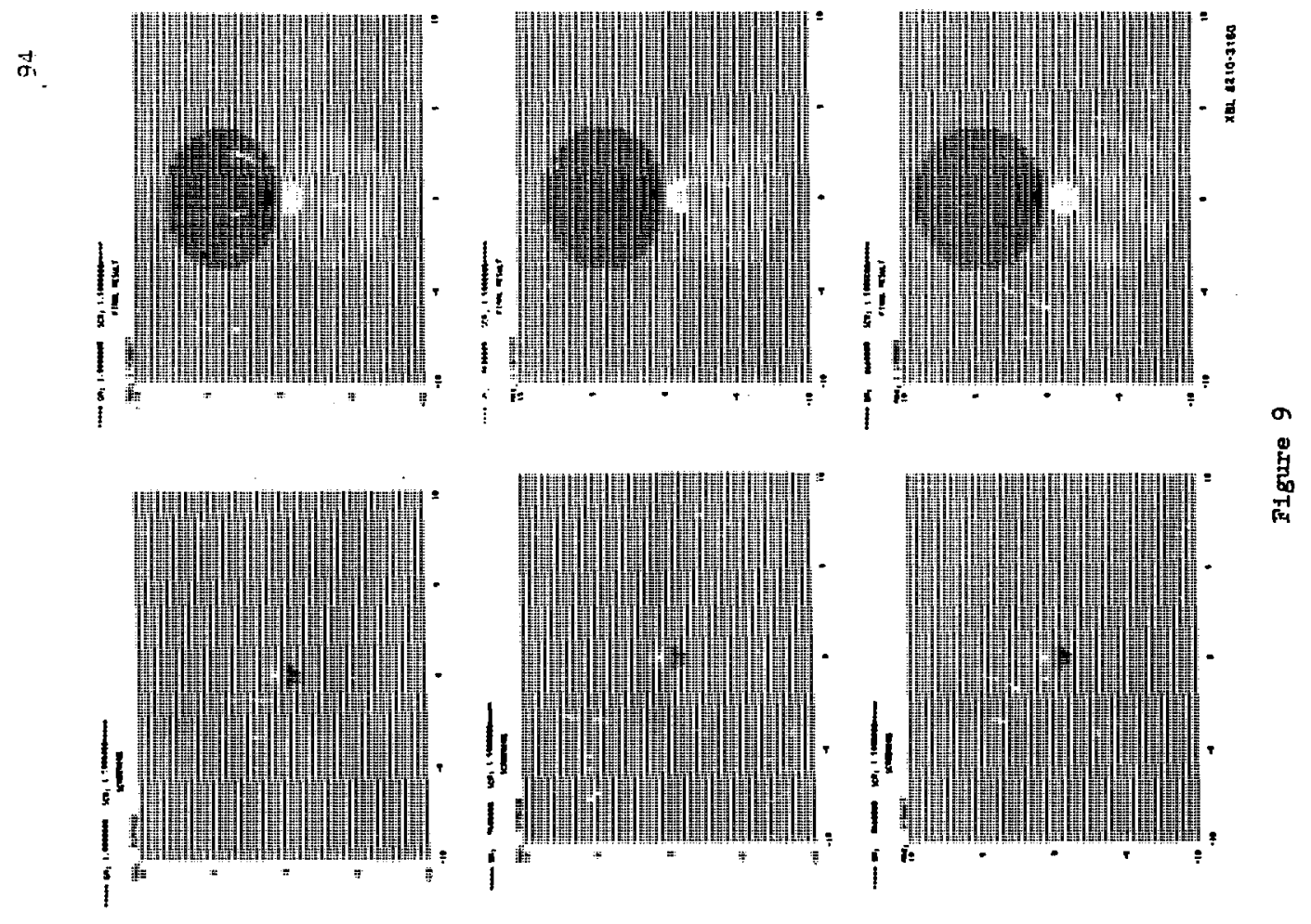




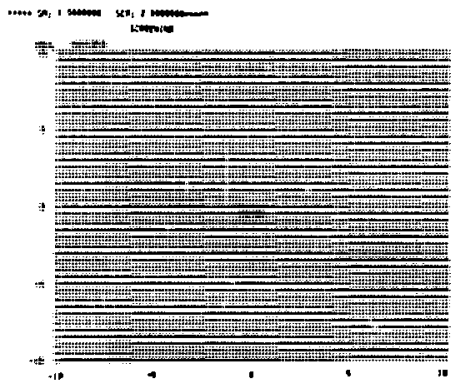

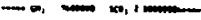

it:

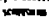

7)

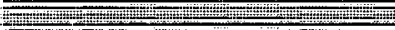

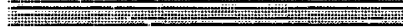

1 F F B B 7 +

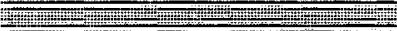

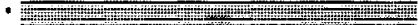
H

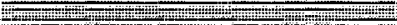

+4 At If -

.6 it

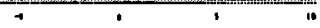

$\mathbf{A}^{\prime}, \mathrm{A}$

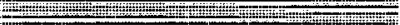
A

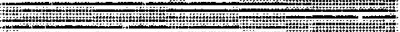
(

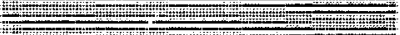
U-A O  证

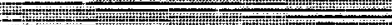

14 1 101010 (1)

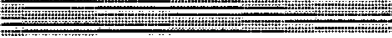

1

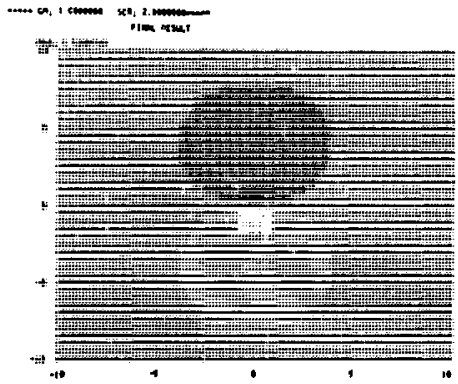

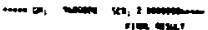

폴

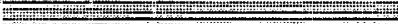

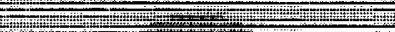

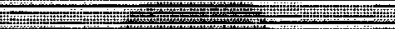

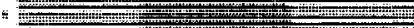
II

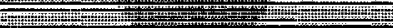

$x$ H

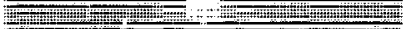

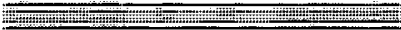

a

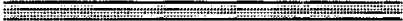
月10 10 $+1$

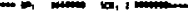

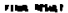

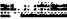

W

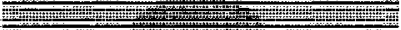
H

t.

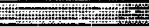

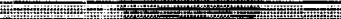

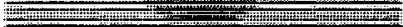
10

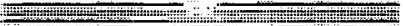

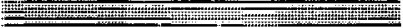

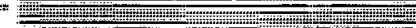
更

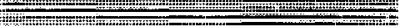
-

10

XBL 8210.1162 


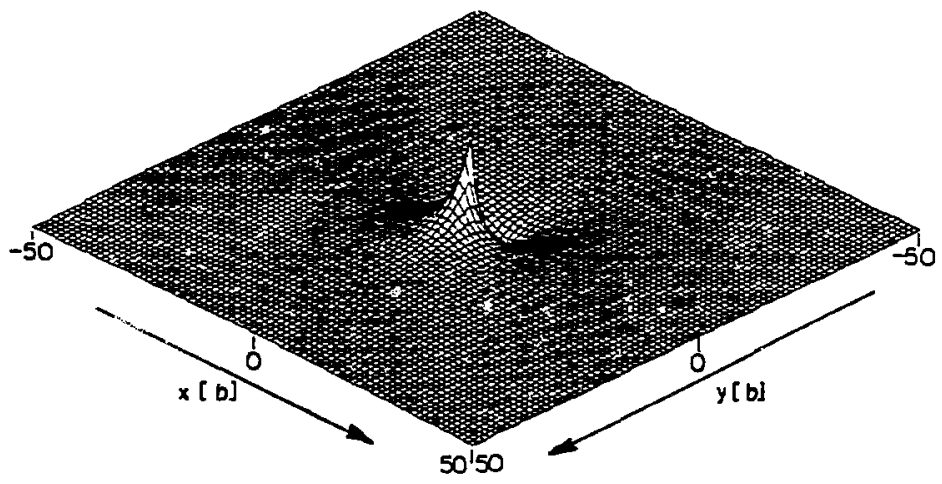

PERTURBATION POTENTIAL

$$
\begin{aligned}
y & =0.86 \\
\sqrt{\sigma} & =1.0 \times 10^{8}\left[\mathrm{~cm}^{-1}\right]
\end{aligned}
$$

X日L 8211-6819

Figure 41 


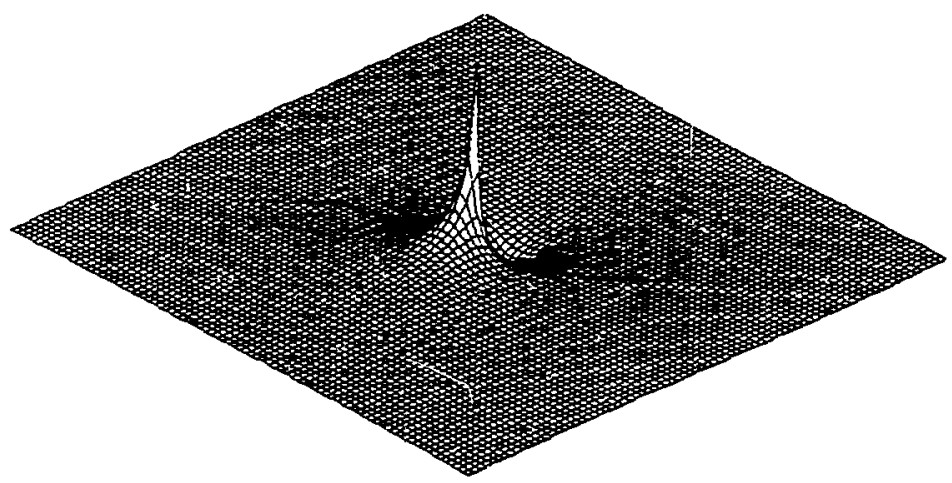

1 ST TERM

(positive chorge)

Figure 12 
98

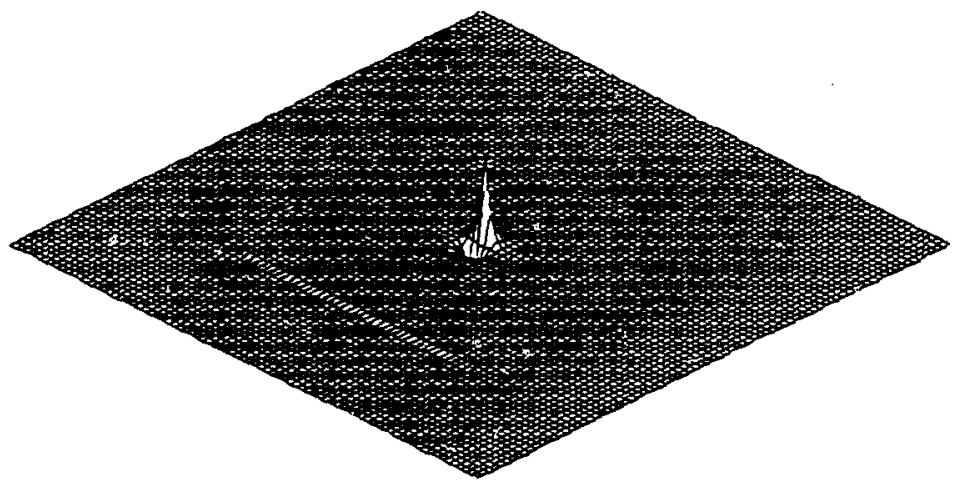

2ND TERM

(screening contribution)

XBL 82II-6821

Figure 13 


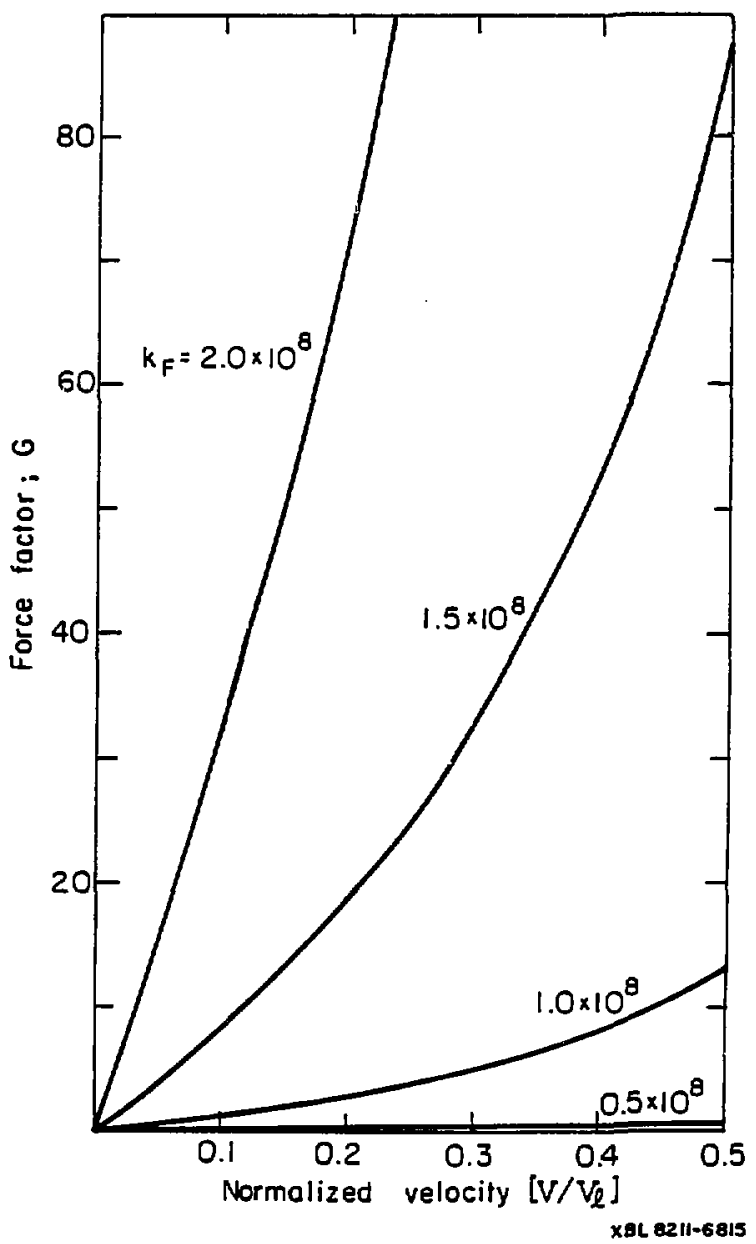

Figure 14 


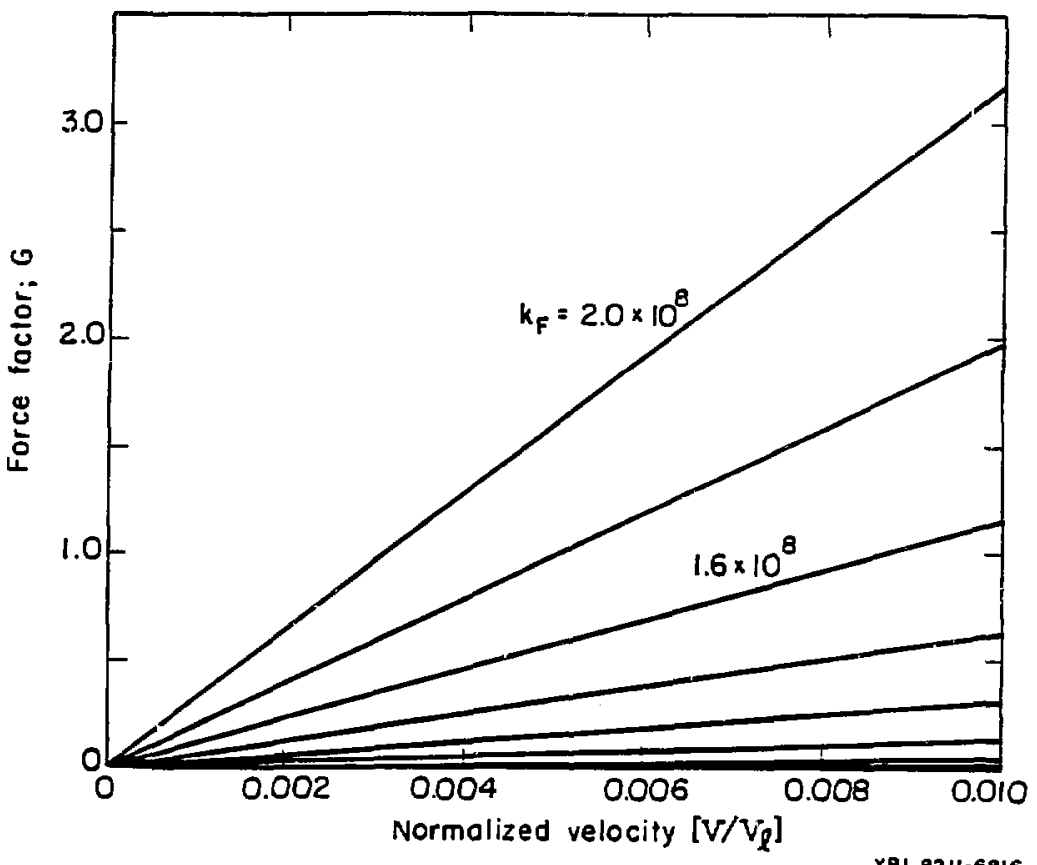

Figure 15 


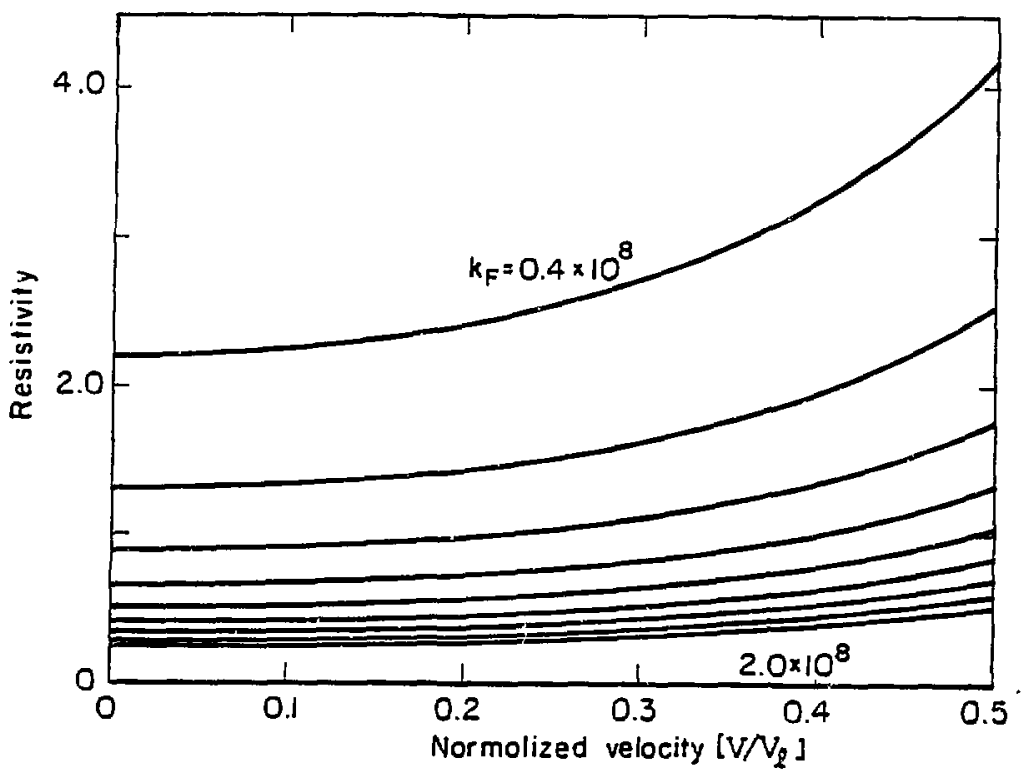

x8. $211-8017$

Figure 16 
geometrical distribution of magnetic indukition $v / v_{l}=0.3$
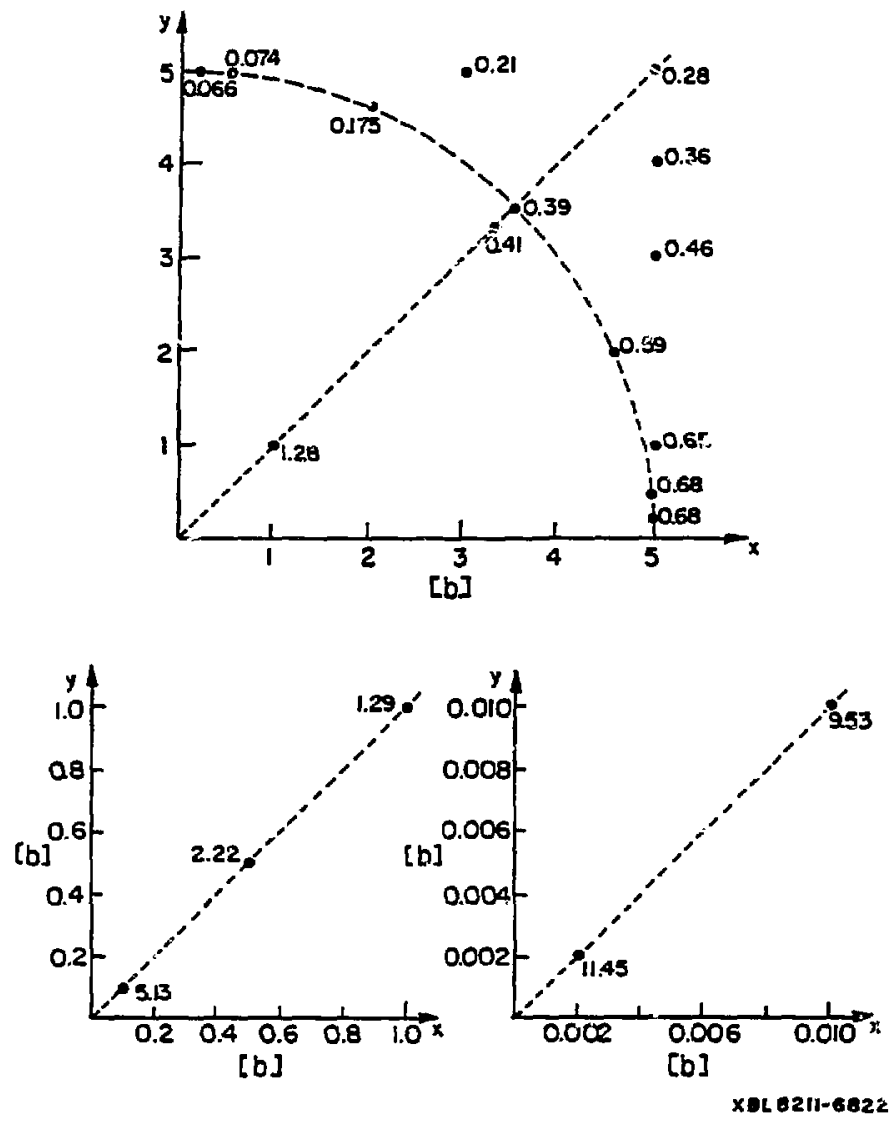


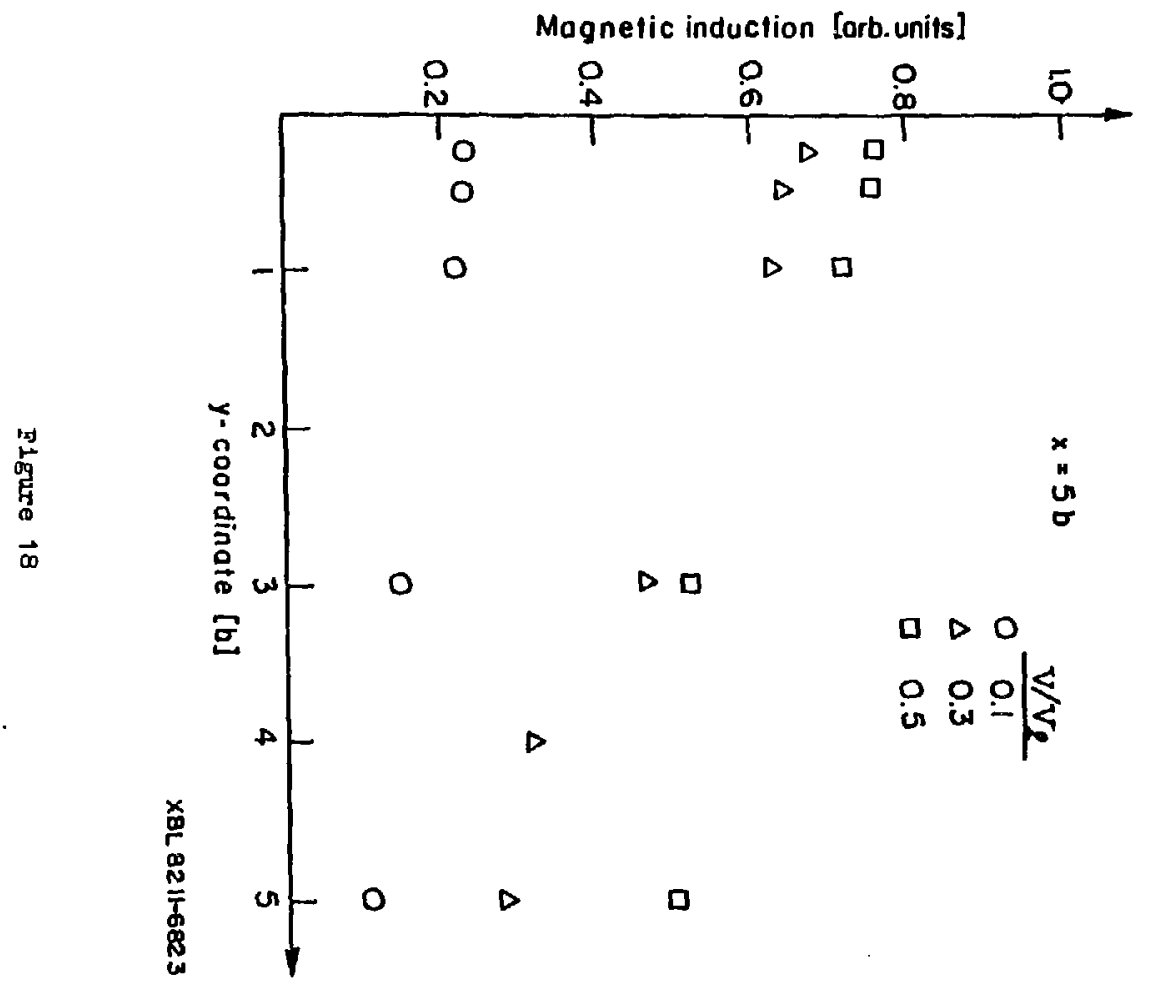




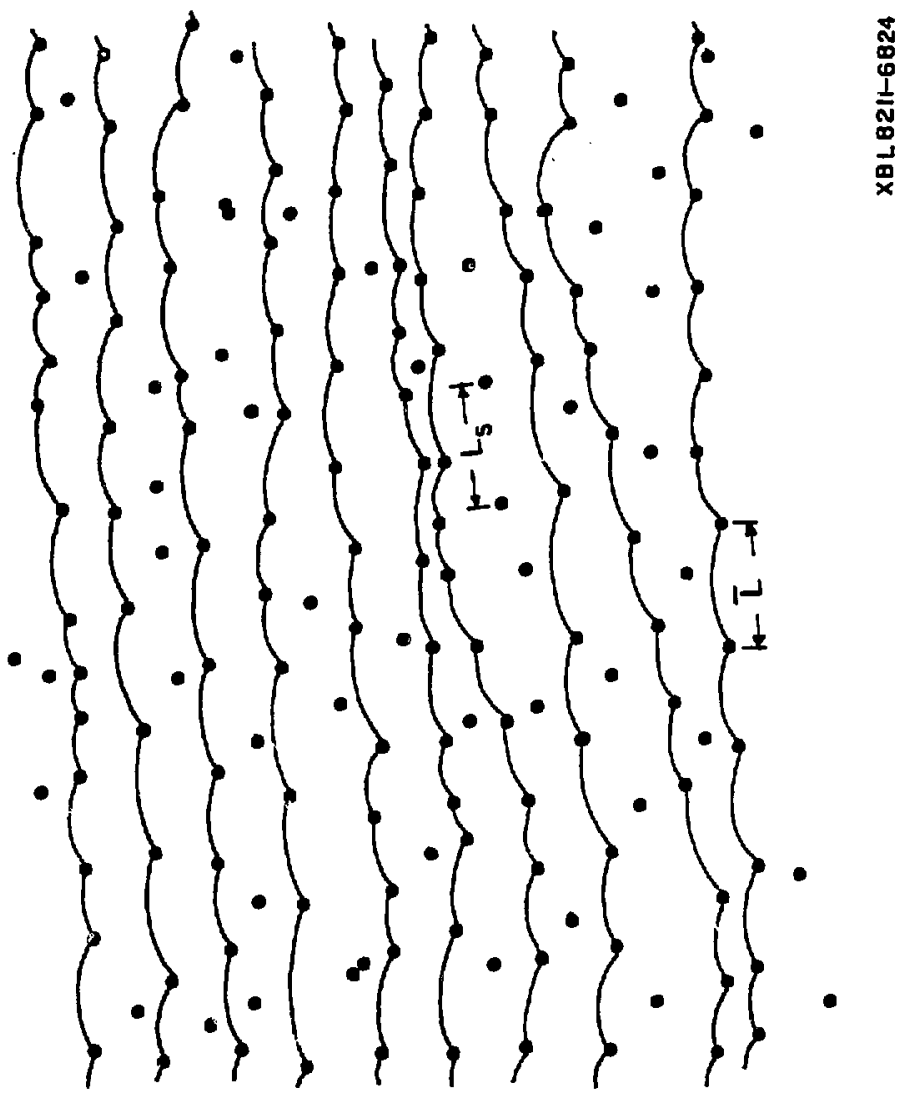

Figure 19 


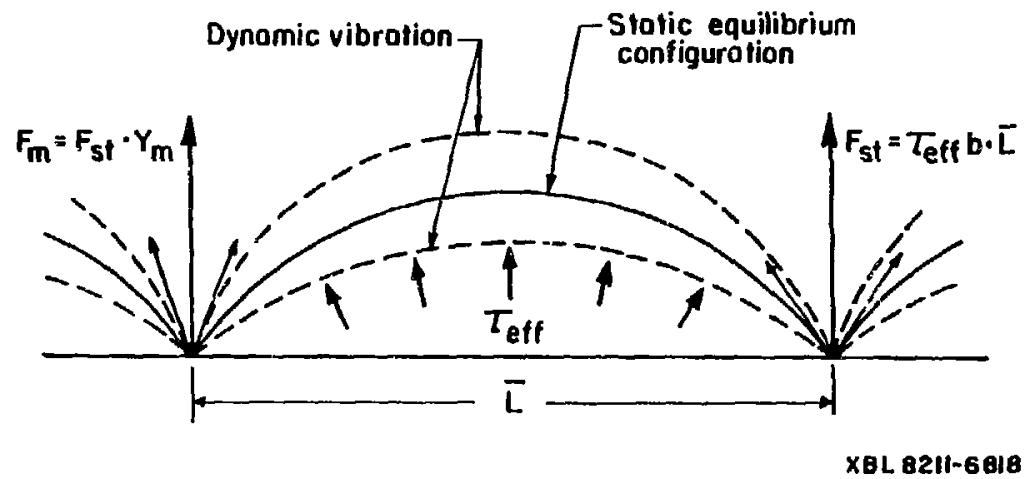




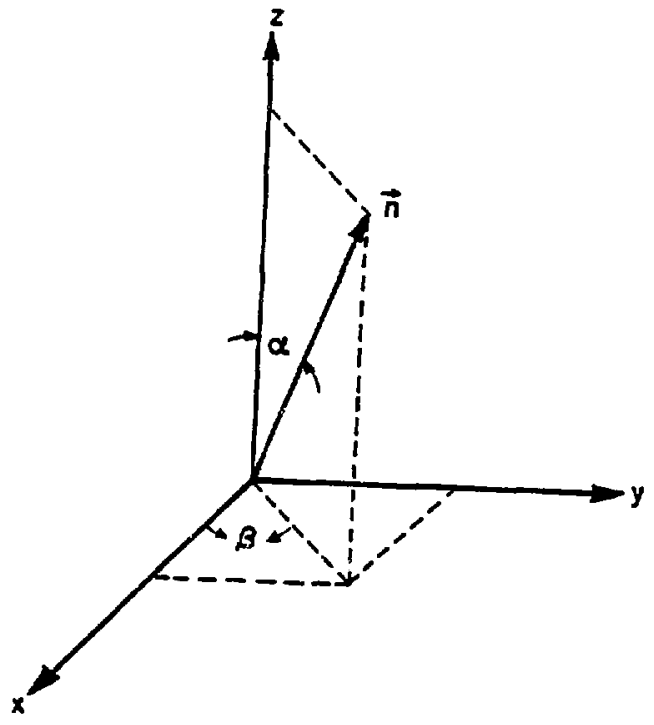

$x B L 8211-6813$

F1gure 21 


\section{LIST OF TABLES}

TABLE 1. Comparison of numerical and analytical values of resistivity

TABLF. 2. Lists of physical and material constants employed in this study

TABLE 3. Measured and caleulated electron damping constants 


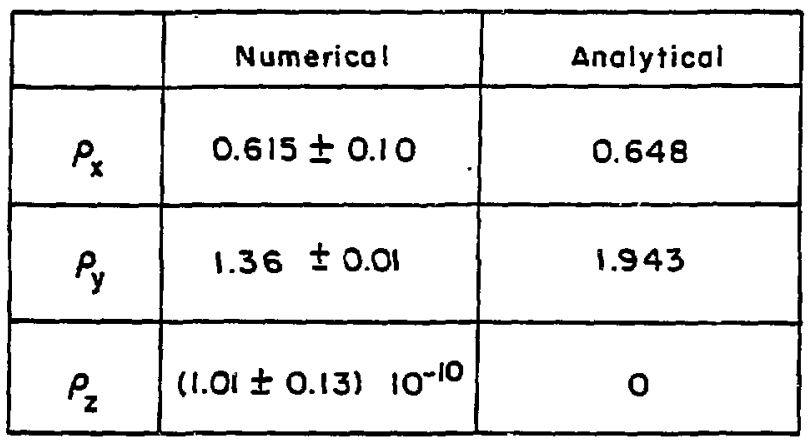

XBL 82II-6aI2

Table 1 


\begin{tabular}{|c|c|c|c|}
\hline & $\Delta !$ & $\mathrm{Cu}$ & Pb \\
\hline $\begin{array}{c}\mathbf{b} \\
\text { burgers } \\
\text { vector }\end{array}$ & $\begin{array}{l}2.86 \\
{[\AA]}\end{array}$ & 2.56 & 3.50 \\
\hline $\begin{array}{c}\text { KF } \\
\text { Fermi } \\
\text { vector }\end{array}$ & $\begin{array}{c}1.75 \\
\times 10^{8} \\
{\left[\mathrm{~cm}^{-1}\right]}\end{array}$ & $\begin{array}{r}1.36 \\
\times 10^{8}\end{array}$ & $\begin{array}{l}1.57 \\
\times 10^{8}\end{array}$ \\
\hline & 0.474 & 0.453 & 0.352 \\
\hline $\begin{array}{c}\text { m } \\
\text { mass of } \\
\text { electron }\end{array}$ & \multicolumn{3}{|c|}{$9.1 \times 10^{-28}[g]$} \\
\hline $\begin{array}{c}\text { h } \\
\text { Planck's } \\
\text { constant }\end{array}$ & \multicolumn{3}{|c|}{$1.1 \times 10^{-27}$ [erg-sec] } \\
\hline $\begin{array}{c}\text { e } \\
\text { elementory } \\
\text { chorge }\end{array}$ & \multicolumn{3}{|c|}{$4.8 \times 10^{-10}[\mathrm{esu}]$} \\
\hline
\end{tabular}

$X 8<8211-6814$

Table 2 


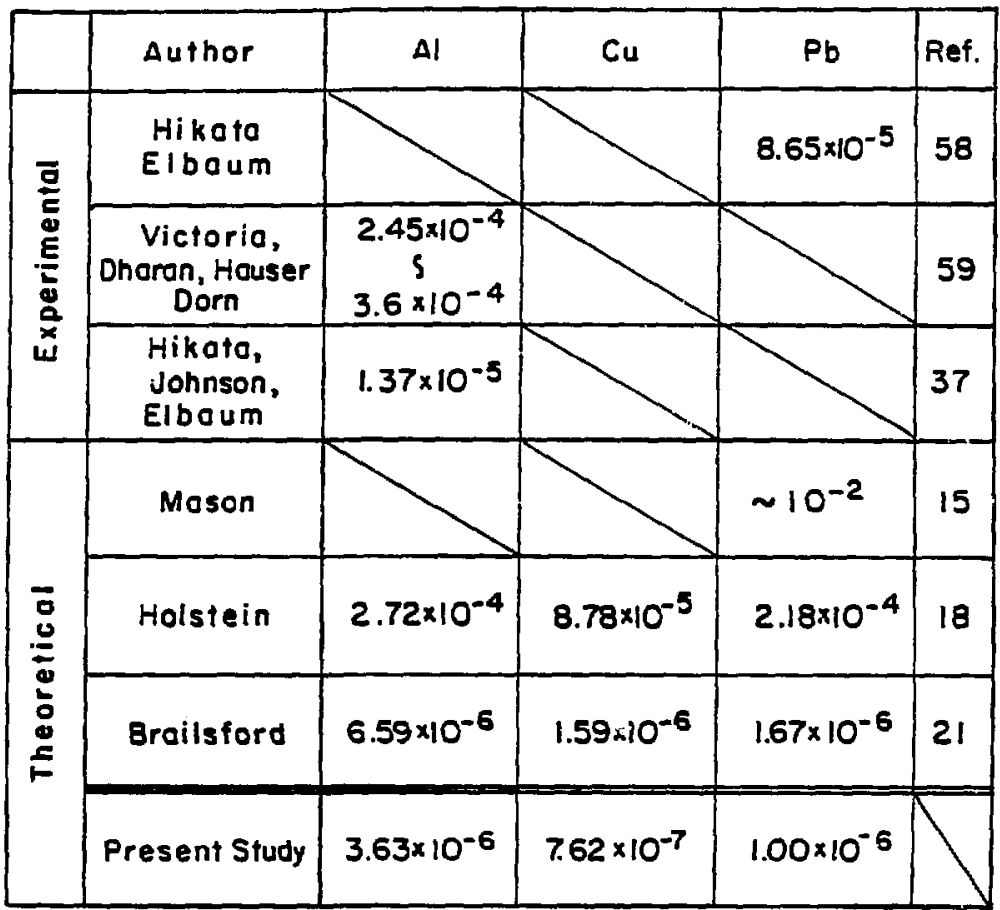

unit; dyn-sec/cm $\mathrm{cm}^{2}$

X BLB2II-6 al

Table 3 


\section{RAFFRENCES}

1. A. Eming and W. Rosenhain: Phil. Trans. Roy. Soc., At93 (L899) 353 "it is reported that 0 . Mugge had already suggested the existence of a dislocation in 1833.

2. H. Kojima and T. Suzuki: Phys. Rev. Letters, 21 (1968) 896

3. V. V. Pustovalov, V. I. Startsev, D. A. Didenko and V. S. Vermenko: Sov. Phys. Solid State, 11 (1969) 1119

4. K. Kamada and I. Yoshizawa: J. Phys. Soc. Japan, 31 (1971) 056 .

5. K. Marukawa: J. Phys. Soc. Japan, 22 (1967) 499

6. J. S. Koebler: Imperlections in Nearly Perfect Crystals (John wiley and Sons, Inc., New York, 1952), 197

7. T. P. Mason: J. Acoust. Soc. Amer., 32 (1960) 458

8. V. P. Soldator, V. I. Startser and T. I. Vainblat: phys. stat. sol. 37 (1970) 47

9. V. I. Startsev, V. P. Soldatov, V. D. Natsik and V. V. Abraimov: phys. stat. sol. (a) $59(1980) 377$

10. M. Suenaga and J. M. Galligan: Script. Met. 5 (1971) 63

11. J. M. Galligan, T. H. Lin and C. S. Pang: Phys. Rev. Letters, $3 B$ (1977) 405, J. M. Galligan and C. S. Pang: J. AppL. Phys. 60 (1979) 6253

12. F. IIda, T. Suzuki, E. Kramoto and S. Takeuchi: Tech. Rep. ISSP, Ser. A. (1978) No. 900

13. G. P. Hufman and N. Louat: Phys. Rev. Letters, 24 (1970) 1055

14. V. G. BAR'YAKHTAR, YE. I. DRUINSKTY and I. I. FAL'KO: Phys. Metals and Metallography 33 (1972) 1

15. Fr. P. Mason: Appl. Phys. Letters, 6 (1965) 111 
16. W. P. Mason: Phys. Rev., 143 (1966) 229

17. B. R. Tittmann and H. E. Bommel, Phys, Rev., 151 (1966) 178

18. T. Holstein (see APPENDXX to Ref. 17)

19. G. P. Hufman and N. P. Louat: Phys. Rev. Letters, 19 (1967) 510

20. G. P. Huffman and N. Louat: Phys. Rev., 176 (1968) 773

21. A. D. Brailstord: Phys. Rev., 186 (1969) 959

22. M. H. Cohen and M. J. Harrison: Phys. Rev., 117 (1960) 937

23. J. M. Ziman: Principles of the Theory of Solids (CAMBRIDGE UNIV. PRESS. 1972), chapt. 7.

24. J. D. Jackson: CLASSICAL ELECTRODYNAMICS (John Wiley and Sons, Inc., New York, (1963)), chapt. 11

25. K. Sumino: Bull. Japan Inst. Metals, 10 (1971) 693

26. A. H. Cottrell. S. C. Hunter and F. R. N. Nabarro: Phil. Mag., 44 (1953) 1064

27. Artken: Mathematical Method for Physicists (Academic Press), 760

28. J. Moore: PHYSICAL CHEMISTRY (Prentice-Hall. Inc., 1962), chapt. 14

29. J. M. Ziman (see section 7-1 to Ret. 23)

30. for example C. Kettel: Introduction to Solid State Physics (John Wiley and Sons, Inc. 1976) 5 th ed., chapt. 5

31. I. Stakgold: GREEN'S FUNCTIONS AND BOUNDARY VALUE PROBLEMS (John Wley and Sons, Inc.. New York, 1979), cbapt. 6

32. A. Matthiessen and C. Vogt: Ann Phys., Lpzg.. 122 (1864) 19

33. Ior example C. Kittel (see chapt. 6 to Ret. 30)

34. V. Vitek: Ptil. Mag., 21 (1970) 1275; Crystal Lattice Defects, 5 (1974) 1

35. J. Th. M. De Hosson: Int. J. Quantum Chemistry, 18 (1980) 575 
36. J. M. Ziman (see sectinn $4-5$ to Ref. 23)

37. A. Hikata, R. A. Johnson and C. Elbaum: Phys. Rev. Letters, 24 (1970) 2t5

38. Z. S. Basinski, J. S. Dugdale and A. Howie: Phil. Mag., 8 (1963) 1989

39. J. Bass: Adv. Phys., 91 (1972) 491

40. J. G. Rider and C. T. B. Foxon: Phil. Mag., 13 (1966) 289

41. for example C. Kittel (see chapt. 14 to Ref. 30)

42. J. J. Gilman and W. G. Johnston (Seitz and Turnbull ed., Solid State Physics 13. Academic Press 1962) 147, W. G. Jobrston: J. Appl. Phys., 33 (1962) 2716

43. H. Alexander and P. Haasen: Solid. State Physics, 22 (1968) 28

44. I. Yonezawa and K. Sumino: phys. stat. sol. (a), 50 (1978) 685

45. M. Suezawa, K. Sumino and I. Yonezawa: phys. stat. sol. (a). 51 (1979) 217

46. K. Sumino, S. Kodaka and K. Kojima: Mater. Sci. Eng., 13 (1974) 263

47. K. Nishioka and K. Obsaka: Phil. Mag. A. 37 (1978) 56i 48.. K. Sumino: Bull. Jap. Inst. Metals, 10 (1971) 761

49. J. J. Gillman: J. Appl. Phys., 39 (1968) 6c日6, E. Kuramoto, Y. Anno, K. Kitajima: Bull. Jap. Inst. Metals, 19 (1980) 348

50. J. Friedel: Dislocations (ADDISON-WESLEY PUBLISHING COMPANY, Inc., Mass., 1967)

51. A. V Granato: Phys. Rev. Letters, 27 (1971) 660; Phys. Rev.,B4 (1971) 2196 (see also Ret. 12)

52. R. D. Isaac and A. Y. Granato: (Proceed. 5th int. Conf. Strength of Intals and Alloys, Aachen, W. Germany (979) 490

53. S. Altintas: Ph.D Thesis, University of California, Berkely, 1978

54. K. Hanson and J. W. Morris, Jr.: J. Appl. Phys., 46 (1975) 2678 
55. I. Stakgold (see chapt. 2 to Ref. 31)

56. J. K. Mackenzie and E. H. Sondheimer: Phys. Rev. 77 (1950) 264

57. G. Campbell and R. M. Foster: Fourier Integrals( D. VAit NOETRAND COMPANY, INC.) No. 445

58. A. Hukrata and C. Elbaum: Phys. Rev. Letters, :B (1967) 750

59. M. P. Victoria, C. K. H. Dharan, F. E. Hauser and J. E. Dorn: J. Appl. Phys., 41 (1970) 674

60. T. Ninomiya: Bull. Jap. lnst. Metals, 12 (1973) 469

This report was done with support from the Department of Enerzy. Any conclusions or opinions expressed in this report represent solely those of the author(s) and not necessarily those of The Regents of the University of Californiz, the Lawrence Berkeley Laboralory of the Department of Energy.

Reference to a company or product name does not imply approval of tecommencation of the product by the University of California or the U.S. Department of Energy to the exclusion of others that may be suitable. 